\title{
One-dimensional Turbulence Models of Type I X-ray Bursts
}

\author{
A THESIS \\ SUBMITTED TO THE FACULTY OF THE GRADUATE SCHOOL \\ OF THE UNIVERSITY OF MINNESOTA \\ BY
}

Chen Hou

IN PARTIAL FULFILLMENT OF THE REQUIREMENTS FOR THE DEGREE OF

Doctor of Philosophy

Alexander Heger

January, 2016 
(C) Chen Hou 2016

ALL RIGHTS RESERVED 


\section{Acknowledgements}

The work that has been done to make this dissertation possible can be summarized in the following steps: learning the basic one-dimensional turbulence, or ODT, method, building a basic ODT model for astrophysical applications, adding pieces of physics into the basic model, validating each piece of physics by tests, analyzing results by comparison with a KEPLER model. For someone that knew a little about programming, this has been a long, challenging, and fruitful learning journey. There are many people that have earned my gratitude for their contribution to each step of the journey.

First, I want to thank Dr. Alan Kerstein for introducing the ODT method to me and for sending so many materials to help me choose the best way to start. This project would not have been kicked off without your patience and time in the discussions and feedback. Second, I want to thank Professor Alan Calder and Dr. Esteban Gonzalez for providing their data of Rayleigh-Taylor instabilities. I would like to thank Professor Frank Timmes for making his equation of state, nuclear networks, and opacity routines publicly available. They are valuable learning resources and have reduced plenty of my programming loads. I would also like to thank Dr. Laurens Keek for providing the original KEPLER model and for his discussion on the light curves and opacities. Additionally, I want to thank Dr. Duncan Galloway for providing the observational data of burster $4 \mathrm{U} 1820-30$.

I would like to acknowledge Professor Joseph Kapusta and especially Professor Yongzhong Qian for supporting me in my last semester of graduate school. It is one of the toughest periods of my life and your help and understanding is greatly appreciated.

I would also like to acknowledge my committee members Professor Joseph Kapusta, Professor Thomas Jones, and Professor Lawrence Rudnick for taking your time to read my dissertation and for providing feedback. 
Above all, I want to express my sincere gratitude to my advisor, Professor Alexander Heger, for his continuous guidance and help through the entire journey. Whether it is in the US, or in Australia, you have acted like a role model for me in science and in life. 


\section{Dedication}

To my parents who have supported me for this long even without understanding what I have been doing. 


\begin{abstract}
Type I X-ray bursts are caused by thermonuclear explosions occurring on the surface of an accreting neutron star in a binary star system. Observations and simulations of these phenomena are of great importance for understanding the fundamental properties of neutron stars and dense matter because the equation of state for cold dense matter can be constrained by the mass-radius relationship of neutron stars. During the bursts, turbulence plays a key role in mixing the fuels and driving the unstable nuclear burning process. This dissertation presents one dimensional models of photospheric radius expansion bursts with a new approach to simulate turbulent advection. Compared with the traditional mixing length theory, the one-dimensional turbulence (ODT) model represents turbulent motions by a sequence of maps that are generated according to a stochastic process. The light curves I obtained with the ODT models are in good agreement with those of the KEPLER model in which the mixing length theory and various diffusive processes are applied. The abundance comparison, however, indicates that the differences in turbulent regions and turbulent diffusivities result in more ${ }^{12} \mathrm{C}$ survival during the bursts in the ODT models, which can make a difference in the superbursts phenomena triggered by unstable carbon burning.
\end{abstract}




\section{Contents}

\begin{tabular}{ll}
\hline Acknowledgements & i
\end{tabular}

Dedication iii

Abstract iv

List of Tables vii

List of Figures viii

$1 \quad$ Type I X-ray Bursts 1

1.1 Historical background $\ldots \ldots \ldots \ldots$. . . . . . . . . . . . 1

1.2 Photospheric radius expansion bursts . . . . . . . . . . . . . . . . 3

1.3 Simulation techniques . . . . . . . . . . . . . . . . . 4

1.4 Outline of this dissertation . . . . . . . . . . . . . . 5

\begin{tabular}{lll}
\hline 2 & One-dimensional Turbulence Model & 7
\end{tabular}

2.1 Background . . . . . . . . . . . . . . . . . . . . . 7

2.2 Formulation . . . . . . . . . . . . . . . . . . . . 8

2.3 Application $\ldots \ldots \ldots \ldots \ldots \ldots \ldots$

\begin{tabular}{lll}
\hline 3 & Implementation & 14
\end{tabular}

3.1 Lagrangian grid . . . . . . . . . . . . . . . . . . . . . . . . 14

3.2 Flowchart . . . . . . . . . . . . . . . . . . . . . . . . . . . . . . . 18

3.3 Rayleigh-Taylor instability . . . . . . . . . . . . . . . 20

3.4 Equation of state . . . . . . . . . . . . . . . . . 27 


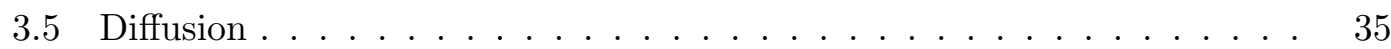

3.6 A simple burst model with three isotopes $\ldots \ldots \ldots \ldots$. . . . . . 42

4 Pure Helium Bursts Simulations Using ODT 449

4.1 Nuclear network . . . . . . . . . . . . . . . . . . . . . . . . . . . 49

4.2 Initial configuration $\ldots \ldots \ldots \ldots \ldots \ldots \ldots \ldots \ldots$

4.3 Formulation . . . . . . . . . . . . . . . . . . . . . . 53

4.4 Nuclear network comparison $\ldots \ldots \ldots \ldots \ldots \ldots \ldots$

4.5 Light curves . . . . . . . . . . . . . . . . . . . . 58

4.6 Turbulent motions and abundance $\ldots \ldots \ldots \ldots \ldots \ldots$

4.7 Conclusion $\ldots \ldots \ldots \ldots \ldots$

$\begin{array}{lll}5 & \text { Summary and Future Work } & 71\end{array}$

\begin{tabular}{|l|l|}
\hline Appendix A. Tests of Random Number Generator & 83
\end{tabular} 


\section{List of Tables}

1.1 Summary of early X-ray bursts observations . . . . . . . . . . . . . 2

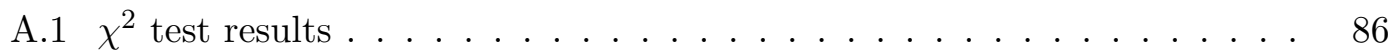

A.2 Correlation test results . . . . . . . . . . . . . . . . . . . 86 


\section{List of Figures}

$2.1 \quad$ Example of a triplet map on a linear profile $\ldots \ldots \ldots$. . . . . . . . 9

$\begin{array}{lll}3.1 & \text { Implementation of a triplet map on a simulation grid by permutation. . } & 16\end{array}$

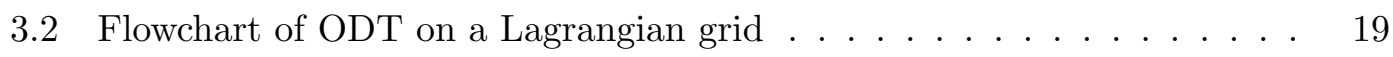

3.3 Initial density profile of RTI simulation . . . . . . . . . . . . . . 22

3.4 Density evolution of RTI . . . . . . . . . . . . . . . . . . . 25

3.5 Mixing thickness of RTI . . . . . . . . . . . . . . . . . . 26

$3.6 \quad$ Relative errors of $T$ given $(P, \rho)$ and iterations number . . . . . . . . . . 32

3.7 Relative errors of $\rho$ given $(P, T)$ and iterations number . . . . . . . . 33

3.8 Relative errors of $(\rho, T)$ given $(P, s)$ and iterations number. . . . . . . . 34

3.9 Relative errors of $(\rho, T)$ given $(P, h)$ and iterations number $\ldots . . . . \quad 35$

3.10 Comparison of two diffusion solvers . . . . . . . . . . . . . . . . . 41

3.11 Initial set-up of the 3-isotope model . . . . . . . . . . . . . . . . . . 43

3.12 Evolution of the nitrogen mass fraction profile. . . . . . . . . . . . . . 45

3.13 Evolution of the specific entropy profile $\ldots \ldots \ldots$. . . . . . . . . 46

3.14 Evolution of the velocity profile . . . . . . . . . . . . . . . 47

4.1 19-isotope nuclear network . . . . . . . . . . . . . . . . . . . 50

4.2 Initial set-up of the pure helium burst model $\ldots \ldots \ldots$. . . . . . . . . 52

$4.3 \quad$ Nuclear energy generation rates comparison without turbulent motion . 56

4.4 Abundance comparison at $55 \mathrm{~s}$ without eddy motion . . . . . . . . . 57

4.5 Abundance comparison at $65 \mathrm{~s}$ without eddy motion . . . . . . . . . 58

$4.6 \quad$ Light curves of the ODT models with different $C$ parameters . . . . . . $\quad 59$

4.7 Light curves comparison of the ODT model and the KEPLER model . . $\quad 60$

4.8 Mixing regions comparison . . . . . . . . . . . . . . . . . . . 63

4.9 Nuclear energy generation rates comparison with turbulent motion . . . 64 
4.10 Prominent abundance at $59 \mathrm{~s}$. . . . . . . . . . . . . . . 65

4.11 Prominent abundance at $59.8 \mathrm{~s} \ldots \ldots \ldots \ldots \ldots$. . . . . . . . . 65

4.12 Prominent abundance at $60 \mathrm{~s} \ldots \ldots \ldots$. . . . . . . . . . 66

4.13 Prominent abundance at $60.5 \mathrm{~s} \ldots \ldots \ldots$. . . . . . . . . 66

4.14 Prominent abundance at $62 \mathrm{~s} \ldots \ldots \ldots$. . . . . . . . . . 67

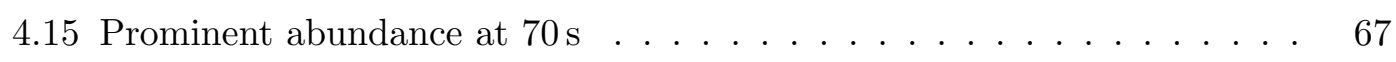

5.1 light curves comparison with observations . . . . . . . . . . . . 73

A.1 Uniformity test of one-sequence random numbers . . . . . . . . . . . . . 84

A.2 Uniformity test of two-sequence random numbers . . . . . . . . . . . . 85 


\section{Chapter 1}

\section{Type I X-ray Bursts}

In this chapter, I briefly review the historical background of Type I X-ray bursts research with a focus on photospheric radius expansion bursts phenomena and simulation techniques. There are several excellent review articles for a more comprehensive background introduction: Lewin \& Joss (1981), Lewin et al. (1993), and Strohmayer \& Bildsten (2006). In the end, I present the outline of this dissertation.

\subsection{Historical background}

In 1975, Grindlay \& Heise (1975) detected two "intense X-ray bursts" by the soft $\mathrm{X}$-ray experiment $(1-6 \mathrm{keV})$ and the hard X-ray experiment $(1-30 \mathrm{keV})$ on the Astronomical Netherlands Satellite (ANS; Gursky et al. 1975) and associated them with the X-ray source $3 \mathrm{U}$ 1820-30 in the globular cluster NGC 6624. Both events had short rise times $(\sim 1 \mathrm{~s})$ and decayed approximately exponentially at a time-scale of about $10 \mathrm{~s}$. The peak luminosities are about 20 and 30 times the background intensity, respectively. Independently, Belian et al. (1976) detected 20 "count-rate enhancements" $15 \sigma$ above background luminosity with detectors abroad the Vela satellites $5 \mathrm{~A}$ and $5 \mathrm{~B}(3-12 \mathrm{keV})$, 11 of which could be attributed to $10 \mathrm{X}$-ray flares from the same area in the constellation Norma. These two observations are usually considered as the discovery of X-ray bursts. Subsequently, more bursts were detected within half a year and they can be summarized along with aforementioned observations in Table 1.1. With the help of OSO-8 Satellite (2-24 keV; Swank et al. 1976), the number of burst sources cumulated 


\begin{tabular}{|c|c|c|c|}
\hline Reference and Time & Bursts & X-ray Source & Satellite \\
\hline Grindlay \& Heise $(\overline{1975)}$ & 2 & $3 \mathrm{U} 1820-30$ & $\overline{\mathrm{ANS}}$ \\
\hline Belian et al. $(\overline{1976})$ & 10 & $?$ & Vela 5 \\
\hline Clark et al. $(1976)$ & 10 & $3 \mathrm{U} 1820-30$ & SAS-3 \\
\hline Lewin $(1976)$ & 11 & MXB1743-293, MXB1742-297 & SAS-3 \\
\hline Lewin et al. (1976) & 29 & $\begin{array}{c}\text { MXB1743-293, MXB1742-297, } \\
\text { MXB1728-34 }\end{array}$ & SAS-3 \\
\hline Lewin et al. $(\overline{1976})$ & $\sim 10^{3} /$ day & MXB1730-335 (the Rapid Burster) & SAS-3 \\
\hline
\end{tabular}

Table 1.1: Summary of early X-ray bursts observations. From December 1975 to March 1976, about 8 burst sources were confirmed and 62 bursts (except those from the Rapid Burster) were observed. MXB stands for MIT X-Ray Burst Source. The question mark indicates that no known X-ray source could be associated to the observed bursts. The Rapid Burst does both Type I and Type II X-ray bursts, which explains its peculiarity on the large number of bursts.

to about 22 by the end of 1976 (Lewin 1977). Since 1995, the number of bursts observed has had a tremendous increase because of studies associated with several new satellites, i.e., RXTE (Galloway et al. 2008), BeppoSAX (e.g., Cornelisse et al. 2003), HETE II (e.g., Suzuki et al. 2007), and INTEGRAL (e.g., Chenevez et al. 2011). To date, X-ray bursts are probably the most frequent thermonuclear explosions in the universe with a day count of about 11 thousands (in 't Zand 2011).

Several theoretical models were proposed to explain X-ray bursts but were ruled out quickly. Even before the discovery, Hansen \& van Horn (1975) published the first theoretical paper about thermonuclear burning on the surface of neutron stars. Later this idea was developed by Woosley \& Taam (1976) and Maraschi \& Cavaliere (1977), which became supported by more and more observations, e.g., Swank et al. (1977) and Hoffman et al. (1977) measured the blackbody radii of sources and found them comparable to the size of a neutron star. But the Rapid Burster could not be explained by thermonuclear explosions because an otherwise present high flux of persistent X-ray emission was not observed. Hoffman et al. (1978) resolved this puzzle by discovering that the Rapid Burster emitted two different kinds of bursts: one was due to thermonuclear flashes and the other was due to the accretion instabilities. They also introduced the classification of Type I and Type II X-ray bursts for these two kinds, respectively. For 
the remainder of this dissertation, only Type I X-ray bursts (hereafter called XRBs or simply bursts) are considered. After decades of development, theory describes an XRB as a thin-shell thermonuclear instability that occurs in a binary system after a neutron star accretes enough fuels through Roche Lobe overflow from a low-mass companion (less than $\left.\sim 1 M_{\odot}\right)$. When the accumulated fuels are heated and compressed to a certain threshold, a series of thermonuclear reactions happen and drive an outburst with its energy released mainly as X-ray photons. The mass donors are typically old Population II stars or degenerate helium or carbon/oxygen white dwarfs (Rappaport et al. 1982). Depends on the composition of the fuels, the nuclear burning enters different regimes to trigger an XRB (see Strohmayer \& Bildsten 2006 for a detailed discussion).

\subsection{Photospheric radius expansion bursts}

A regular XRB profile shows a short rise time $(\sim 1 \mathrm{~s})$ and an exponential decay time ( $\sim 10$ s). Long bursts that last up to 15 minutes have been observed (e.g., Tawara et al. 1984b). These events usually start with a precursor which is a brief increase of intensity lasting for a few seconds. After the intensity returns to the persistent flux level for several seconds, the main event starts with a gradual increase in the hardness of the blackbody spectrum until the maximum blackbody temperature has reached. Then the main event decays in a similar way to what is observed in a regular XRB. Theoretical work (Tawara et al. 1984a; Lewin et al. 1984) proposed that energy released during these bursts was large enough to cause an expansion of the photosphere. Because the luminosity is nearly constant early on in the burst, an increase in the photospheric radius leads to a decrease in the effective temperature. If the expansion is large enough, the effective temperature can be shifted entirely below the X-ray band. Thus a gap between the precursor and the main event is observed. These bursts are called photospheric radius expansion (PRE) bursts. Subsequent work indicates that, the luminosity during the expansion stays within a small percentage of the Eddington limit, and the excess luminosity is transformed efficiently into kinetic and potential energy of the outflow (Paczynski \& Proszynski 1986, Joss \& Melia 1987; Shaposhnikov \& Titarchuk 2004). When the burst is not that powerful, the burst profile may show a double-peak feature instead of a precursor as only part of the flux is shifted. 
PRE bursts are of great importance in the following two aspects. First, they can be considered as distance indicators. Since the peak luminosity is very close to the Eddington limit (about $3.8 \times 10^{38} \mathrm{erg} / \mathrm{s}$ for hydrogen-poor atmosphere), this limit can provide a "standard candle" to within about $15 \%$ according to the test of a set of globular clusters with independently known distances (Kuulkers et al. 2003). Second, PRE bursts, in principle, provide a way to infer the mass-radius relationship of neutron stars (see Lewin et al. 1993 for a detailed discussion). Here I only outline the main idea. After the expansion, the photosphere starts to contract and the ending point of this process is called "touchdown" at which the photosphere return to its original radius. The Eddington luminosity is given by

$$
L=(4 \pi c G M / \kappa)\left(1-2 G M / R c^{2}\right)^{-1 / 2},
$$

where $M$ is the mass of the star, $R$ is the radius measured by a local observer, and $\kappa$ is the opacity of the photosphere. The gravitational redshift is included as the second bracket above. The relation of the luminosity to the effective temperature $T_{\text {eff }}$ is

$$
L=4 \pi R^{2} \sigma T_{\mathrm{eff}}^{4},
$$

where $\sigma$ is the Stefan-Boltzmann constant. So $T_{\text {eff }}$ at "touchdown" can be expressed in terms of $M, R$, and $\kappa$. A mass-radius relationship can be determined if $T_{\text {eff }}$ is measured and an atmosphere model is applied. Usually, $T_{\text {eff }}$ is obtained from the observed color temperature. The importance of a mass-radius relationship lies in the fact that it constrains the equation of state of neutron star matter (Lattimer \& Prakash 2001), which is a fundamental problem of cold dense matter.

\subsection{Simulation techniques}

Because of the complexity of XRBs problems, e.g., large nuclear networks, diffusion processes, and the equation of state, numerical simulation functions as an important tool for theoretical studies from the very beginning. In fact, numerical models based on a combination of multiple integrators appeared in the first theoretical paper of XRBs (Hansen \& van Horn 1975) about 10 months before the phenomena were even observed.

After decades of development, there are three simulation approaches in general: one-zone models, multi-zone models or one-dimensional models, and multi-dimensional 
models. As the name indicates, one-zone models use only one zone for numerical calculations with models retaining the fundamental physics that is necessary to yield qualitative, and even quantitative results similar to full-scale computations (e.g., Paczynski 1983). They usually require less computing time and programming effort. But some one-zone simulation uses the computing power to focus on particular aspects of the problem in detail. For example, Schatz et al. (2001) explored the rapid proton ( $r p)$ capture process of hydrogen burning during an XRB and found the end point of this process was a closed SnSbTe cycle. One-dimensional models are probably the most prevalent choice because of the advantage as a compromise between one-zone and multi-dimensional models. Many of these models have successfully reproduced lots of observable features of XRBs such as peak luminosities, rise times, durations, and recurrence times (e.g., Woosley \& Weaver 1984; Taam et al. 1993; Woosley et al. 2004; Heger et al. 2007). But the model inherited assumptions such as uniformly distributed accreting fuels are highly unrealistic. Besides, the convection motion are usually simulated by the stellar mixing length theory (Böhm-Vitense 1958) or through various diffusive processes (Heger et al. 2000), which don't always agree well with multi-dimensional simulations Arnett et al. 2009). Ideally, multi-dimensional models, especially 3D models, are the closest to reality, but the requirement of computational resources makes it rather restrictive and less practical. So far, there is limited number of published work with this technique, over half of which assumes an idea gas law in incompressible regime without thermal diffusion (Fryxell et al. 2000; Zingale et al. 2001; Spitkovsky et al. 2002). More recently, A low-Mach number approximation method is developed to improve the input physics and applied to simulate convective burning at the base of an accreted layer of a burst (Lin et al. 2006). By using MAESTRO, both the large-scale heating and small-scale compressibility effects are captured in simulating the convection before a pure helium burst (Malone et al. 2011). Later this technique is improved and applied to the simulation of a mixed hydrogen/helium burst (Malone et al. 2014).

\subsection{Outline of this dissertation}

Since this dissertation focuses on the numerical hydrodynamic simulation of XRBs, I first introduce the basic idea of the simulation approach (one-dimensional turbulence 
model) we chose in this dissertation in Chapter 2. In Chapter 3, I describe all the adaptations we made to prepare this model for XRBs simulations including the choice of coordinate, the stellar equation of state, and the diffusion processes. Several tests and applications are discussed to verify these adaptations. After the code is described, I present the results of a pure helium burst simulation that demonstrates PRE features and show the comparisons with those of a KEPLER model in Chapter 4. Finally, I summarize what has been accomplished and discuss the future work to improve the results. 


\section{Chapter 2}

\section{One-dimensional Turbulence Model}

In this chapter, I start with a brief introduction to the background of the onedimensional turbulence (ODT) model. Then I explain the formulation in detail because of the unique features in its formulation that distinguishes ODT from most of the other turbulence simulation methods, and the intactness these features maintain to the adaptations that are discussed extensively in Chapter 3 . Finally, I discuss the applications of ODT models.

\section{$2.1 \quad$ Background}

The fact that molecular mixing in turbulence is sensitive to both of the large-scale and small-scale fluid motions makes it an important role to test turbulence models. The effort of simulating molecular mixing dates back at least to the coalescence-dispersion model (Curl 1963). Only the direct numerical simulation (DNS), however, can solve the Navier-Stokes equation and molecular diffusion equations with sufficient resolution but at the expense of computing time and power. Kerstein (1991) sorted the other approaches into two categories: computational models intended to be flexible tools, and conceptual models addressing specific issues. These models either simplify the microscopic mixing by assuming that the mixing is implemented instantaneously within an appropriate length-scale (e.g., Kolmogorov scale), or sacrifice the mechanistic distinction 
to some extent between molecular and convective processes. Under these circumstances, the linear-eddy model, or LEM, highlights its advantages in the following aspects. First, all the relevant length-scales are resolved. The fluid motion is considered as a sequence of instantaneous measure-preserving maps that are called triplet maps, whereas the microscopic motion is implemented by molecular diffusion directly. Second, the mapping events are implemented according to a Poisson process. The mechanistic distinction that is partly lost in the previous models can now be evaluated by a wide variety of mixing-field statistics. More importantly, it preserves the successful feature of the twoparticle-dispersion model. Although LEM is conceived as a computationally efficient way to model the mixing of passive scalars, the flow properties are specified empirically by assigning parameters governing the Poisson process. ODT was developed based on LEM in the sense that it kept the two aforementioned features. But ODT pushed the stochastic model forward to minimal empiricism by allowing the scalar fields to self-consistently determine the rate of turbulent mixing in the model.

\subsection{Formulation}

Following the two special features as mentioned in Section 2.1. ODT has two major assumptions. First, there is only one kind of eddies in the turbulent system and it can be represented by an instantaneous map. Second, these maps form a sequence of events governed by a stochastic Poisson process with its frequency determined by the current stability of the flow.

The triplet map is chosen as the standard measure-preserving map in most ODT and LEM applications, although there is no strict constraint on it. A quintuplet map was used in some computation to determine the sensitivity of the results to the choice of maps (Kerstein 1991). In this dissertation, only triplet map is discussed and used because it is the simplest in its class. Figure 2.1 shows the effect of a triplet map on an initially linear scalar field (e.g., concentration). The eddy region $(1 \leq y \leq 4$, where $y$ marks the 1D simulation domain) is divided into three segments. The first and the third segments are formulated by compressing the original eddy region by a factor of three along $y$-direction. And they are attached by the second segment that is a copy of either segment but with a reversed gradient. It is obvious that the map doesn't 


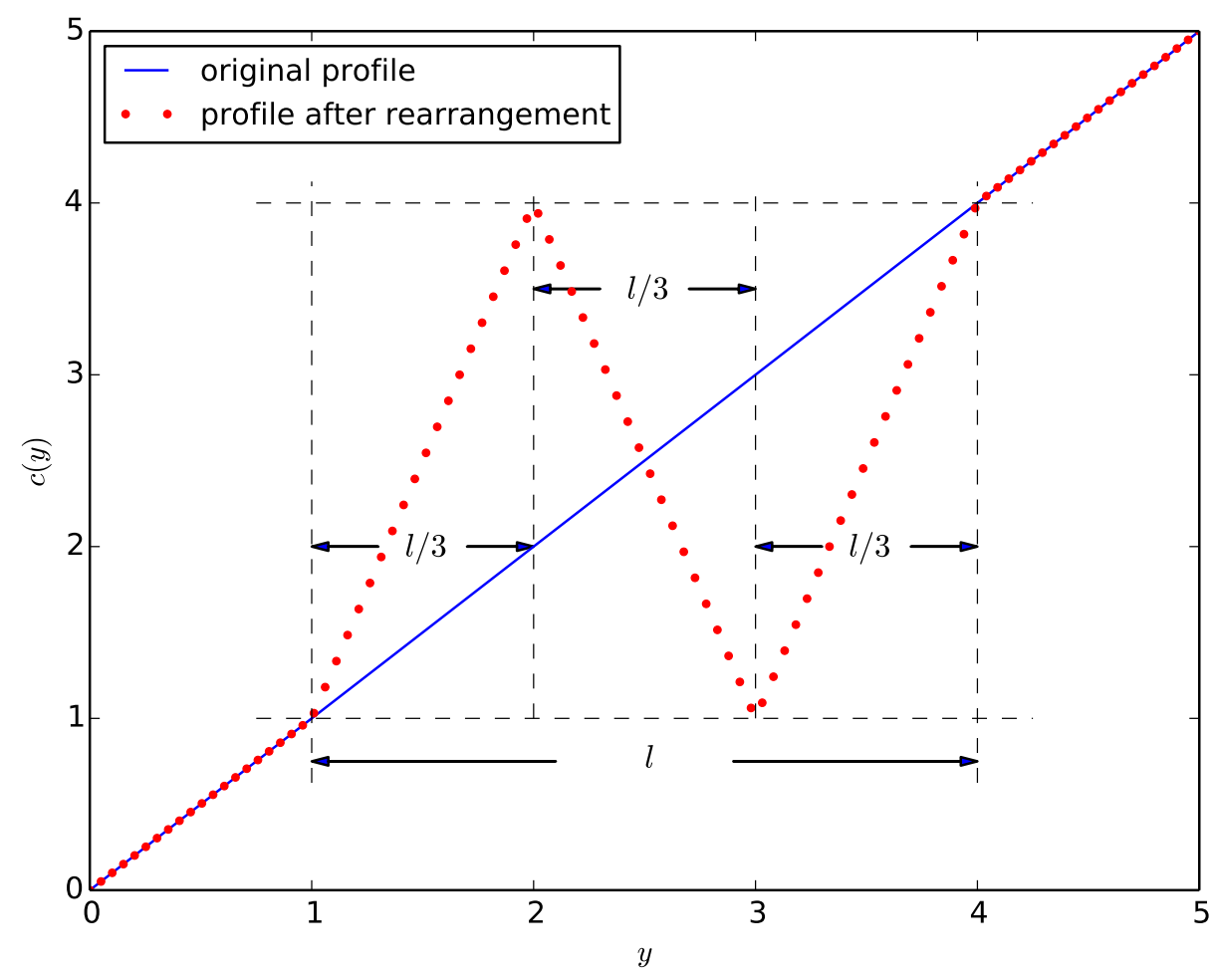

Figure 2.1: Example of a triplet map on a linear profile. The eddy region $l$ is divided equally into three segments. The first and third segments are obtained by increasing the original gradient by a factor of three. The second segment is the reversed copy of the first one.

introduce any spatial discontinuity to the scalar field and the choice of instantaneous implementation can be considered as a compromise to guarantee the continuity. After the rearrangement, the strain intensity increases and the strain length-scale decreases, which mimics the rotational and compressive effect of a real eddy. Here the three segments have the same length in $y$, which gives uniform strain intensity multiplication and length-scale reduction. There are situations when non-uniform maps are considered but with additional empirical parameters. Again, these situations are not considered in this dissertation. During the rearrangement, the non-local displacement imitates the largescale fluid motion and keeps the physical integrity of the fluid elements. Operationally, 
a triplet map can be expressed as

$$
f(y)= \begin{cases}y_{0}+3\left(y-y_{0}\right) & \text { if } y_{0} \leq y \leq y_{0}+\frac{1}{3} l \\ y_{0}+2 l-3\left(y-y_{0}\right) & \text { if } y_{0}+\frac{1}{3} l \leq y \leq y_{0}+\frac{2}{3} l \\ y_{0}+3\left(y-y_{0}\right)-2 l & \text { if } y_{0}+\frac{2}{3} l \leq y \leq y_{0}+l \\ y & \text { otherwise }\end{cases}
$$

where $l$ is the eddy length, and $y_{0}$ is one end of the eddy region. Consider a velocity field $u(y)$ during this transformation

$$
u(y) \rightarrow u[f(y)]+c K(y)
$$

where the $c K(y)$ term is introduced in order to conserve total energy. The kernel is defined as $K(y)=y-f(y)$. The momentum will be automatically conserved for an incompressible fluid on an Eulerian grid, since

$$
\int_{y_{0}}^{y_{0}+l} K(y) \mathrm{d} y=0 .
$$

It is helpful to derive the following identity first.

$$
\begin{aligned}
& \int_{y_{0}}^{y_{0}+l} K^{2}(y) \mathrm{d} y=\int_{y_{0}}^{y_{0}+l}[y-f(y)]^{2} \mathrm{~d} y \\
= & \int_{y_{0}}^{y_{0}+l / 3}\left(2 y-2 y_{0}\right)^{2} \mathrm{~d} y+\int_{y_{0}+l / 3}^{y_{0}+2 l / 3}\left(4 y-4 y_{0}-2 l\right)^{2} \mathrm{~d} y+\int_{y_{0}+2 l / 3}^{y_{0}+l}\left(2 y-2 y_{0}-2 l\right)^{2} \mathrm{~d} y \\
= & \left.\left(\frac{4}{3} y^{3}-4 y_{0} y^{2}+4 y_{0}^{2} y\right)\right|_{y_{0}} ^{y_{0}+l / 3}+\left.4\left[\frac{4}{3} y^{3}-2\left(2 y_{0}+l\right) y^{2}+\left(2 y_{0}+l\right)^{2} y\right]\right|_{y_{0}+l / 3} ^{y_{0}+2 l / 3} \\
& +\left.4\left[\frac{1}{3} y^{3}-\left(y_{0}+l\right) y^{2}+\left(y_{0}+l\right)^{2} y\right]\right|_{y_{0}+2 l / 3} ^{y_{0}+l} \\
= & \left(\frac{4}{81} l^{3}+\frac{8}{9} y_{0} l^{2}\right)+\frac{4}{81} l^{3}+\left(-\frac{8}{9} y_{0} l^{2}+\frac{4}{81} l^{3}\right) \\
= & \frac{4}{27} l^{3}
\end{aligned}
$$

For the convenience of later discussion in this chapter, $y$ is chosen as the spatial coordinate, and potential energy is added to kinetic energy to solve the energy conservation 
equation

$$
\begin{aligned}
\frac{1}{2} \rho_{0} \int_{y_{0}}^{y_{0}+l} u^{2}(y) \mathrm{d} y+g \int_{y_{0}}^{y_{0}+l} \rho(y) y \mathrm{~d} y= & \frac{1}{2} \rho_{0} \int_{y_{0}}^{y_{0}+l}(u[f(y)]+c K(y))^{2} \mathrm{~d} y \\
& +g \int_{y_{0}}^{y_{0}+l} \rho[f(y)] y \mathrm{~d} y .
\end{aligned}
$$

Here Boussinesq approximation is applied and $\rho_{0}$ is the reference density. Combining identities (2.3) and (2.4), and $u(y)=u[f(y)]$, it yields a quadratic equation for $c$

$$
\frac{2}{27} l^{3} c^{2}+u_{K} l^{2} c+\frac{g}{\rho_{0}} \Delta E_{g}=0
$$

with

$$
u_{K} \equiv \frac{1}{l^{2}} \int_{y_{0}}^{y_{0}+l} u[f(y)] K(y) \mathrm{d} y
$$

and

$$
\Delta E_{g} \equiv \int_{y_{0}}^{y_{0}+l}(\rho[f(y)]-\rho(y)) y \mathrm{~d} y .
$$

The solution of the above equation is

$$
c=\frac{27}{4 l}\left[-u_{K}+\operatorname{sgn}\left(u_{K}\right) \sqrt{u_{K}^{2}-\frac{8 g}{27 \rho_{0} l} \Delta E_{g}}\right] .
$$

The turbulent energy term inside the square root reflects the change of total energy between the configurations before and after the map. In other words, it can be used to measure the stability of the flow in the eddy region. If $c$ is imaginary, this implies that the region examined is stable in terms of energy and that there is not enough energy to drive a turnover. The calculation above only included one dimensional velocity profile for the purpose of simplification. In fact, ODT is capable of capturing some features of 3D fields (Kerstein 1999), in which one needs to consider that the turbulent energy may redistributed in an anisotropic way (Ashurst \& Kerstein 2005). In this dissertation, a simple isotropic option is adopted because the properties of interest are not sensitive on this matter.

Similar to LEM, ODT generates a sequence of mapping events according to a Poisson process. Although a triplet map is implemented instantaneously, it should occur at a time scale $\tau\left(l, y_{0}, t\right)$ that is approximate to the real eddy turnover time. One main distinction in ODT is that $\tau$ is treated as a local, instantaneous time scale rather than 
a mean time scale as in LEM. There are different approaches to estimate the eddy time scale based on dimensional analysis. Kerstein (1999) started from the local strain and considered it in the Fourier picture. The others (Kerstein \& Dreeben 2000; Ashurst \& Kerstein 2005; Kerstein \& Wunsch 2006; Gonzalez-Juez et al. 2011) usually associate it directly to the eddy driving energy. From the previous calculation, the relation can be expressed in the following way

$$
\left(\frac{l}{\tau}\right)^{2} \sim u_{K}^{2}-\frac{8 g}{27 \rho_{0} l} \Delta E_{g}-Z \frac{\nu^{2}}{l^{2}}
$$

The first two terms match the turbulent energy term in the square root of Equation (2.9). If both terms are positive and the whole term increases, the driving of the eddy becomes stronger and the required time for a region of size $l$ to mix convectively becomes shorter. On the contrary, if both terms decrease and the whole term reaches negative value, the eddy turnover time increases till no eddy occurs. The last term is added to represent the viscous damping effect with the first free parameter $Z$ serving as a local Reynolds number to suppress small eddy events with time scales longer than the viscous time scale $l^{2} / \nu$. At last, the eddy rate distribution can be expressed as

$$
\lambda\left(l, y_{0}, t\right) \equiv \frac{C}{l^{2} \tau\left(l, y_{0}, t\right)}=\frac{C}{l^{3}} \sqrt{u_{K}^{2}-\frac{8 g}{27 \rho_{0} l} \Delta E_{g}-Z \frac{\nu^{2}}{l^{2}}},
$$

where $C$ is the second free parameter that controls the overall eddy rate. Strictly speaking, $\lambda\left(l, y_{0}, t\right) \mathrm{d} l$ is the frequency of events in the size range $[l, l+\mathrm{d} l]$ per unit length at time $t$ and location $y_{0}$. The instantaneous frequency is capable of collecting feedback directly from the current flow status, but requires costly reconstruction at each time step. To resolve this nonstationary Poisson Process, ODT applies a thinning algorithm (Kelton \& Law 2000) to reduce the computing load. Briefly speaking, thinning algorithm chooses a $\lambda^{\prime}$ always bigger than the actual rate so that the system advances at the smallest time step possible to avoid missing any feedback from the flow. At each time step, an eddy trial is carried out at a certain location and $\lambda$ is calculated according to Equation 2.11). By comparing $\frac{\lambda^{\prime}}{\lambda}$ to a random number $U(0,1)$, the eddy trial is decided to be accepted $\left(\frac{\lambda^{\prime}}{\lambda} \geq U(0,1)\right)$ or rejected $\left(\frac{\lambda^{\prime}}{\lambda}<U(0,1)\right)$. In this way, the time step becomes smaller when the flow is turbulent in order to capture all the information of the flow, and the time step becomes bigger when the flow is stable in order to save eddy sampling time. 


\subsection{Application}

Due to the limit of spatial dimensionality, ODT manages to capture as many flow properties as possible by generally dividing the problems into two categories in its application (Kerstein 1999): temporal flows (T-flows) and spatially developing flows (Sflows). T-flows are represented by a time evolution of the transverse profile of streamwise velocity and other scalar profiles. In this category, ODT has been applied to the Couette flow, channel flow, pipe flow, double diffusive convection (Gonzalez-Juez et al. 2011), and reactive Rayleigh-Taylor turbulent mixing (Gonzalez-Juez et al. 2013). S-flows are different in that the realizations are parameterized by two spatial dimensions (streamwise coordinate $x$ and vertical coordinate $y$ ) instead of $y$ and time $t$ as in T-flows. The velocity along $y$-direction is solved by the combination of the momentum diffusion equation and the continuity equation. In this category, ODT has been applied to buoyancy-driven flows such as the Rayleigh convection, penetrative convection in the atmospheric-boundary-layer problem, and meter-scale buoyant turbulent flames (Ricks et al. 2010). 


\section{Chapter 3}

\section{Implementation}

In this chapter, I describe the development of my version of ODT. For better adaptation to the astrophysical problems of my interest, a Lagrangian grid is chosen over an Eulerian grid. The advantages and disadvantages of this choice are discussed in detail below. A flowchart is presented to explain how ODT processes on the newly chosen grid. After adding in a large-eddy-suppression mechanism, ODT is tested with the Rayleigh-Taylor instability problem and the results are compared with those of 3D direct numerical simulations (DNS). The Helmholtz equation of state is adopted and modified for different cases in the stellar application, each of which is tested on both accuracy and efficiency. Two diffusion solvers included in ODT are discussed and compared by diffusing a step-function profile on an isolated grid. Finally, a simple XRB model with 3-isotope burning is presented to test all the building pieces included so far.

\subsection{Lagrangian grid}

In previous applications, ODT has been applied primarily on an Eulerian grid because of convention and specific problems. For similar reasons, we decide to apply ODT on a Lagrangian grid for our applications. In detail, the advantages of a Lagrangian grid are listed as follows.

First, no numerical diffusion is introduced to a simulation on a Lagrangian grid. The transformation from Lagrangian coordinates to Eulerian coordinates can be expressed 
as a material derivative

$$
\frac{\mathrm{D}}{\mathrm{d} t}=\frac{\partial}{\partial t}+\mathbf{u} \cdot \nabla
$$

The non-linear advection term on the right side introduces numerical diffusion on an Eulerian grid, which sometimes causes especially difficult problems in simulations like modeling thermonuclear burning fronts (LeVeque et al. 1998). It is of great advantage that it is not present on the left side of the equation that corresponds to a Lagrangian grid. Although this advantage of the Lagrangian formulation breaks down in some multi-dimensional simulations in which the comoving grid becomes very distorted, it is not a concern in our one-dimensional turbulence model. On an Eulerian grid, ODT usually treats problems as T-flows or S-flows (Section 2.3). For T flows, only the term $\frac{\partial}{\partial t}$ is considered for time evolution. For S-flows, the other term, $\mathbf{u} \cdot \nabla$, is solely included in the calculation for two-dimensional spatial evolution. Because there is only time derivative on a Lagrangian grid, it falls into T-flows category.

Second, when the flow is compressible, ODT in the Eulerian formulation has to resolve the following two issues (Ashurst \& Kerstein 2005). The mass flux induced by molecular diffusion yields an auxiliary variable that is called mass-average velocity. It can be eliminated but requires adopting a molecular transport model such as Fickian transport. Another issue is that the momentum conservation is no longer automatically maintained by property $(2.3)$ simply because the density can no longer be pulled out of integration of equation

$$
\int \rho(y) u(y) \mathrm{d} y=\int \rho[f(y)] u[f(y)] \mathrm{d} y .
$$

An extra term has to be included in the velocity transformation to enforce momentum conservation, which also causes complexity to the energy conservation. These two issues become trivial in the Lagrangian formulation. The mass-average velocity is caused by advection and is included in the advection term of the Navier-Stokes equations. This factor is not present on a Lagrangian grid. When we consider momentum conservation, if the density terms in Equation (3.2) are absorbed into integration variable by defining $\mathrm{d} m=\rho \mathrm{d} y$, then the momentum conservation still holds without further complication. Here $m$ is usually referred to as column depth and its physical meaning is mass per unit area. 


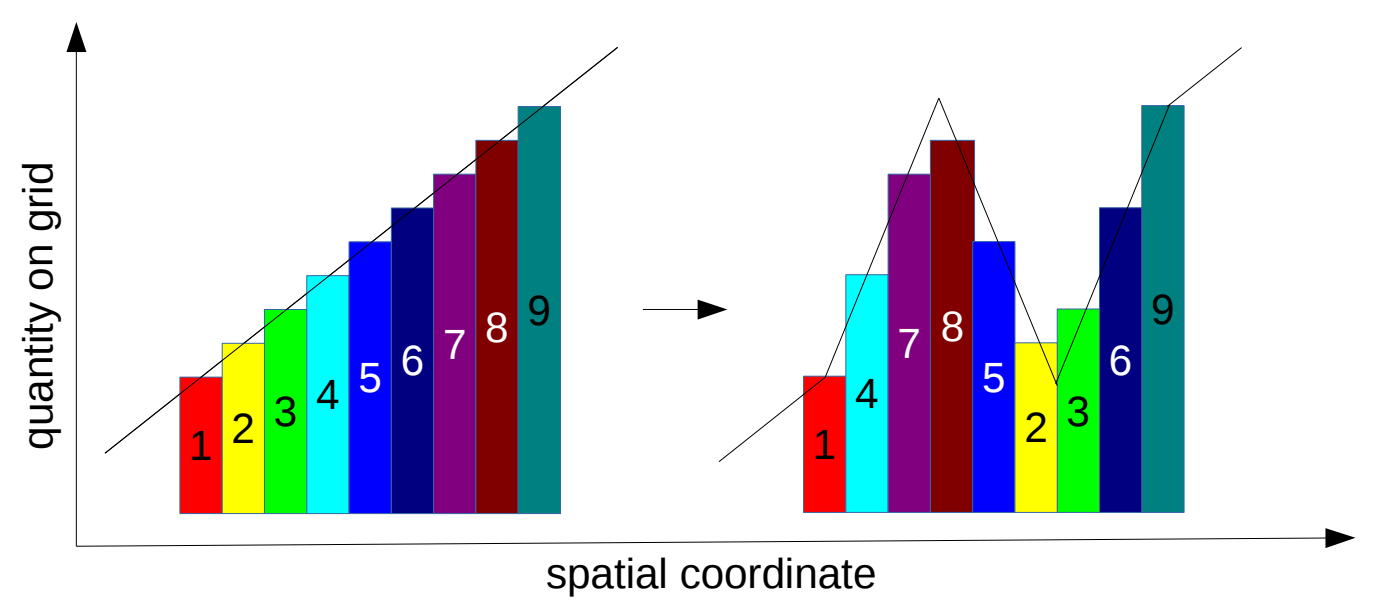

Figure 3.1: Implementation of a triplet map on a simulation grid by permutation. The solid lines are the original linear profile and the profile after a triplet map. The color bars represent the corresponding discrete values on the grid. All the bars are marked with numbers to show the permutation pattern.

Third, it is easier to implement triplet maps on a uniform Lagrangian grid. Generally speaking, there are two ways to implement a triplet map: permutation and adaptivemesh. Permutation is a simpler method that can be demonstrated by Figure 3.1. Similar to Figure 2.1, an originally linear profile of any scalar field is chosen as the example. Its value is represented by the vertical axis and the simulation grid is represented by the horizontal axis. During the mapping process, nine zones are permutated in the following fashion. Both the first and last zones stay at the original position owing to the continuity requirement. To increase the gradient by a factor of three, the rest two zones in the first segment are chosen by jumping two zones forward in each step. The second segment starts with the second to last zone (eighth zone) and proceeds by jumping two zones backward in each step. The rest three zones form the third segment similar to the first segment. Permutation is often used on a uniform grid because of its simplicity. When the grid becomes non-uniform, the adaptive-mesh method is usually adopted instead. It involves the splitting of individual zones and merging of adjacent zones in a conservative way. For our current stage of development, a uniform Lagrangian grid is chosen so that permutation is used as the operation method of a triplet map. 
Considering the environment of a neutron star surface layer in which density variation can span multiple orders of magnitude, permutations of zones cause great changes in density. In other words, the sizes of the zones change a lot after a permutation on an Eulerian grid. Thus it is almost impossible to implement triplet map without involving adaptive-mesh method. But for a Lagrangian grid with equal mass or column depth, the sizes of the zones are not affected by the density variations at all.

Despite the convenience of choosing the Lagrangian formulation, the coordinate transformation from spatial to mass/column depth is usually lengthy and can cause difficulties particularly in eddy sampling process. As introduced in Section 2.2, thinning algorithm provides an approach of accepting or rejecting an eddy trial by comparing $\lambda / \lambda^{\prime}$ with a uniformly distributed random number. Practically speaking, $\lambda$ should be a joint probability that involves not only the turbulent energy terms but also a probability function that relates to a location and length of the eddy trial. For the location, it is usually considered as a uniformly distributed function on the grid. But for the eddy length, ODT calculates it according to an assumed probability density function that was originally designated for a spatial coordinate. Although the function itself is dimensionless and pure mathematical, the result calculated carries an inherited spatial unit. If the result is used directly with a unit of mass/column depth, the density information will be missing. For example, in the region with large density profile, a reasonable length of an eddy trial on a Lagrangian grid can easily become so small on its corresponding Eulerian grid that the transformed length yields impossible or trivial case for a triplet map, i.e., smaller or equivalent to three grid spacings. For this consideration, the eddy length sampling on a Lagrangian grid is implemented in the following steps.

1. Locate the smallest spatial grid spacing by $\Delta y=\Delta m / \rho_{\max }$.

2. Calculate the total spacing of the simulation domain and split it into a uniform Eulerian grid with grid spacing as $\Delta y$.

3. Calculate the length of an eddy trial on the new grid using the probability density function.

4. Transfer the eddy region back to the Lagrangian coordinate and include all the 
affected zones as the new eddy region. Reject the eddy trial if the new region is too small to implement a non-trivial map.

The rejection of the trivial or impossible eddy trial in the last step can be considered as information lost due to different resolutions. Even when two grids share the same resolution, this problem still occur if a small eddy trial takes place at a low density region. To fully resolve this issue, the adaptive-mesh method is required.

\subsection{Flowchart}

Based on the previous introduction and discussion, a flowchart of ODT on a Lagrangian grid is concluded in Figure 3.2. Starting from initialization, ODT processes through two time systems: the eddy sampling time and the physical time. As the names indicate, the former advances according to a nonstationary Poisson process with thinning algorithm (Section 2.2) while the latter advances according to the requirements of various actual physical processes. At the beginning of the realization, a time step of eddy sampling time is calculated by

$$
\Delta t=-\frac{1}{\lambda^{\prime}} \ln U(0,1),
$$

where $\lambda^{\prime}$ is the estimated initial frequency. The location and length of an eddy trial are obtained by associating the corresponding probabilities to random numbers, respectively. After the coordinate transformation, the trial will be considered as an eddy sampling event only if it is non-trivial. Apply a triplet map in the eddy trial region and the turbulent energy terms can be calculated. Combining the aforementioned two probabilities, the overall eddy rate $\lambda$ is obtained and one reaches the select and reject process of the thinning algorithm in which the eddy is accepted with a probability $\lambda / \lambda^{\prime}$. If the trial is rejected, the eddy sampling time will advance by a new time step according to Equation (3.3) and the sampling process will repeat without any update on the physical time. Here $\lambda^{\prime}$ will be increased if $\lambda / \lambda^{\prime}$ reaches a threshold value in order to guarantee the condition of thinning algorithm, i.e., $\lambda^{\prime}>\lambda$. In other words, the average sampling time, $\tau=1 / \lambda^{\prime}$, will be reduced. If the trial is accepted, then the triplet map will be implemented in the trial region, and all the physical processes will evolve after the mapping with respect to the physical time. Once the physical time reaches the eddy 


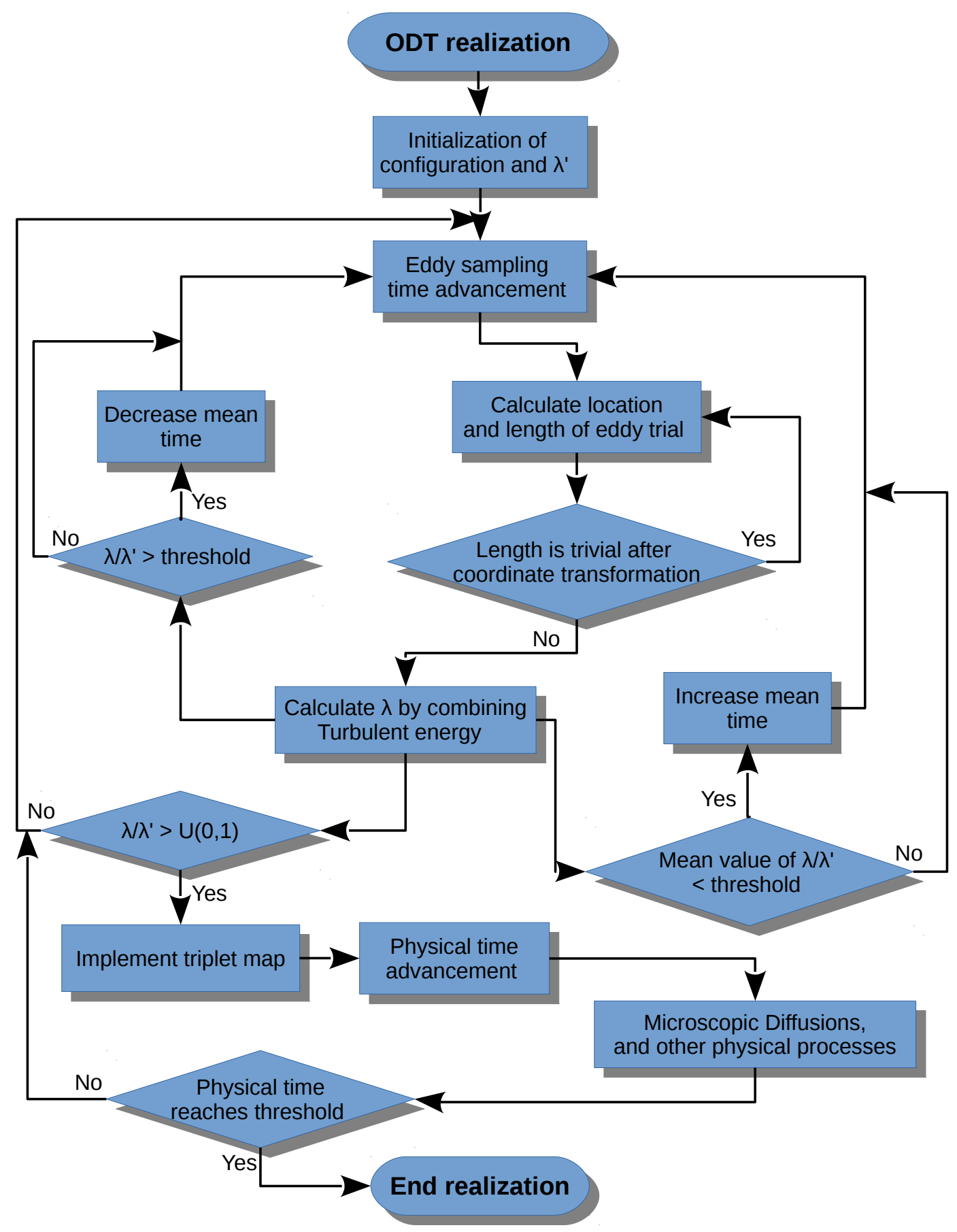

Figure 3.2: Flowchart of ODT on a Lagrangian grid. 
sampling time, ODT switches back to eddy sampling time advancement and another eddy sampling cycle starts. For each cycle, ODT updates a cycle counter and records the non-zero value of $\lambda / \lambda^{\prime}$. Once the counter reaches a certain threshold, ODT sums up all the non-zero values and calculates the average of this ratio. If the average is too small, the sampling frequency $\lambda^{\prime}$ will be decreased to reduce the sampling loads. In this way, ODT manages to couple these two time systems in one realization. To collect statistical average, ODT runs multiple realizations in parallel. It is worth noting that multiple random numbers have been applied in the process of each realization. A pseudorandom number generator named RANMAR (James 1990) is chosen because of

its efficiency and capability of generating independently disjoint sequences (Marsaglia et al. 1990). Several simple tests of its quality are shown in Appendix A.

\subsection{Rayleigh-Taylor instability}

The Rayleigh-Taylor instability (RTI) is an instability that occurs at the interface of two fluids of different densities. A typical RTI configuration is a light fluid supporting a heavy one in the gravity field or a light fluid accelerating towards a heavy one. Theory predicts that the evolution of RTI for two incompressible immiscible fluids includes two phases. In the first phase, linear stability theories predict that small amplitude perturbations at the interface grow exponentially (Chandrasekhar 1955). When the amplitude becomes comparable to its wavelength, the perturbations become asymmetric with the asymmetry increasing with the Atwood number $A=\left(\rho_{2}-\rho_{1}\right) /\left(\rho_{2}+\rho_{1}\right)$ (Dimonte et al. 2004), where $\rho_{1}$ and $\rho_{2}$ are the densities of light and heavy fluids, respectively. In the second phase, the flow grows in a self-similar way without any memory of its initial conditions. Ristorcelli \& Clark (2004) and Cook et al. (2004) found out that the growth rate can be described by the following equation

$$
\frac{\mathrm{d} h}{\mathrm{~d} t}=2(\alpha A g h)^{1 / 2} .
$$

Here $h$ is the mixing thickness and it means the height of the mixing region. Conventionally, the light fluid that penetrates the heavy one is called bubbles while the heavy fluid that penetrates the light one is called spikes. $\alpha$ is a dimensionless parameter, $g$ is 
the acceleration, and $t$ is the time. The solution to Equation (3.4) at late times is

$$
h(t)=\alpha A g t^{2} .
$$

Multiple experiments with different techniques have been carried out to measure $\alpha$. Read (1984) and Youngs (1989) used solid fuel rocket motors to accelerate a light fluid downward to a heavy fluid with its initial perturbations being random rather than a single wavelength. Andrews \& Spalding (1990) inverted a narrow tank with light fluid on top to create an unstable stratified system. By manually removing a plate as separation, Dalziel (1993) mixed two layers of fluid with heavy one on top. Pacitto et al. (2000) approached the initial perturbation by stabilizing and destabilizing the interface of two magnetic fluids with a magnetic field. Ramaprabhu \& Andrews (2004) used water of different temperatures flowing through a channel with splitter for the set-up. From these experiments, $\alpha$ is found in the range $0.03<\alpha<0.07$ Cook \& Youngs 2009). Compared with experiments, simulations of RTI have an obvious advantage on the initial perturbation set-up, which is usually categorized into single-mode and multimode. In detail, simulations usually use perturbations with wavelengths much smaller than $h$ so that the exponential growth in Equation 3.4 won't contaminate the self-similar growth. Besides, simulations can expand their domains such that the boundary influence is negligible. But the calculation turns costly since the large lengthscale change it has to cover. Dimonte et al. (2004) summarized the range of $\alpha$ from simulation results: $0.02<\alpha<0.08$.

In this section I present RTI simulation using ODT for the purpose of testing ODT. The initial set-up is similar to that used in Dimonte et al. (2004) but transferred to a grid of mass with reversed acceleration. As shown in Figure 3.3, a layer of idea gas of density $\rho_{2}=3 \mathrm{~g} / \mathrm{cm}^{3}$ (shaded area) accelerates towards a layer of gas of density $\rho_{1}=1 \mathrm{~g} / \mathrm{cm}^{3}$ with an acceleration $\mathrm{g}=2 \mathrm{~cm} / \mathrm{s}^{2}$. The simulation domain has increased by a factor of three with 1500 grid points included to avoid possible boundary influence. 3D simulations usually perturb the interface by different modes, which involves the difficulty on band-limiting of initial condition. Several simulations indicate that there is a sensitive relation between the initial condition and results Cook \& Dimotakis 2001; Glimm et al. 2001; Dimonte et al. 2004). ODT uses a sharp interface instead and the initial development of eddies is obtained by eddy sampling. Considering a trial eddy 


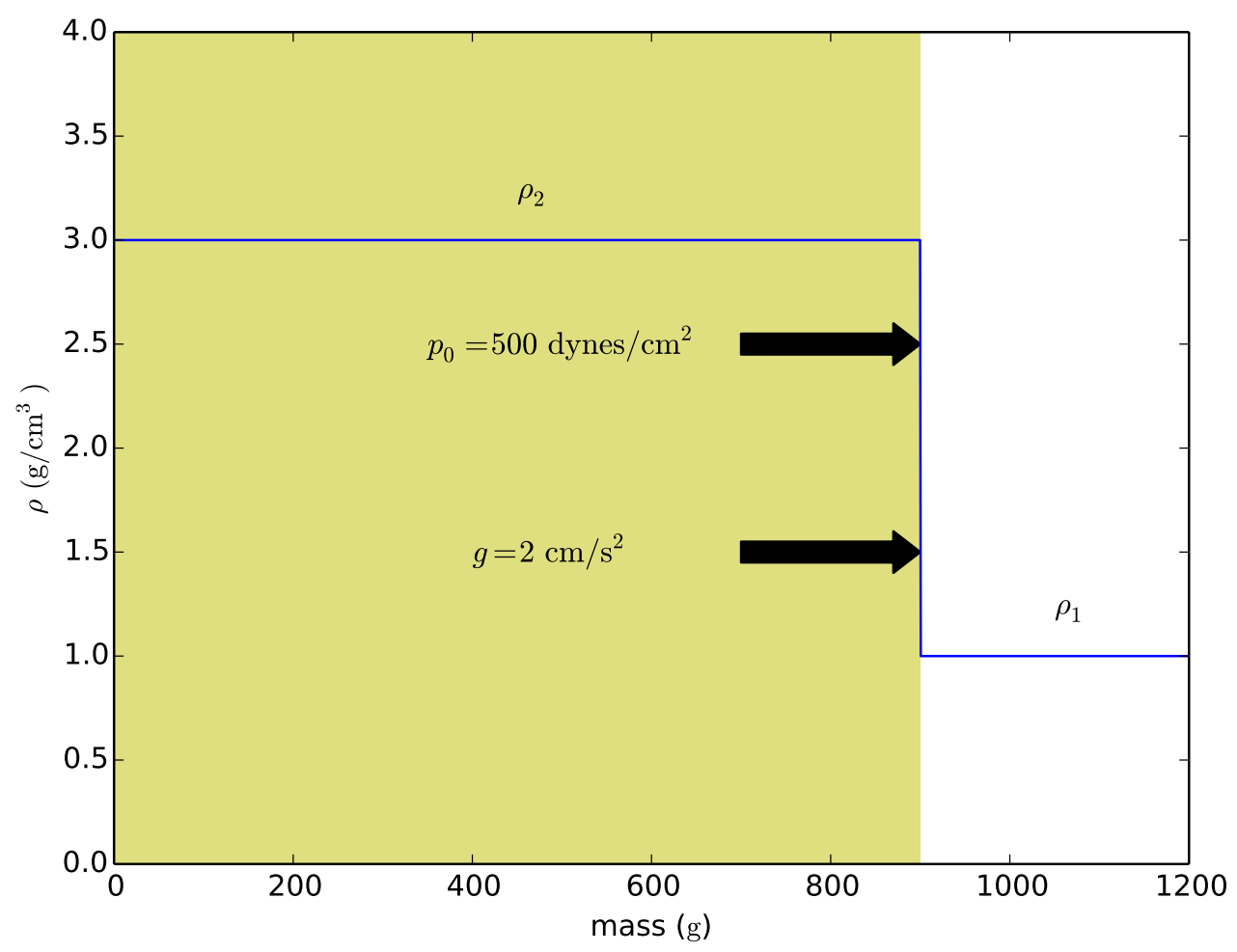

Figure 3.3: Initial density profile of RTI simulation. A layer of idea gas of density $\rho_{1}=1 \mathrm{~g} / \mathrm{cm}^{2}$ supports a layer of idea gas of density $\rho_{2}=3 \mathrm{~g} / \mathrm{cm}^{3}$ against an acceleration field $g=2 \mathrm{~cm} / \mathrm{s}^{2}$. A sharp interface is implemented without any initial perturbation. The simulation regions of densities are set with unequal sizes in order to match the corresponding set-up in Eulerian coordinates that are used in 3D DNS studies.

across the interface, the energy conservation implies that

$$
\begin{aligned}
\left(\frac{1}{2} \int \mathbf{u}_{\mathbf{f}}^{2} \mathrm{~d} m+\int g y_{f} \mathrm{~d} m+\int \frac{5 R T_{f}}{2 \mu_{f}} \mathrm{~d} m\right) & -\left(\frac{1}{2} \int \mathbf{u}^{2} \mathrm{~d} m+\int g y \mathrm{~d} m+\int \frac{5 R T}{2 \mu} \mathrm{d} m\right) \\
& =\int p\left(\frac{1}{\rho_{f}}-\frac{1}{\rho}\right) \mathrm{d} m .
\end{aligned}
$$

The terms in each bracket on the left hand side of the equation are the kinetic energy, potential energy, and thermal energy, respectively. $\mathbf{u}$ is the velocity vector, $m$ is the mass or column depth, $y$ is the length, $R$ is the gas constant, $T$ is the temperature, $\mu$ is 
the molar mass of the chosen ideal gas (hydrogen and helium), and $p$ is the pressure. The mapped quantities are represented by a subscript for convenience and $\mathbf{u}_{\mathbf{f}}=\mathbf{u}+\mathbf{c} K(m)$. The integration interval is the trial eddy range $\left[m_{0}, m_{0}+l_{m}\right]$. Similar to Equation (2.5), the solution is

$$
c_{i}=\frac{27}{4 l_{m}}\left(-u_{i, K}+\operatorname{sgn}\left(u_{i, K}\right) \sqrt{\frac{1}{3}\left(\sum_{i=1}^{3} u_{i, K}^{2}-\frac{8}{27 l_{m}} \Delta E\right)}\right),
$$

where

$$
\Delta E=g \int\left(y_{f}-y\right) \mathrm{d} m+\frac{5 R}{2} \int\left(\frac{T_{f}}{\mu_{f}}-\frac{T}{\mu}\right) \mathrm{d} m-\int p\left(\frac{1}{\rho_{f}}-\frac{1}{\rho}\right) \mathrm{d} m,
$$

and

$$
u_{i, K} \equiv \frac{1}{l_{m}^{2}} \int u_{f} K(m) \mathrm{d} m
$$

A factor of $1 / 3$ inside the square root indicates isotropic redistribution of turbulent energy. The eddy rate distribution therefore is

$$
\lambda=\frac{C}{l^{3}} \sqrt{\sum_{i=1}^{3} u_{i, K}^{2}-\frac{8}{27 l_{m}} \Delta E-Z \frac{\nu^{2}}{l^{2}}} .
$$

It is worth noting that $l$ is the corresponding spatial length of $l_{m}$. The formulation of Equation (3.10) is generalized to compressible fluid with an adiabatic triplet map assumption. Another approach with Boussinesq approximation is similar to Equation 2.11) and is used in Gonzalez-Juez et al. (2013)

With properly chosen $C$ and $Z$ parameters, any eddy trial across the interface is likely to be accepted owing to the highly unstable configuration. Sometimes an unphysically large eddy can occur. ODT introduces a large-eddy-suppression mechanism to avoid these eddies of sizes exceeding the mixing thickness. In general there are three methods in publications to date. The first method introduces one more free parameter $\beta$ to explicitly constrain the eddy turnover time scale $\tau=C /\left(l^{2} \lambda\right)$, namely $\beta \tau<t$, where $t$ is the given elapsed time (Kerstein \& Dreeben 2000). The second method adds another way to evaluate the eddy rate distribution to Equation 3.10 by replacing each velocity profile with a linear profile (Kerstein et al. 2001). Each linear profile has a slope that is equal to the median value of the corresponding velocity gradient magnitude within the eddy region. The final eddy rate is chosen as the smaller value of these 
two evaluated eddy rates. This method eliminates the case when more than half of the eddy region has a flat velocity profile. The third method doesn't introduce any new parameter or new evaluation method. In detail, it equally divides the eddy interval into three subintervals: $\left[m_{0}, m_{0}+l / 3\right],\left[m_{0}+l / 3, m_{0}+2 l / 3\right]$, and $\left[m_{0}+2 l / 3, m_{0}+l\right]($ Ashurst \& Kerstein 2005). Then it evaluates an eddy rate within each subinterval according to Equation (3.10) but replaces the viscous penalty term with the one calculated from the overall interval $\left[m_{0}, m_{0}+l\right]$. If any of these three rates is zero, the eddy trial is suppressed. The overall eddy rate otherwise is calculated and accepted. This method rejects eddy trials with their unstable regions concentrated within one or two subintervals. Thus their eddy lengths should be reduced to sizes that encompass the actually turbulent region. Compared with the first two methods, the last one is most robust and with least arbitrary, so it is adopted in this dissertation.

Three diffusion processes are included and they can be written as follows:

$$
\begin{aligned}
\frac{\partial T}{\partial t} & =\rho \frac{\partial}{\partial m}\left(\rho \kappa_{T} \frac{\partial T}{\partial m}\right), \\
\frac{\partial Y_{i}}{\partial t} & =\rho \frac{\partial}{\partial m}\left(\rho \kappa_{Y} \frac{\partial Y_{i}}{\partial m}\right), \\
\frac{\partial u_{i}}{\partial t} & =\rho \frac{\partial}{\partial m}\left(\rho \nu \frac{\partial u_{i}}{\partial m}\right),
\end{aligned}
$$

where $Y_{i}$ is the molar fraction of isotope $i, \kappa_{T}$ is the thermal diffusivity, $\kappa_{Y}$ is the molar diffusion coefficient, and $\nu$ is the kinematic viscosity. The numerical solution to these equations will be discussed in Section 3.5. Combining with the ideal gas equation of state

$$
P=\frac{\rho R T}{\mu},
$$

all the properties can be solved as a function of time.

The density profile of a chosen single realization is shown in Figure 3.4 . Each vertical discontinuous line in color represents an instantaneous mapping event. The turbulent region initiates from the interface shortly after the simulation starts and develops into two fluids as the sizes of bubbles and spikes grow. Because each realization is independent, multiple realizations calculation can be easily parallelized using MPI. Figure 3.5 shows the averaged mixing thickness of 1024-realization simulations (represented by dots). The 


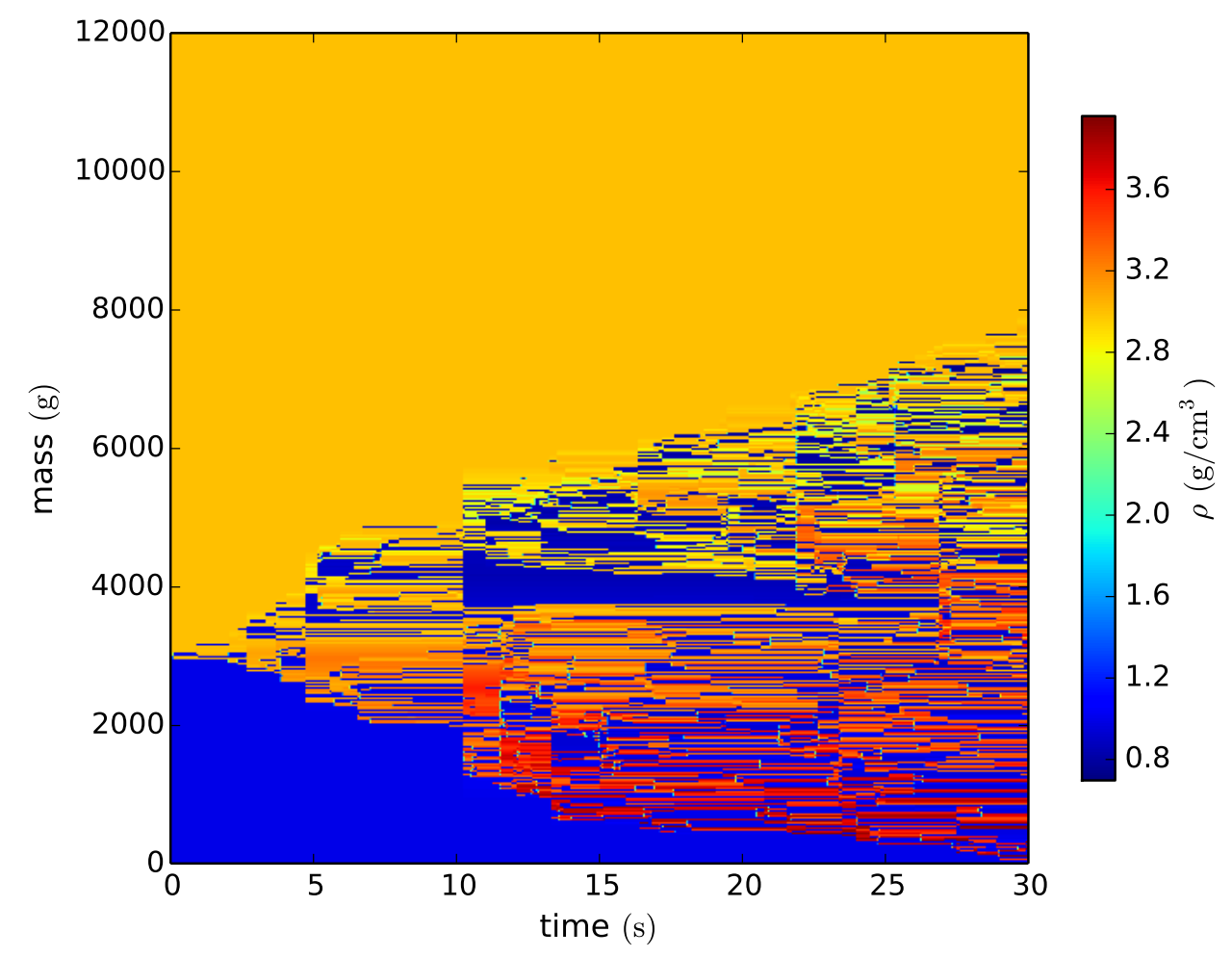

Figure 3.4: Density evolution of RTI. Evolution of a single realization profile demonstrates the instantaneous mapping events as discontinuous lines in color. The turbulence initiates quickly around the interface after the simulation starts. Bubbles and spikes develop as the turbulent cascade structure forms.

results are compared with those of FLASH (Calder et al. 2002) which are represented by triangles. The bubbles are in blue color while the spikes are in red color. A vertical dashed line at $t=5 \mathrm{~s}$ marks the early developing phase with a rapidly changing slope. Similar to Calder et al. (2002), $h$ is measured by comparing the density deviation from the initial profile by more than $1 \%$. Overall, ODT results show a nice linear relationship between $h$ and $A g t^{2}$. A particular set of parameters, i.e., $C=5 \times 10^{-4}$ and $Z=0.5$, is chosen to fit the results of FLASH with an emphasis particularly on spike height. In detail, ODT results are $\alpha_{b}=0.016$ for the bubbles and $\alpha_{s}=0.028$ for the spikes, while FLASH results are $\alpha_{b}=0.024$ and $\alpha_{s}=0.030$. If the first five seconds are neglected, 


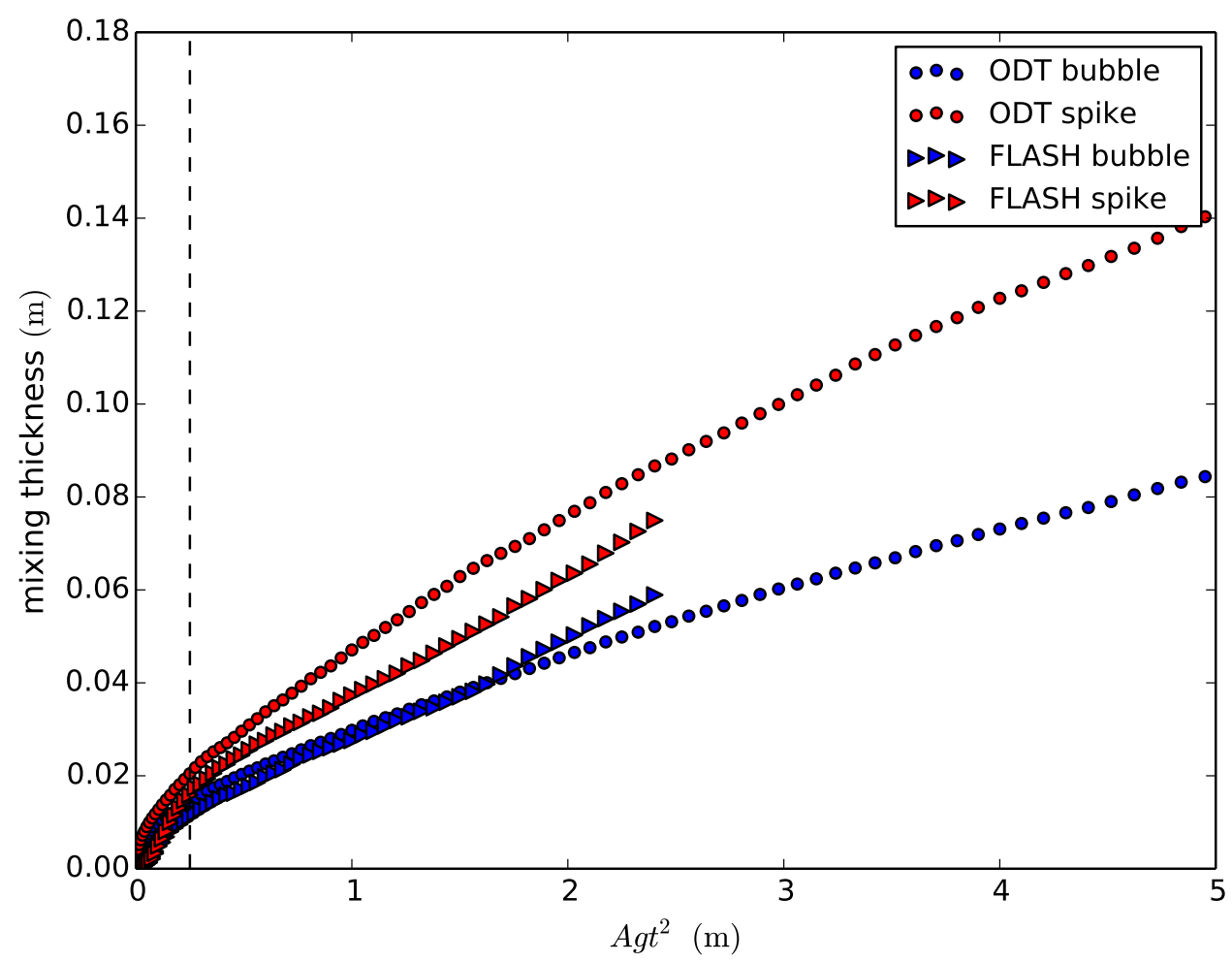

Figure 3.5: Mixing thickness of RTI. The results are obtained by averaging over the density profiles of 1024 realizations. Bubbles and spikes heights are computed by measuring the density deviation from the initial profile by more than $1 \%$. A set of parameter $(C, Z)=\left(5 \times 10^{-4}, 0.5\right)$ is chosen to fit the spikes heights of FLASH, i.e., $\alpha_{s}=0.026$ if the first $5 \mathrm{~s}$ (vertical dashed line) that involves rapid slope changing is neglected.

ODT yields $\alpha_{b}=0.014$ and $\alpha_{s}=0.026$, while Flash yields $\alpha_{b}=0.021$ and $\alpha_{s}=0.026$.

There is no special reason for this choice of $C$ and $Z$ since another set of parameter can fit the bubble height equally well. A noticeable difference is that $\alpha$ for the bubbles in ODT is smaller than that in FLASH. As mentioned in Section 3.1, the sampling statistics are implemented by transforming everything back to an Eulerian coordinate. So the masses of two idea gases have a ratio 3:1 in the initial configuration to match the equal volume set-up of 3D simulation (Calder et al. 2002). In the Eulerian coordinate, this sampling mechanism indicates an almost equal development of bubbles and spikes. But in the Lagrangian coordinate, eddy lengths of bubbles are bigger than those of spikes. 
Large-eddy-suppression mechanism tends to accept eddy trials with smaller eddy sizes, which indicates that more eddy trials accepted for spikes than bubbles. As a result, the development of spikes is faster than that of bubbles.

The purpose of this application is to test the configuration in the Lagrangian coordinate, so the choice of $C$ and $Z$ is decided to match a DNS result. Gonzalez-Juez et al. (2013) did a survey in the parameter space for non-reactive RTI using Boussinesq approximation and found that $0.02 \leq \alpha \leq 0.08$ for different sets of $C$ and $Z$. And a particular set of parameters can fit the DNS result $\alpha=0.03$ very well. Because parameters are not configuration independent, e.g., a change in the size of simulation domain will result in changes in the parameters, it is not necessary to compare parameters for different configurations. In conclusion, the test shows that ODT on a Lagrangian grid is capable of simulating RTI in good agreement with DNS results.

\subsection{Equation of state}

The equation of state, or EOS, of an ideal gas is adequate for the RTI problem. But when it comes to neutron stars, a stellar EOS must be included to the calculations. In ODT, the Helmholtz EOS (Timmes \& Swesty 2000) is adopted because of its accuracy and speed for our applications in the rest of this dissertation. The Helmholtz EOS chooses Helmholtz free energy $F(\rho, T)$ for thermodynamic consistency. $F(\rho, T)$ and its

eight partial derivatives are tabulated with the density $\rho$ ranging from $10^{-12} \mathrm{~g} / \mathrm{cm}^{3}$ to $10^{15} \mathrm{~g} / \mathrm{cm}^{3}$, and with the temperature $T$ ranging from $10^{3} \mathrm{~K}$ to $10^{13} \mathrm{~K}$ in the package on Frank Timmes' webpage (Timmes 2015b). A biquintic Hermite polynomial is used as the interpolating function to gain the continuity of the pressure, entropy, and internal energy derivatives, although the interpolation scheme is independent of the chosen function. An electron-position EOS table is accurately constructed based on the Helmholtz free energy and its derivatives, and the part of ions and photons are calculated analytically because of their simplicity. Practically speaking, the EOS yields most of the other thermodynamic quantities once $(\rho, T)$ are given.

In the application of ODT, the pair $(\rho, T)$, however, is not always given. In fact, neither of them is known in the assumed adiabatic mapping process and the isobaric process that involves nuclear reactions. Besides, our choice of uniform mass grid and 
hydrostatic equilibrium assumption guarantees that the pressure is always explicit

$$
P=\sum_{i} m_{i} g
$$

where $i$ is the grid number starting from the top. So most of our applications combine one of $(\rho, T)$ with $P$. Considering the adiabatic process, the specific entropy and the pressure $(s, P)$ are known before and after a mapping. The thermodynamic potential corresponding to $(s, P)$ is the specific enthalpy $h$, defined as

$$
\mathrm{d} h=T \mathrm{~d} s+\frac{1}{\rho} \mathrm{d} P .
$$

It would be ideal if a similar table of $h(s, p)$ could be constructed and the other thermodynamic quantities can be quickly obtained with thermodynamic consistency by a given $(s, P)$ pair. It is, however, not so easy to achieve because of the following two reasons. First, there is no guarantee that a specific $(s, P)$ always corresponds to a valid thermodynamic solution, which indicates that some mechanism must be included first to determine the validity of the specific pair. In general, it is difficult to accomplish. Second, the Helmholtz EOS on the $(\rho, T)$ basis is a rectangular table, which makes it convenient to locate a point with a fast one-line hash table look-up. On the contrary, a table on the $(s, P)$ basis is unlikely to be rectangular, which indicates a more expensive search mechanism is required. Based on these considerations, we use a more straightforward approach: the Newton-Raphson method.

The Newton-Raphson method is a powerful root-finding algorithm with a quadratic converging rate (Press et al. 1993). The basic idea of 1D Newton-Raphson method can be summarized as follows. Consider an equation of variable $x$,

$$
f(x)=0,
$$

with its solution $x=x^{*}$. Starting with a guess $x=x_{0}$, the Taylor expansion shows that

$$
f\left(x_{0}+\delta x\right)=f\left(x_{0}\right)+f^{\prime}\left(x_{0}\right) \delta x+\ldots
$$

Keeping the Taylor series only to first order and setting $f\left(x_{0}+\delta x\right)=0$, an expression for $\delta x \equiv \delta x_{0}$ can be found

$$
\delta x_{0}=\frac{f\left(x_{0}+\delta x\right)-f\left(x_{0}\right)}{f^{\prime}\left(x_{0}\right)}=-\frac{f\left(x_{0}\right)}{f^{\prime}\left(x_{0}\right)} .
$$


So a new $x=x_{1}$ can be adjusted by

$$
x_{1}=x_{0}+\delta x_{0}=x_{0}-\frac{f\left(x_{0}\right)}{f^{\prime}\left(x_{0}\right)},
$$

which should be closer to the root $x^{*}$. Repeat this process $\mathrm{k}$ times with

$$
\delta x_{k}=-\frac{f\left(x_{k}\right)}{f^{\prime}\left(x_{k}\right)},
$$

and

$$
x_{k+1}=x_{k}-\frac{f\left(x_{k}\right)}{f^{\prime}\left(x_{k}\right)},
$$

until $\left|x_{k+1}-x^{*}\right|$ is small enough to fulfill a certain requirement. Then $x_{k+1}$ is considered as the numerical root of Equation 3.17).

In our ODT application, 1D Newton-Raphson method includes two cases: $(P, T)$ and $(P, \rho)$. Because $P$ is always known according to Equation (3.15), replace $x$ with $T$ and $\rho$, respectively, to solve

$$
f(\rho)=P\left(\rho, T^{*}\right)-P^{*}=0,
$$

and

$$
f(T)=P\left(T, \rho^{*}\right)-P^{*}=0 .
$$

Here the given quantities are marked with '*'. The Helmholtz EOS provides properties $\frac{\partial P}{\partial \rho}$ and $\frac{\partial P}{\partial T}$, so the iterations can be processed as

$$
\rho_{k+1}=\rho_{k}-\frac{\Delta P\left(\rho_{k}\right)}{\frac{\partial P\left(\rho_{k}\right)}{\partial \rho}},
$$

and

$$
T_{k+1}=T_{k}-\frac{\Delta P\left(T_{k}\right)}{\frac{\partial P\left(T_{k}\right)}{\partial T}},
$$

where

$$
\begin{aligned}
& \Delta P\left(\rho_{k}\right) \equiv P\left(\rho_{k}, T^{*}\right)-P^{*}, \\
& \Delta P\left(T_{k}\right) \equiv P\left(T_{k}, \rho^{*}\right)-P^{*},
\end{aligned}
$$

and $k=0,1,2, \ldots$ 
1D Newton-Raphson algorithm can be easily generalized to 2D case. In ODT, there are also two cases that involve a $2 \mathrm{D}$ solver: $(s, P)$ and $(h, P)$. Here I derive for the former first. Consider the following equations

$$
\begin{aligned}
& f\left(\rho_{k}, T_{k}\right)=s\left(\rho_{k}, T_{k}\right)-s^{*}=0, \\
& g\left(\rho_{k}, T_{k}\right)=P\left(\rho_{k}, T_{k}\right)-P^{*}=0 .
\end{aligned}
$$

Expand them around $\left(\rho_{k}, T_{k}\right)$ with $\left(\delta \rho_{k}, \delta T_{k}\right)$ to first order, then

$$
\begin{aligned}
& f\left(\rho_{k}+\delta \rho_{k}, T_{k}+\delta T_{k}\right)=f\left(\rho_{k}, T_{k}\right)+\frac{\partial f\left(\rho_{k}, T_{k}\right)}{\partial \rho} \delta \rho_{k}+\frac{\partial f\left(\rho_{k}, T_{k}\right)}{\partial T} \delta T_{k}, \\
& g\left(\rho_{k}+\delta \rho_{k}, T_{k}+\delta T_{k}\right)=g\left(\rho_{k}, T_{k}\right)+\frac{\partial g\left(\rho_{k}, T_{k}\right)}{\partial \rho} \delta \rho_{k}+\frac{\partial g\left(\rho_{k}, T_{k}\right)}{\partial T} \delta T_{k} .
\end{aligned}
$$

By setting

$$
\begin{aligned}
& f\left(\rho_{k}+\delta \rho_{k}, T_{k}+\delta T_{k}\right)=0, \\
& g\left(\rho_{k}+\delta \rho_{k}, T_{k}+\delta T_{k}\right)=0,
\end{aligned}
$$

and combining

$$
\frac{\partial f}{\partial \rho}=\frac{\partial s}{\partial \rho}, \frac{\partial f}{\partial T}=\frac{\partial s}{\partial T}, \frac{\partial g}{\partial \rho}=\frac{\partial P}{\partial \rho}, \frac{\partial g}{\partial \rho}=\frac{\partial P}{\partial \rho},
$$

a matrix of $\left(\delta \rho_{k}, \delta T_{k}\right)$ is obtained as

$$
\left(\begin{array}{cc}
\frac{\partial s\left(\rho_{k}, T_{k}\right)}{\partial \rho} & \frac{\partial s\left(\rho_{k}, T_{k}\right)}{\partial T} \\
\frac{\partial P\left(\rho_{k}, T_{k}\right)}{\partial \rho} & \frac{\partial P\left(\rho_{k}, T_{k}\right)}{\partial T}
\end{array}\right)\left(\begin{array}{l}
\delta \rho_{k} \\
\delta T_{k}
\end{array}\right)=\left(\begin{array}{c}
\Delta s\left(\rho_{k}, T_{k}\right) \\
\Delta P\left(\rho_{k}, T_{k}\right)
\end{array}\right),
$$

where $\Delta s\left(\rho_{k}, T_{k}\right) \equiv s^{*}-s\left(\rho_{k}, T_{k}\right), \Delta P\left(\rho_{k}, T_{k}\right) \equiv P^{*}-P\left(\rho_{k}, T_{k}\right)$. The solution is

$$
\begin{aligned}
& \delta \rho_{k}= \frac{\frac{\partial P\left(\rho_{k}, T_{k}\right)}{\partial T} \Delta s\left(\rho_{k}, T_{k}\right)-\frac{\partial s\left(\rho_{k}, T_{k}\right)}{\partial T} \Delta P\left(\rho_{k}, T_{k}\right)}{\frac{\partial s\left(\rho_{k}, T_{k}\right)}{\partial \rho} \frac{\partial P\left(\rho_{k}, T_{k}\right)}{\partial T}-\frac{\partial P\left(\rho_{k}, T_{k}\right)}{\partial \rho} \frac{\partial s\left(\rho_{k}, T_{k}\right)}{\partial T}}, \\
& \delta T_{k}= \frac{\partial s\left(\rho_{k}, T_{k}\right)}{\partial \rho} \Delta P\left(\rho_{k}, T_{k}\right)-\frac{\partial P\left(\rho_{k}, T_{k}\right)}{\partial \rho} \Delta s\left(\rho_{k}, T_{k}\right) \\
& \frac{\partial s\left(\rho_{k}, T_{k}\right)}{\partial \rho} \frac{\partial P\left(\rho_{k}, T_{k}\right)}{\partial T}-\frac{\partial P\left(\rho_{k}, T_{k}\right)}{\partial \rho} \frac{\partial s\left(\rho_{k}, T_{k}\right)}{\partial T}
\end{aligned}
$$

Similar to Equation (3.26), the iteration pattern is

$$
\begin{gathered}
\rho_{k+1}=\rho_{k}+\delta \rho_{k}, \\
T_{k+1}=T_{k}+\delta T_{k} .
\end{gathered}
$$


When we need to solve for $(h, P)$, most of the derivations are the same but with $h$ replaced by $s$. Because the Helmholtz EOS doesn't include properties related to $h$ explicitly, $\frac{\partial h}{\partial \rho}$ and $\frac{\partial h}{\partial T}$ need some extra effort. According to the definition,

$$
h=e+\frac{P}{\rho},
$$

where $e$ is the specific internal energy. Take the partial derivatives of the equation above with respect to $\rho$ and $T$ separately, then

$$
\begin{aligned}
& \frac{\partial h}{\partial \rho}=\frac{\partial e}{\partial \rho}+\frac{1}{\rho} \frac{\partial P}{\partial \rho}-\frac{P}{\rho^{2}}, \\
& \frac{\partial h}{\partial T}=\frac{\partial e}{\partial T}+\frac{1}{\rho} \frac{\partial P}{\partial T} .
\end{aligned}
$$

Now the equations for $(h, P)$ corresponding to Equation (3.34) can be written as follows:

$$
\begin{gathered}
\delta \rho_{k}=\frac{\frac{\partial P\left(\rho_{k}, T_{k}\right)}{\partial T} \Delta h\left(\rho_{k}, T_{k}\right)-\left(\frac{\partial e\left(\rho_{k}, T_{k}\right)}{\partial T}+\frac{1}{\rho_{k}} \frac{\partial P\left(\rho_{k}, T_{k}\right)}{\partial T}\right) \Delta P\left(\rho_{k}, T_{k}\right)}{\left(\frac{\partial e\left(\rho_{k}, T_{k}\right)}{\partial \rho} \frac{\partial P\left(\rho_{k}, T_{k}\right)}{\partial T}-\frac{\partial P\left(\rho_{k}, T_{k}\right)}{\partial \rho} \frac{\partial e\left(\rho_{k}, T_{k}\right)}{\partial T}\right)-\frac{P\left(\rho_{k}, T_{k}\right)}{\rho_{k}^{2}} \frac{\partial P\left(\rho_{k}, T_{k}\right)}{\partial T}}, \\
\delta T_{k}=\frac{\left(\frac{\partial e\left(\rho_{k}, T_{k}\right)}{\partial \rho}+\frac{1}{\rho_{k}} \frac{\partial P\left(\rho_{k}, T_{k}\right)}{\partial \rho}-\frac{P\left(\rho_{k}, T_{k}\right)}{\rho_{k}^{2}}\right) \Delta P\left(\rho_{k}, T_{k}\right)-\frac{\partial P\left(\rho_{k}, T_{k}\right)}{\partial \rho} \Delta h\left(\rho_{k}, T_{k}\right)}{\left(\frac{\partial e\left(\rho_{k}, T_{k}\right)}{\partial \rho} \frac{\partial P\left(\rho_{k}, T_{k}\right)}{\partial T}-\frac{\partial P\left(\rho_{k}, T_{k}\right)}{\partial \rho} \frac{\partial e\left(\rho_{k}, T_{k}\right)}{\partial T}\right)-\frac{P\left(\rho_{k}, T_{k}\right)}{\rho_{k}^{2}} \frac{\partial P\left(\rho_{k}, T_{k}\right)}{\partial T}} .
\end{gathered}
$$

Here $\Delta h\left(\rho_{k}, T_{k}\right) \equiv h^{*}-e\left(\rho_{k}, T_{k}\right)-\frac{P\left(\rho_{k}, T_{k}\right)}{\rho_{k}}$, where the definition of $h$ (Equation 3.36 has been substituted. Since the internal energy and its derivatives can be drawn directly from the EOS, and $h^{*}$ can be derived from isobaric process (see Section 4.3), the information in Equation (3.38) is completed.

It is worth noting that multidimensional Newton-Raphson method cannot guarantee convergence to a root (Press et al. 1993). For $(h, P)$, the root finding usually fails if the step $(\delta \rho, \delta T)$ is too big and the initial guess $\left(\rho_{0}, T_{0}\right)$ is not sufficiently good. Because $(h, P)$ is designated for heat generated during nuclear reactions, which is usually small owing to the constraint on time step, a search in nearby region with reduced step $\left(\delta \rho^{\prime}, \delta T^{\prime}\right)$ turns out to be efficient and accurate. In detail,

$$
\left(\delta \rho_{k}^{\prime}, \delta T_{k}^{\prime}\right)=\omega\left(\delta \rho_{k}, \delta T_{k}\right)
$$




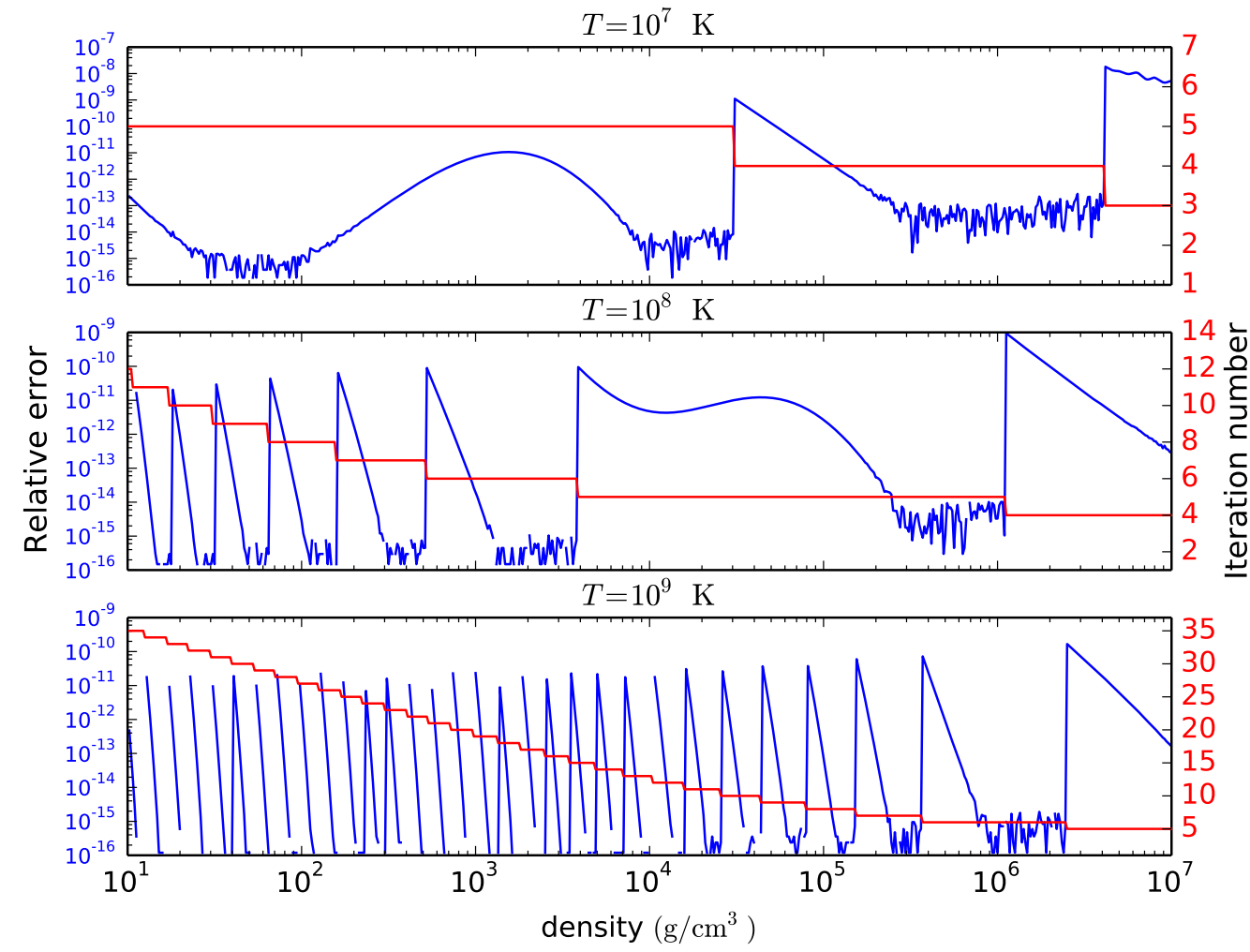

Figure 3.6: Relative errors of $T$ given $(P, \rho)$ and iterations number.

where

$$
\omega= \begin{cases}1 & \text { if } r \leq 0.2, \\ \frac{0.2}{r} & \text { if } r>0.2,\end{cases}
$$

with $r$ as the maximum value of $\left(\frac{\delta \rho_{k}}{\rho_{k}}, \frac{\delta T_{k}}{T_{k}}\right)$. Therefore, the iteration pattern is

$$
\begin{aligned}
& \rho_{k+1}=\rho_{k}+\omega \delta \rho_{k}, \\
& T_{k+1}=T_{k}+\omega \delta T_{k} .
\end{aligned}
$$

The tests for the four cases of the EOS solver are shown in Figures 3.6 to 3.9 . Each figure shows the absolute value of relative differences with blue color on the left 


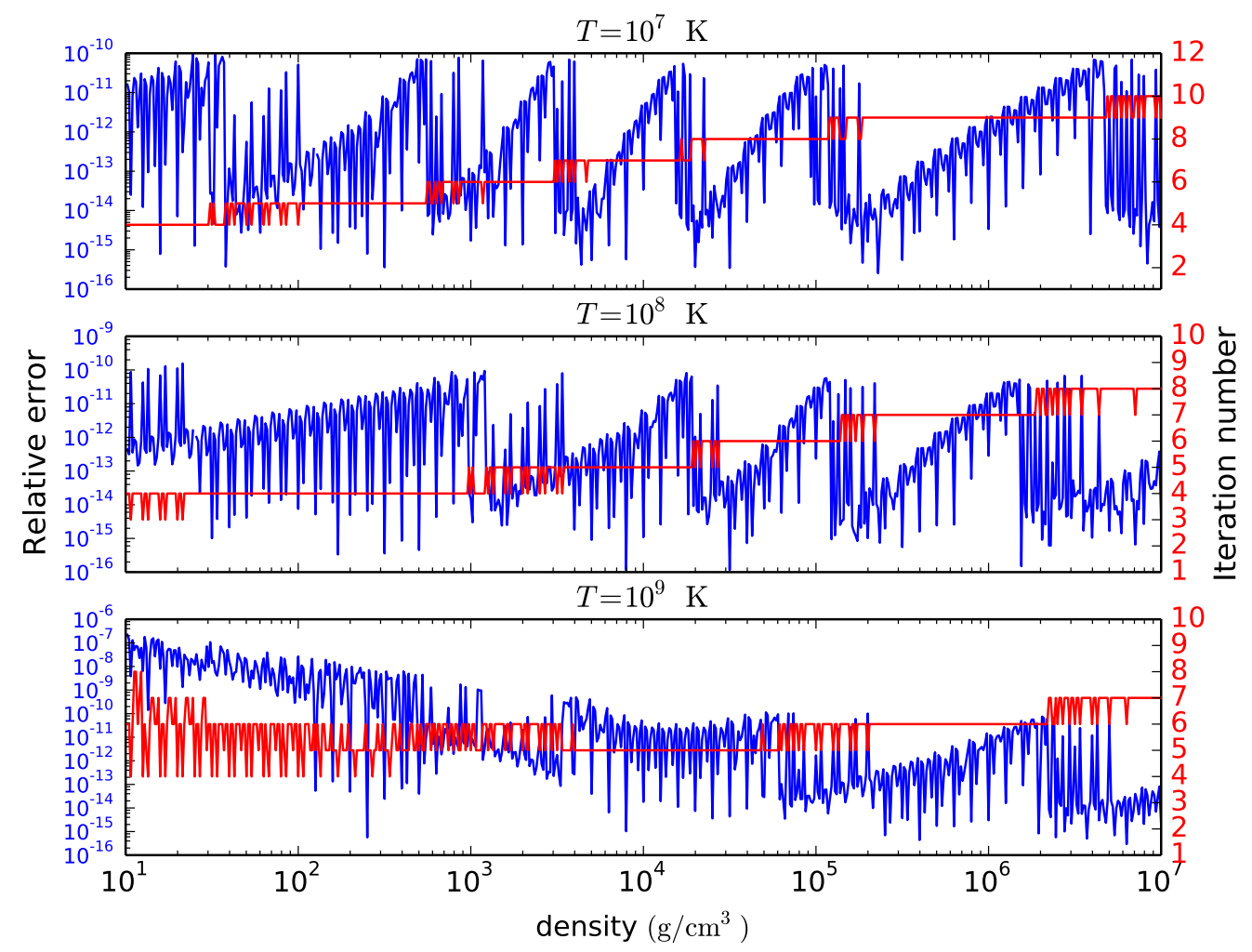

Figure 3.7: Relative errors of $\rho$ given $(P, T)$ and iterations number.

axis, and the number of iterations used to find the root during the process with red color on the right axis. Each panel in the figure corresponds to temperatures $10^{7} \mathrm{~K}$, $10^{8} \mathrm{~K}$, and $10^{8} \mathrm{~K}$, respectively. These temperatures are chosen to cover the range in which nuclear reactions are mostly active during an XRB. The densities range from $10^{1} \mathrm{~g} / \mathrm{cm}^{3}$ to $10^{7} \mathrm{~g} / \mathrm{cm}^{3}$ on a logarithmic scale. Usually a density range from $10^{4} \mathrm{~g} / \mathrm{cm}^{3}$ to $10^{7} \mathrm{~g} / \mathrm{cm}^{3}$ is sufficient for XRBs, here small density range is added to include the surface expansion during a PRE burst. The figures are plotted with respect to $(\rho, T)$ because this pair yields a nice rectangular table. If one of the pair is the root, like $T$ in Figure 3.6 or $\rho$ in Figure 3.7, the given pair, $(P, \rho)$ or $(P, T)$, is first calculated for each $(\rho, T)$ in the table. Then use the corresponding solver to obtain $T$ or $\rho$ by an initial guess of $T_{0}=10^{3} \mathrm{~K}$ or $\rho_{0}=0.1 \mathrm{~g} / \mathrm{cm}^{3}$. Finally, we compare the root with the known grid point to obtain the relative difference. The same method applies to $(P, s)$ 


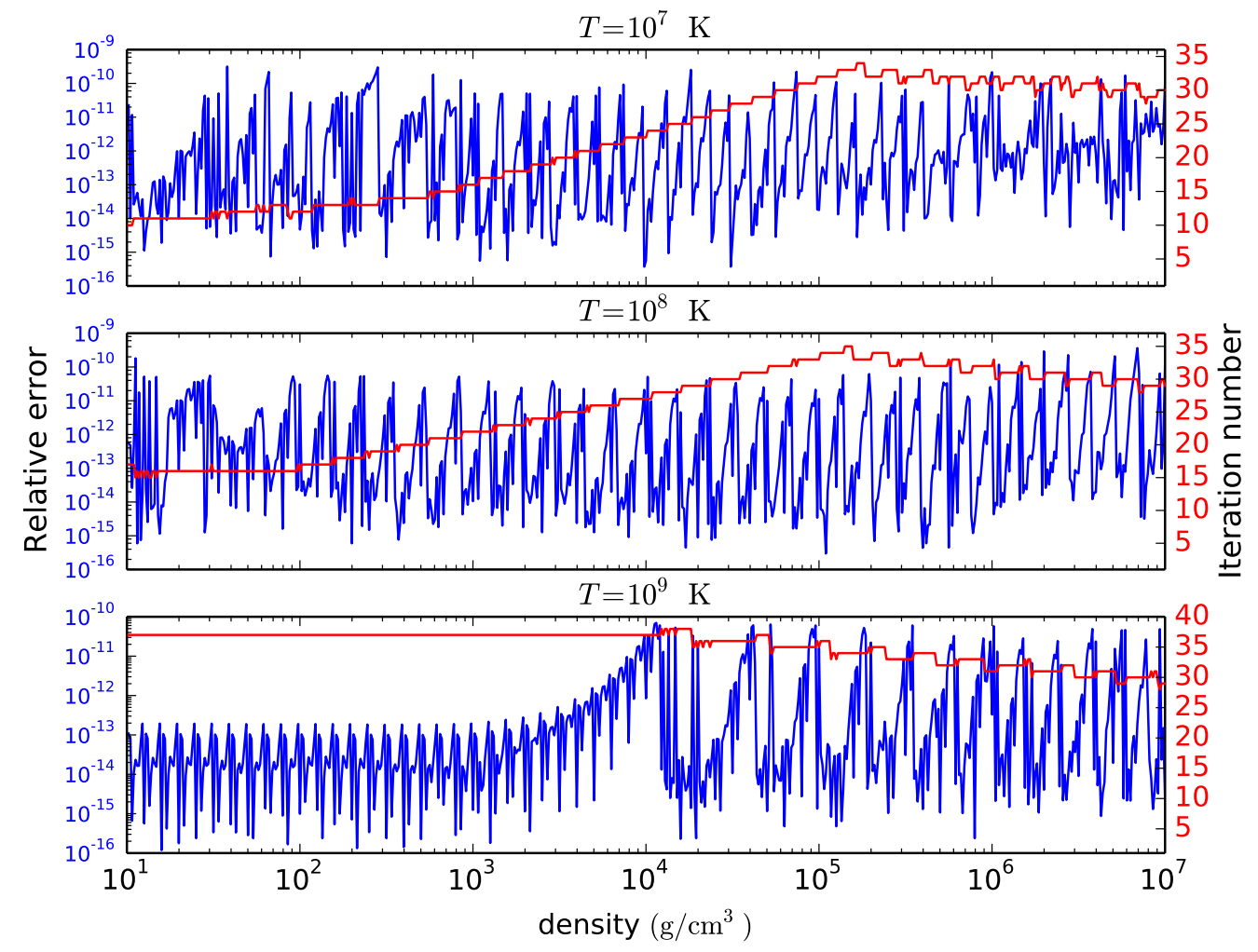

Figure 3.8: Relative errors of $(\rho, T)$ given $(P, s)$ and iterations number.

in Figure 3.8 and $(P, h)$ in Figure 3.9 , but with the relative error as the maximum one of the pair. The initial guess of $T$ and $\rho$ is chosen outside the rectangular table of the figure to demonstrate the global convergence for a bad initial guess. The first three cases Figures 3.6 to 3.8 shows overall good performance: errors below $10^{-8}$ (even below $10^{-16}$ at the discontinuing points), and number of iterations within 20. Each iteration costs the standard computing time of a call to the Helmholtz EOS. For $(P, s)$ pairs, it takes more than 30 iterations for more than half of the table owing to the complexity of $2 \mathrm{D}$ solvers. For $(P, h)$ pairs, global convergence fails often, which indicates that a good initial guess is required for the validity of the solver. Empirically speaking, a guess within $15 \%$ guarantees an efficient convergence as shown in Figure 3.9. This also implies that a good guess can reduce the number of iterations in $(P, s)$ pairs greatly. Generally speaking, the relative error reduces when the number of iterations increases 


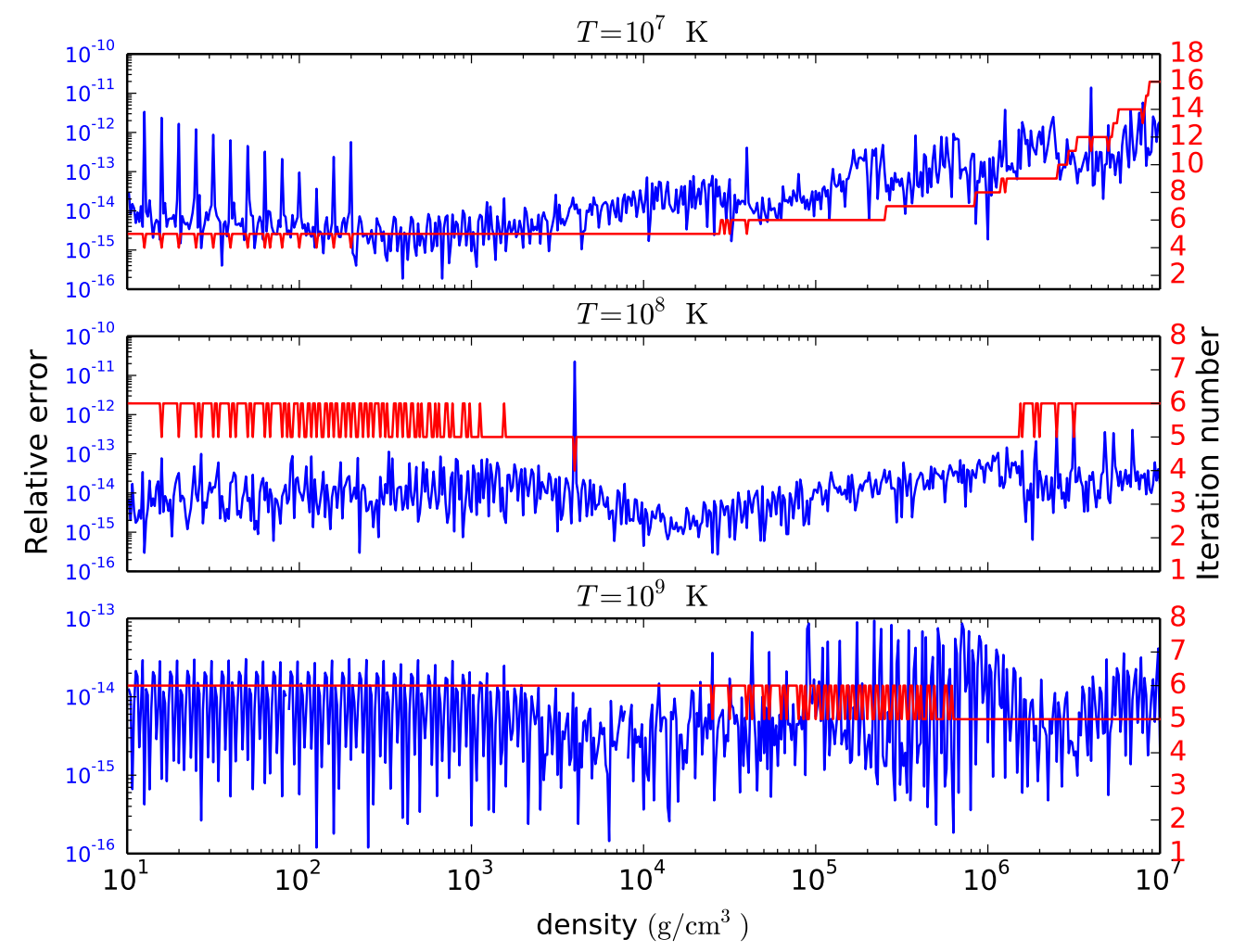

Figure 3.9: Relative errors of $(\rho, T)$ given $(P, h)$ and iterations number.

regardless of the specific structure of the function. This anticorrelation can be observed in these figures, especially in the $1 \mathrm{D}$ cases.

\subsection{Diffusion}

As mentioned in Section 3.3, there are three microscopic diffusion processes included in ODT in this dissertation: thermal diffusion, molar diffusion (or mass diffusion), and momentum diffusion. Their corresponding transport properties are the thermal diffusivity $\kappa_{T}$, molar diffusivity $\kappa_{Y}$, and momentum diffusivity (or kinematic viscosity) $\nu$, respectively. On a coordinate of column depth $m$, the diffusion equation has the form as

$$
\frac{\partial X}{\partial t}=\rho \frac{\partial}{\partial m}\left(\rho \kappa \frac{\partial X}{\partial m}\right)
$$


where $X$ is the diffusing property, and $\kappa$ is the corresponding diffusivity. There are two numerical solvers implemented in ODT for this equation, and they are discussed in detail in this section. First, it is helpful to explain the notation of variables on a computer grid. For any variable $X, X_{j}^{i}$ is a zone-centered value in zone number $j$ at time step $i, X_{j-}$ is the average of $X_{j}$ and $X_{j-1}$ that represents the value at the interface of Zone $j$ and $j-1$, and $X_{j+}$ is the average of $X_{j}$ and $X_{j+1}$ that represents the value at the interface of Zone $j$ and $j+1$. The averaging method is usually the arithmetic mean. $\mathrm{d} X$ represents the spacing difference of $X$ (or $\Delta X)$.

The first solver uses simple explicit time integration with forward differences. Set all the quantities on the RHS at time step $i$, then Equation $(3.42)$ can be written as

$$
\frac{\mathrm{d} X_{j}}{\mathrm{~d} t}=\frac{\rho_{j}^{i}}{\mathrm{~d} m_{j}}\left[\left(\rho \kappa \frac{\mathrm{d} X}{\mathrm{~d} m}\right)_{j+}-\left(\rho \kappa \frac{\mathrm{d} X}{\mathrm{~d} m}\right)_{j-}\right]^{i} .
$$

Set the difference on the LHS as a difference with respect to time, then

$$
\frac{X_{j}^{i+1}-X_{j}^{i}}{\mathrm{~d} t}=\frac{\rho_{j}^{i}}{\mathrm{~d} m_{j}}\left[\left(\frac{\rho \kappa}{\mathrm{d} m}\right)_{j+}\left(X_{j+1}-X_{j}\right)-\left(\frac{\rho \kappa}{\mathrm{d} m}\right)_{j-}\left(X_{j}-X_{j-1}\right)\right]^{i} .
$$

Solving for $X_{j}^{i+1}$, we have

$$
X_{j}^{i+1}=X_{j}^{i}+\frac{\rho_{j}^{i} \mathrm{~d} t}{\mathrm{~d} m_{j}}\left[\left(\frac{\rho \kappa}{\mathrm{d} m}\right)_{j+}\left(X_{j+1}-X_{j}\right)-\left(\frac{\rho \kappa}{\mathrm{d} m}\right)_{j-}\left(X_{j}-X_{j-1}\right)\right]^{i} .
$$

In this way, all the $X_{j}$ 's at the next time step can be obtained by known quantities at the current time step. This method is straightforward and accurate. One drawback is that the time step is constrained by the Courant-Friedrichs-Lewy (CFL) time (Courant et al. 1928)

$$
t_{\mathrm{cfl}}=\frac{\mathrm{d} m^{2}}{2\left(\kappa \rho^{2}\right)_{\max }} .
$$

Here the subscript 'max' indicates the maximum of $\kappa$ and $\rho$ of all three diffusion processes. This time parameter sets the maximum time step so that the information has enough time to propagate through grids. If the time step goes beyond $t_{\mathrm{cfl}}$, the solver will yield incorrect solutions and even crash the code. This solver has been used to resolve diffusion processes in the RTI simulation (Section 3.3) because $t_{\mathrm{cfl}}$ is in an acceptable range. In the stellar environment, $\kappa$, especially in the case of thermal diffusivity, is 
too big to hold this condition, which makes this method way too expensive to use. So another efficient solver with decent accuracy is in demand.

The second solver uses implicit time integration with forward differences. It was adopted from KEPLER code (Weaver et al. 1978a) and it has been modified to cope with more boundary conditions. For the stellar problems, thermal diffusivity is heavily emphasized because it is usually many orders of magnitude higher than the other two. So I use thermal diffusion as an example to explain this solver.

At the interfaces of Zone $j$, two flux terms are

$$
F_{j-}=-(\rho k)_{j-} \frac{T_{j-1}-T_{j}}{\mathrm{~d} m_{j-}},
$$

and

$$
F_{j+}=-(\rho k)_{j+} \frac{T_{j}-T_{j+1}}{\mathrm{~d} m_{j+}},
$$

where $k$ is the thermal conductivity. According to the energy conservation, the change of temperature is related to the total flux by

$$
\frac{\partial T_{j}}{\partial t}=\frac{1}{\left(c_{p} \mathrm{~d} m\right)_{j}}\left(F_{j+}-F_{j-}\right),
$$

where $c_{p}$ is the specific heat capacity at constant pressure. It is chosen over the heat capacity at constant volume because of Equation (3.15). For the time step, the implicit solver sets the LHS of Equation (3.49) the same as Equation (3.44), but sets the terms on the RHS at time step $i+1$ instead, namely,

$$
\frac{T_{j}^{i+1}-T_{j}^{i}}{\mathrm{~d} t}=\left[\frac{1}{\left(c_{p} \mathrm{~d} m\right)_{j}}\left(F_{j+}-F_{j-}\right)\right]^{i+1} .
$$

Combining Equations (3.47) and (3.48), it can be written as

$$
\frac{T_{j}^{i+1}-T_{j}^{i}}{\mathrm{~d} t}=\left[\frac{(\rho k)_{j+}}{\left(c_{p} \mathrm{~d} m\right)_{j} \mathrm{~d} m_{j+}}\left(T_{j+1}-T_{j}\right)-\frac{(\rho k)_{j-}}{\left(c_{p} \mathrm{~d} m\right)_{j} \mathrm{~d} m_{j-}}\left(T_{j}-T_{j-1}\right)\right]^{i+1} .
$$

It is worth noting that this equation can be simplified to Equation (3.44) by using the definition

$$
\kappa_{T} \equiv \frac{k}{c_{p} \rho}
$$


approximations $\left(c_{p}\right)_{j \pm} \approx\left(c_{p}\right)_{j}$ and $\rho_{j \pm} \approx \rho_{j}$. To solve this equation, first define

$$
\begin{aligned}
& \alpha_{j}=\left[\frac{(\rho k)_{j-}}{\left(c_{p} \mathrm{~d} m\right)_{j} \mathrm{~d} m_{j-}} \mathrm{d} t\right]^{i+1}, \\
& \gamma_{j}=\left[\frac{(\rho k)_{j+}}{\left(c_{p} \mathrm{~d} m\right)_{j} \mathrm{~d} m_{j+}} \mathrm{d} t\right]^{i+1},
\end{aligned}
$$

and

$$
\beta_{j}=1+\alpha_{j}+\gamma_{j}
$$

then Equation (3.51) can be written as

$T_{j}^{i+1}-T_{j}^{i}=\gamma_{j}\left(T_{j+1}^{i+1}-T_{j}^{i+1}\right)-\alpha_{j}\left(T_{j}^{i+1}-T_{j-1}^{i+1}\right)=\alpha_{j} T_{j-1}^{i+1}-\left(\alpha_{j}+\gamma_{j}\right) T_{j}^{i+1}+\gamma_{j} T_{j+1}^{i+1}$.

Sorting it by time step, we have

$$
T_{j}^{i}=-\alpha_{j} T_{j-1}^{i+1}+\beta_{j} T_{j}^{i+1}-\gamma_{j} T_{j+1}^{i+1}
$$

If the simulation grid (marked from number 1 to $N$ ) is isolated, there is no heat flux across boundaries, namely, $F_{1-}=0$ and $F_{N+}=0$. Then $\alpha_{1}=0, \beta_{1}=1+\gamma_{1}$, and $\gamma_{N}=0$. So Equation 3.57 has a nice matrix form,

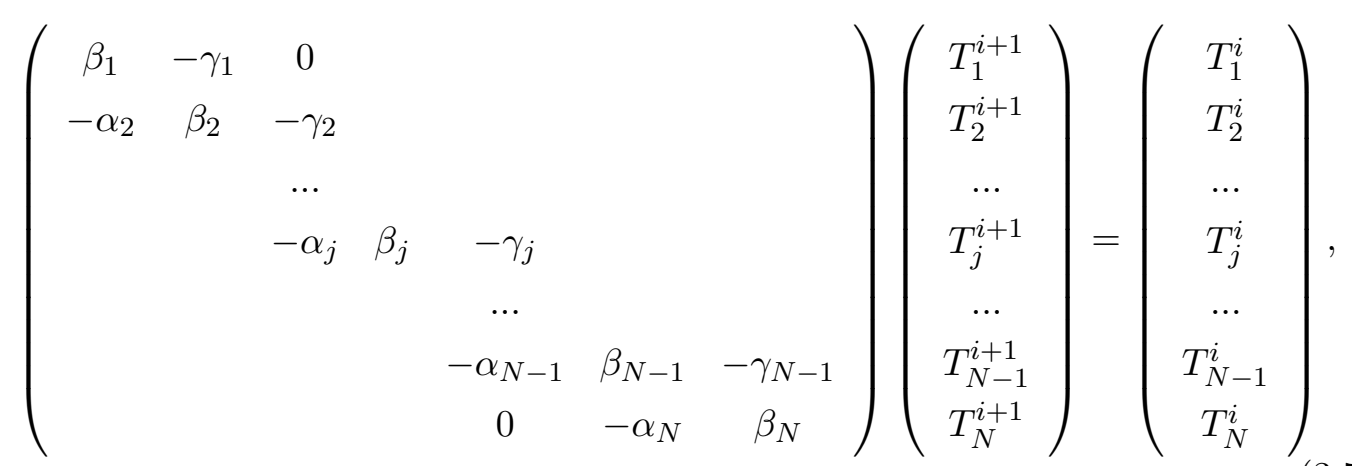

and it can be solved by Gaussian elimination (Press et al. 1993) in the following way. 
First, eliminate the $\alpha$ terms, then

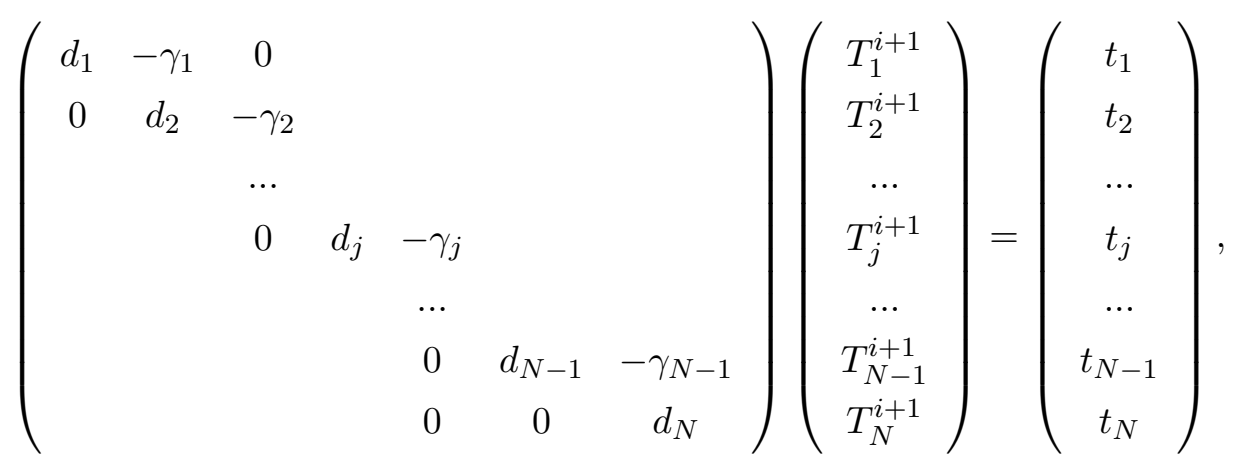

where $d_{j}=\beta_{j}-\gamma_{j-1} \frac{\alpha_{j}}{d_{j-1}}, d_{1}=\beta_{1}$, and $t_{j}=T_{j}^{i}+\frac{\alpha_{j}}{d_{j-1}} t_{j-1}, t_{1}=T_{1}^{i}$. Divide $d$ terms on both sides and set $e_{j}=\frac{1}{d_{j}}$, then

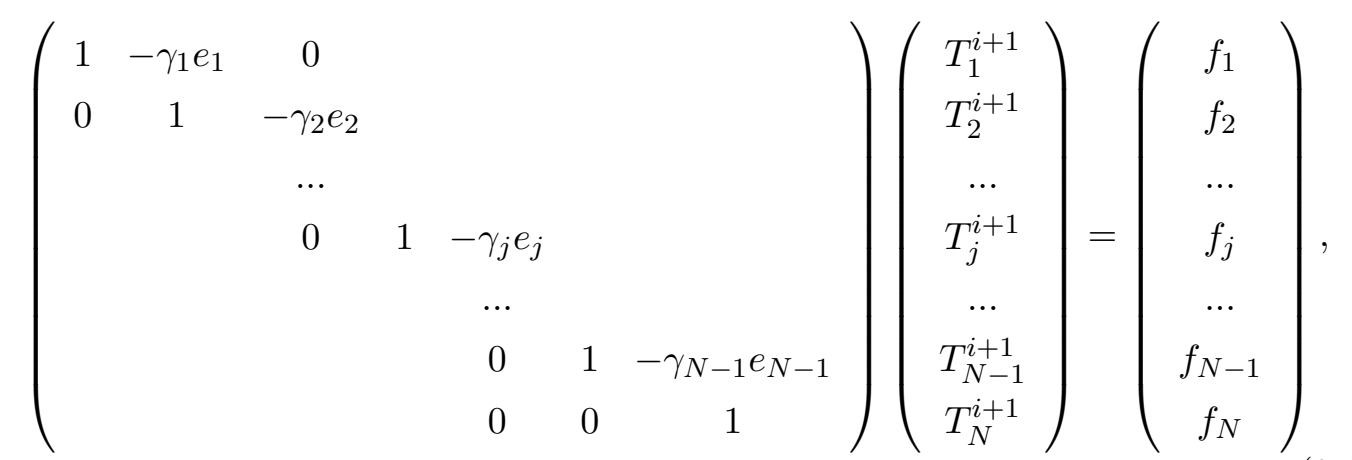

where $f_{j}=t_{j} e_{j}$. Define $h_{j}=\gamma_{j} e_{j}$, then

$$
\left(\begin{array}{ccccccc}
1 & -h_{1} & 0 & & & & \\
0 & 1 & -h_{2} & & & & \\
& & \cdots & & & & \\
& & 0 & 1 & -h_{j} & & \\
& & & & \cdots & & \\
& & & & 0 & 1 & -h_{N-1} \\
& & & & 0 & 0 & 1
\end{array}\right)\left(\begin{array}{c}
T_{1}^{i+1} \\
T_{2}^{i+1} \\
\cdots \\
T_{j}^{i+1} \\
\cdots \\
T_{N-1}^{i+1} \\
T_{N}^{i+1}
\end{array}\right)=\left(\begin{array}{c}
f_{1} \\
f_{2} \\
\cdots \\
f_{j} \\
\cdots \\
f_{N-1} \\
f_{N}
\end{array}\right) .
$$

Finally, the solution is given by

$$
T_{j}^{i+1}=h_{j} T_{j+1}^{i+1}+f_{j}
$$


with $T_{N}^{i+1}=f_{N}$.

The solution above is obtained for an isolated grid, which is usually unphysical for a star. At the top boundary, a simple atmosphere model that sets $T_{0}=0$ has the same matrix form as Equation (3.58) by using $\alpha_{1} \neq 0$ and $\beta_{1}=1+\alpha_{1}+\gamma_{1}$. At the bottom boundary, consider a constant flux $F_{N+}=F_{b}$ in Equation (3.51),

$$
\frac{T_{N}^{i+1}-T_{N}^{i}}{\mathrm{~d} t}=\frac{1}{\left(c_{p} \mathrm{~d} m\right)_{N}}\left[F_{b}-F_{N-}\right]^{i+1}=\left[\frac{F_{b}}{\left(c_{p} \mathrm{~d} m\right)_{N}}-\frac{\alpha_{N}}{\mathrm{~d} t}\left(T_{N}-T_{N-1}\right)\right]^{i+1} .
$$

Define

$$
T_{b}=\left[\frac{F_{b} A_{b}}{\left(c_{p} \mathrm{~d} m\right)_{N}} \mathrm{~d} t\right]^{i+1},
$$

then the matrix form is retained by replacing $T_{N}^{i}$ by $T_{N}^{i}+T_{b}$,

$$
T_{N}^{i}+T_{b}=-\alpha_{N} T_{N-1}^{i+1}+\left(1+\alpha_{N}\right) T_{N}^{i+1}=-\alpha_{N} T_{N-1}^{i+1}+\beta_{N} T_{N}^{i+1} .
$$

To compare these two solvers, a simple test that diffuses a step-function profile without diffusion across the boundary is discussed in this paragraph. The initial configuration is set up by filling half of an isolated grid with diffusing property, i.e., temperature, molar fraction, or velocity, leaving the other half of the grid empty. The grid spans from 0 to 1 with a uniform resolution equal to 0.01 . The transport properties and densities are set to unity for simplicity. For the explicit solver, the time step is set to the CFL time (Equation 3.46), and the number of time steps is set to 5000. For the implicit solver, the test runs five cases, in which the time steps increase by a factor of $1,10,100$, 1000, and 5000, respectively. The results are compared at the same ending time, so the numbers of time steps are 5000,500,50,5, and 1, respectively. The absolute values of relative errors are shown in Figure 3.10. When the time step is comparable (blue and red curves), the results of these two methods match well with the biggest relative error around $10^{-3}$. As the time step increases, the biggest relative error increases from $10^{-3}$ to 0.2 . Even when the time step increases by 5000 and only one time step is calculated (the black curve), the implicit solver is still stable. All the curves show symmetry with respect to a line at the mass equal to 0.5. This is because the symmetry is, in fact, with respect to the center point of each curve and the relative errors has been taken absolute values. Both solvers demonstrate good conservation of the diffusing property with a relative error less than $10^{-13}$. 


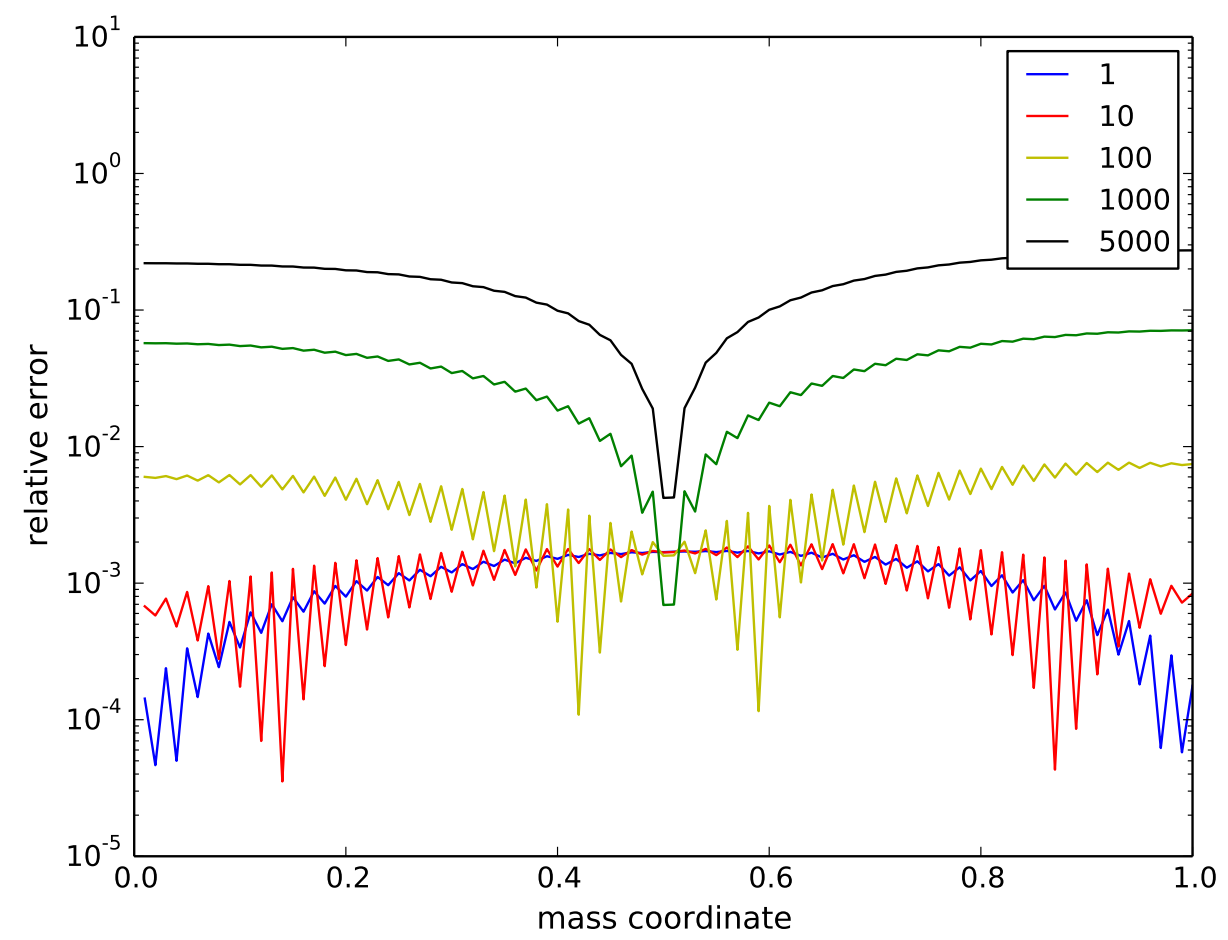

Figure 3.10: Comparison of two diffusion solvers. The test is set up with a step-function profile diffusing on an isolated grid. The time step is set as the CFL time for the explicit solver, while the time steps are varied by five different factors $(1,10,100,1000$, and $5000)$ for the implicit solver. The relative errors are calculated at the same ending time for both solvers, which indicates that the numbers of steps for the implicit solver are $5000,500,50,5$, and 1 , respectively.

After a discussion of the numerical solvers, I briefly introduce the transport properties used in ODT in the rest of this section with a focus on the thermal diffusivity owing to its dominant magnitude. The routine is adopted from Frank Timmes' work (Timmes 2000. In detail, the thermal conductivity is calculated in two parts,

$$
k=k_{\mathrm{rad}}+k_{\mathrm{cond}}=\frac{4 a c T^{3}}{\rho}\left(\frac{1}{\kappa_{\mathrm{rad}}}+\frac{1}{\kappa_{\text {cond }}}\right),
$$

where $c$ is the speed of light, $a$ is a constant and $a=4 \sigma / c, \kappa_{\text {rad }}$ and $\kappa_{\text {cond }}$ are the radiative and conductive opacities, respectively. In the case of radiative opacity, Iben (1975) and Christy (1966) are used for all processes other than electron scattering. Weaver et al. 
(1978b) is used for the Compton opacity, which includes a cutoff for frequencies less than the plasma frequency. The conductive opacity is calculated by the following two parts. In the non-degenerate regime, analytic fits from Iben (1975) are used, whereas in the degenerate regime, Yakovlev \& Urpin (1980) is used.

\subsection{A simple burst model with three isotopes}

After a long run of code building, ODT is finally ready for a simple XRB model with a basic 3-isotope burning process. This model is designated to investigate a hypothesis Alexander Heger had in his KEPLER models for hydrogen-helium bursts (Woosley et al. 2004). The general picture is that after a layer of hydrogen and helium accreted, carbon are produced through triple- $\alpha$ reactions. Because of the presence of hydrogen, carbon is quickly burned into nitrogen in the CNO cycle. Thus a layer of nitrogen can be formed for a brief time separating the hydrogen and newly produced carbon. This separation can lead to an instability due to the heat accumulation in the carbon layer below. If the energy of turnover is big enough to penetrate the nitrogen layer, the mixing of carbon and hydrogen layers generates a lot of energy that is enough to power the subsequent reactions and eventually trigger an XRB.

The environment of the model is a thin shell $(\sim 10 \mathrm{~m})$ of accreted material on the surface of a neutron star of a typical $1.4 M_{\odot}$ and $10^{6} \mathrm{~cm}$ radius. So the plane parallel assumption is valid. The initial set-up is shown in Figure 3.11. The simulation domain is divided into two parts (indicated by the vertical dashed line) which are filled by hydrogen and carbon, respectively. The mass fraction is represented by the blue axis on the left. An error function is applied to smooth the abundance profiles near the separation line in order to provide the initial mixing and avoid discontinuity of other profiles. The hydrogen part is arranged with a very small domain so that the mixing near the surface of the star can be examined. Since the mixing is hopefully triggered by the heat generated from the nuclear burning, density and temperature profiles are set to a stable configuration in terms of entropy. The logarithmic axis for these quantities is on the right in the red color. In detail, a temperature $T=5.83 \times 10^{8} \mathrm{~K}$ is set at depth $m=8.18 \times 10^{7} \mathrm{~g} / \mathrm{cm}^{2}$. Both of the values are taken from a KEPLER model. According to Equation (3.15), pressure can be obtained from the column depth and the physical 


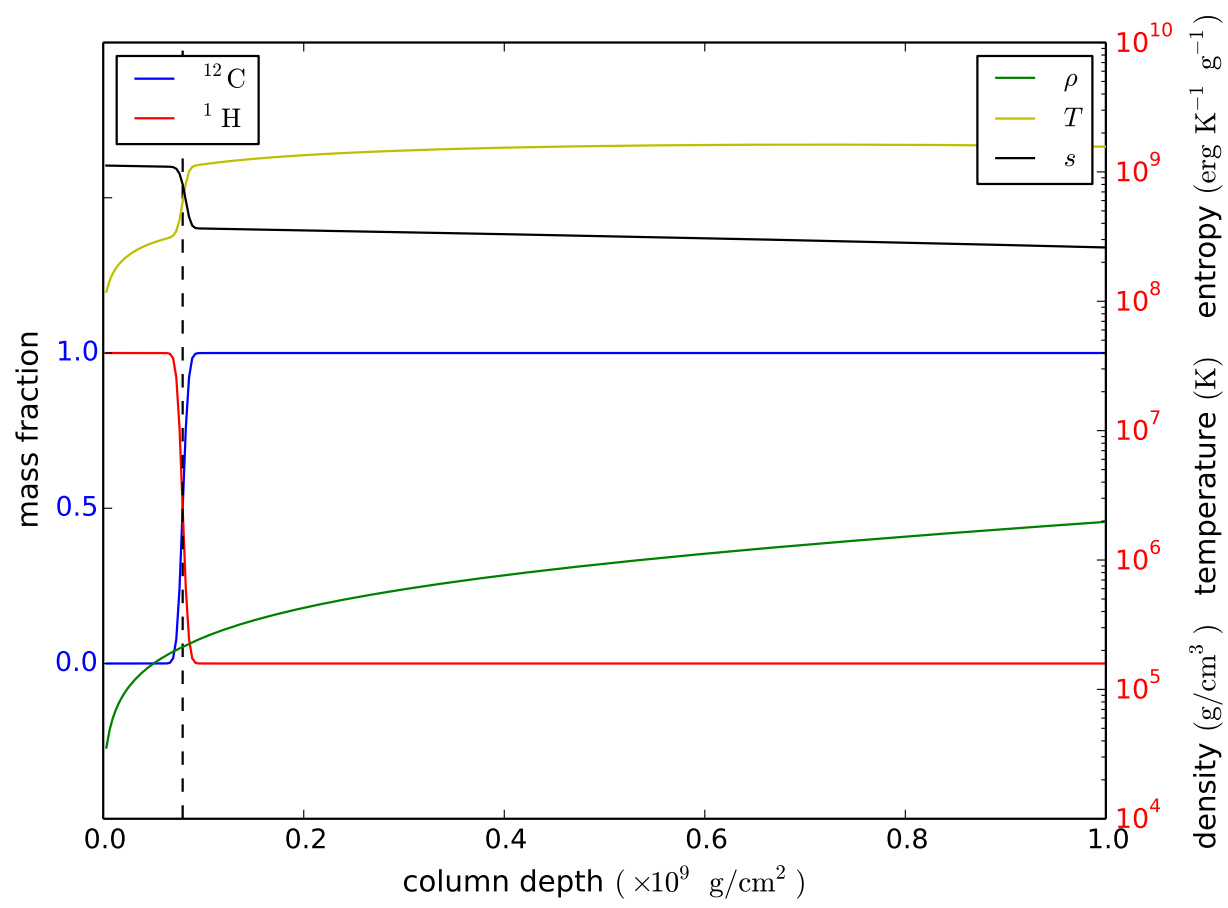

Figure 3.11: Initial set-up of the 3-isotope model. The abundance of hydrogen and carbon is associated to the left blue axis, while the density, temperature, and specific entropy are associated to the right red axis. A stable configuration in terms of entropy is set up by varying entropy with a factor determined by the distance between a grid point and a reference point (indicated by the vertical dashed line). An error function is applied to smooth the profiles near the interface and avoid any discontinuity in the properties.

state at this depth can be determined by the EOS in the case of $(P, T)$ pair. Here the temperature is chosen prior to the density, because the nuclear reaction rate is more sensitive to temperature. The EOS yields the specific entropy at this depth, then an entropy reference point is obtained and we build a stable entropy profile around it. If a grid point at a different depth moves quickly to this point, the materials inside go through an adiabatic process and end up with the same pressure. Assume the density is close to that of the reference point too, the EOS yields the entropy for this grid point in the case of $(P, \rho)$ pair. Reverse the previous adiabatic process, the physical state of the grid point at its original depth is also determined in the case of $(P, s)$ pair. Vary this 
entropy by a percentage factor with respect to its distance from the reference point, so a stable configuration with decreasing entropy gradient is obtained. The other profiles can be calculated by the EOS with the new entropy and known pressure. Multiple tests have been carried out with the percentage factor ranging from a few percent to $50 \%$. And the most stable case $(50 \%)$ is shown in Figure 3.11

Due to the limited nuclear networks, helium is not included in the model. Hence there is only one reaction involved: ${ }^{12} \mathrm{C}(p, \gamma){ }^{13} \mathrm{~N}$. The differential equations of abundance are

$$
\frac{\mathrm{d} Y_{\mathrm{H}}}{\mathrm{d} t}=-\lambda Y_{\mathrm{H}} Y_{\mathrm{C}}
$$

and

$$
\frac{\mathrm{d} Y_{\mathrm{C}}}{\mathrm{d} t}=-\lambda Y_{\mathrm{H}} Y_{\mathrm{C}}
$$

where $Y$ is again the molar fraction, and $\lambda$ is the reaction rate from Caughlan \& Fowler (1988). Because of the mass conservation,

$$
Y_{\mathrm{C}}+Y_{\mathrm{H}}=1,
$$

the abundance can be solved analytically.

The difficulty, however, lies in the small molecular diffusivity. At the nuclear burning time scale, the mixing due to microscopic molecular diffusion is too small to be considered, and the assumed adiabatic mappings don't allow any mixing between zones. It results in isolated zones for a uniform grid. In other words, the fuels burn out in the mixed zones and the abundance stays the same thereafter. Theoretically speaking, it is possible to resolve this issue by switching to the adaptive-mesh method for the implementation of a map in which the mixing can be achieved by zone splitting and merging. Such mixing, however, would be purely numerical and hence be artificial. A compromising solution is adding a subgrid model to support the triplet maps on turbulent mixing. And the mechanism from Clement (1993) is adopted. The general idea is to model the turbulent viscosity as

$$
\nu_{\mathrm{turb}}=\omega \lambda_{\mathrm{G}} q^{1 / 2}
$$

where $\omega$ is a dimensionless parameter of order unity, $\lambda_{\mathrm{G}}$ is the grid scale, and $q$ is the turbulent kinetic energy. Then the other two turbulent transport properties can be 


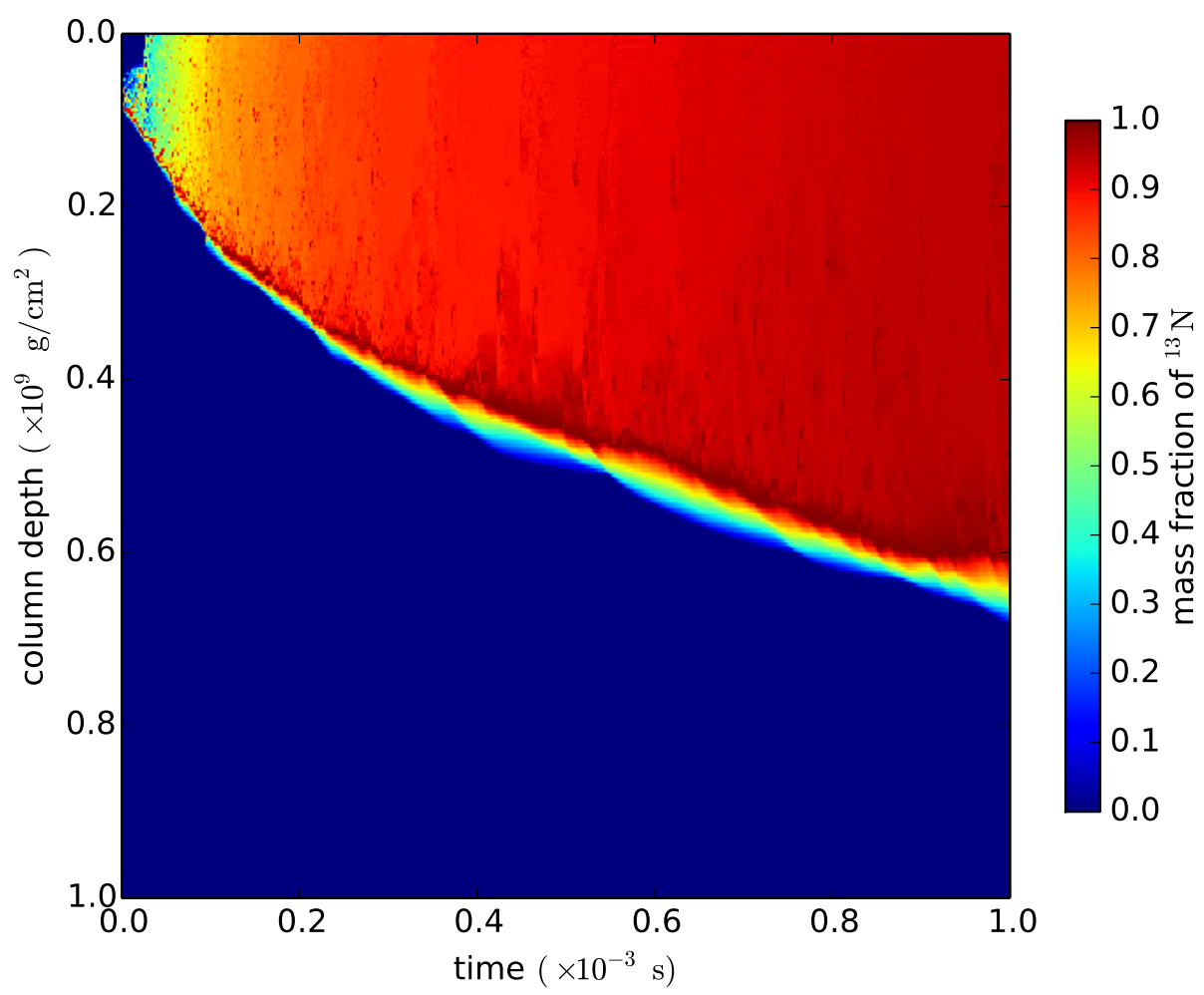

Figure 3.12: Evolution of the nitrogen mass fraction profile. The color represents the mass fraction. It is the result of a single realization calculation with $C=1.0$ and $Z=0$. The viscous penalty term is ignored since the viscosity is comparatively small in the environment.

obtained by

$$
\kappa_{T}=\frac{\nu}{P_{r}}
$$

and

$$
\kappa_{Y}=\frac{\nu}{S_{c}}
$$

Here $P_{r}$ and $S_{c}$ are the turbulent Prandtl and Schmidt numbers, respectively. Both of them are assumed to be $1 / 3$. The Richardson's criterion is applied to switch the subgrid model on and off. The definition of the Richardson number at the zone interface is

$$
R_{i}=\frac{N^{2}}{\left(\frac{\mathrm{d} u}{\mathrm{~d} z}\right)^{2}},
$$




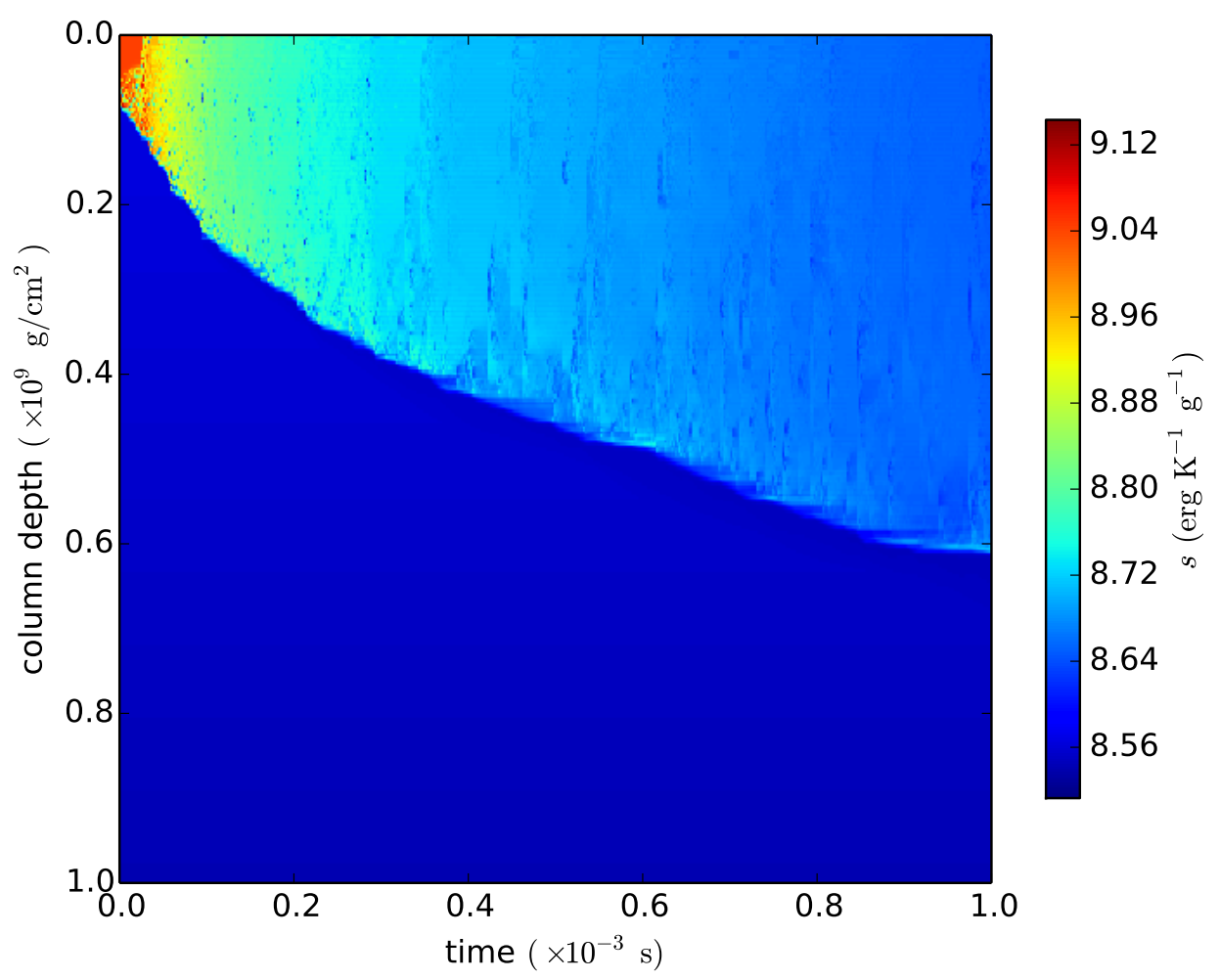

Figure 3.13: Evolution of the specific entropy $(s)$ profile. The color represents the logarithmic value of the specific entropy. It is the result of a single realization calculation with $C=1.0$ and $Z=0$.

where $N$ is the Brunt-Väisälä frequency, $u$ is the velocity, and $z$ is the spatial distance. The critical value of $R_{i}$ is approximately $1 / 4$ (Miles 1961). If $R_{i} \geq 1 / 4$, the system is considered stable; If $R_{i}<1 / 4$, diffusion with turbulent diffusivities is allowed across the zone interface.

The results of a single realization with a parameter set $(C, Z)=(1.0,0)$ are shown in Figures 3.12 to 3.14 . Here $Z=0$ is because the viscous effect is negligible compared with the other energy terms. Eddy events initiate shortly after the simulation starts around the reference point as the stable entropy gradient is reserved in the mixed fuel region (see Figure 3.13). Then the mixing region quickly expands in both directions and a layer of nitrogen starts to appear as expected (see Figure 3.12). But the layer is 


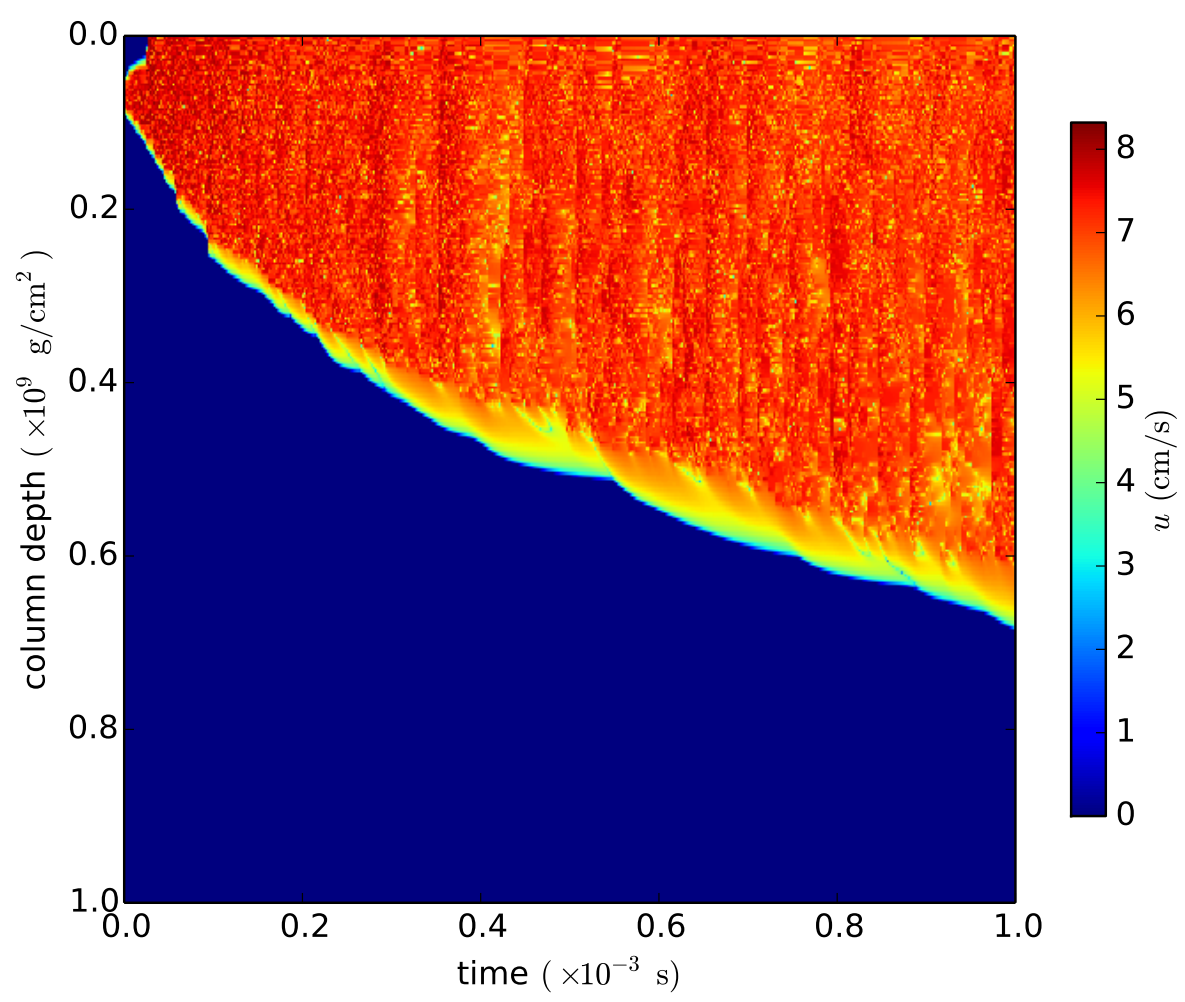

Figure 3.14: Evolution of the velocity $(u)$ profile. The color represents the logarithmic value of the velocity. It is the result of a single realization calculation with $C=1.0$ and $Z=0$. Isotropic assumption is applied.

not restricted to a narrow band. The hydrogen left in the mixing region keeps diffusing to lower carbon layer, which causes the nitrogen layer continuously eating the carbon layer. From Figure 3.14, it is clear that the diffusing front is powered by the turbulent diffusion introduced by the subgrid model instead of eddy events. In other words, the Richardson number in this region is small owing to the large velocity gradients. This process decays as hydrogen depletes and the energy dissipates to larger area. Different set-ups have been carried out, e.g., various stable entropy configurations (by changing the percentage factors), various initial mixing regions (by changing the widths of the error function), and various realizations (by changing the sequences of random numbers), but this feature of expanding nitrogen layer remains the same. As a result, the layer 
of nitrogen doesn't separate the hydrogen and carbon layers completely but expands according to the Richardson's criterion. The energy cannot build up in an explosive way but increases gradually as the layer expands. Although the result is not completely as predicted, the test shows that ODT is ready for XRBs simulations once a nuclear network is implemented. 


\section{Chapter 4}

\section{Pure Helium Bursts Simulations Using ODT}

In this chapter, I present the pure helium bursts simulations. I start with an introduction to a nuclear network that approximates the $\alpha$-chain reactions using 19 isotopes. Then I show the initial configuration and formulation of the ODT models. A test of the nuclear burning process without any turbulent motion is discussed next to check all the pieces of physics included except the mixing mechanisms. The rest of the chapter focuses on the comparison and discussion of simulation results such as the light curves, turbulent motions, and abundance with a particular focus on the turbulent motions caused by different mixing mechanisms.

\subsection{Nuclear network}

Theoretically speaking, PRE bursts are usually caused by pure helium bursts or hydrogen-poor bursts. There are two reasons for the neutron star atmosphere to be hydrogen-poor. First, the hydrogen-rich layer above is ejected during the bright burst (Sugimoto et al. 1984, Ebisuzaki \& Nakamura 1988). Second, the mass donor is a helium-rich white dwarf. A typical example is the ultracompact binary system $4 \mathrm{U}$ 1820-30 which has a short orbital period of 11.4 minutes (Stella et al. 1987).

A 19-isotope nuclear network that focuses on the $\alpha$-chain reactions is included in ODT for the application of simulating PRE bursts. The subroutine is adopted from 


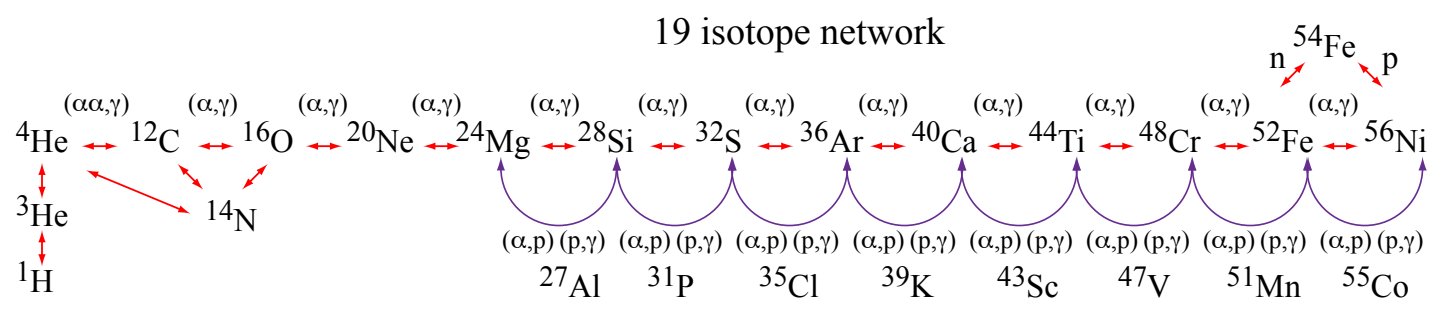

Figure 4.1: 19-isotope nuclear network. The explicit 19 isotopes are connected by the red arrows while the implicit 8 isotopes are connected by the blue arrows. The latter are included by assuming that the abundance is in steady state with the neighboring nuclei. The protons in the upper right corner come from photodisintegration. Courtesy of Frank Timmes.

Frank Timmes' website (Timmes 2015a) and the details are described in Weaver et al. (1978b). The 19 isotopes covered in this network $\left({ }^{1} \mathrm{H},{ }^{3} \mathrm{He},{ }^{4} \mathrm{He},{ }^{12} \mathrm{C},{ }^{14} \mathrm{~N},{ }^{16} \mathrm{O},{ }^{20} \mathrm{Ne}\right.$, ${ }^{24} \mathrm{Mg},{ }^{28} \mathrm{Si},{ }^{32} \mathrm{~S},{ }^{36} \mathrm{Ar},{ }^{40} \mathrm{Ca},{ }^{44} \mathrm{Ti},{ }^{48} \mathrm{Cr},{ }^{52} \mathrm{Fe},{ }^{54} \mathrm{Fe},{ }^{56} \mathrm{Ni}$, protons from photodisintegration, and neutrons) are shown in Figure 4.1 and the reactions are indicated by the red arrows. 8 isotopes connected by the blue arrows $\left({ }^{27} \mathrm{Al},{ }^{31} \mathrm{P},{ }^{35} \mathrm{Cl},{ }^{39} \mathrm{~K},{ }^{43} \mathrm{Sc},{ }^{47} \mathrm{~V},{ }^{51} \mathrm{Mn}\right.$, and ${ }^{55} \mathrm{Co}$ ) are included implicitly by assuming that the abundance is in steady state with neighboring nuclei.

The general idea of this nuclear network is described as follows (see Timmes 1999). On a Lagrangian grid, the general continuity equation for isotope $i$ is

$$
\frac{\mathrm{d} Y_{i}}{\mathrm{~d} t}+\nabla \cdot\left(Y_{i} \mathbf{V}_{\mathbf{i}}\right)=\dot{R}_{i}
$$

where $Y_{i}=X_{i} / A_{i}$ is the molar abundance, $V_{i}$ is the mass diffusion velocity, and $\dot{R}_{i}$ is the total reaction rate. For binary reactions of the form $i(j, k) l$, the reaction rates can be written as

$$
\dot{R}_{i}=\sum_{j, k} Y_{l} Y_{k} \lambda_{k j}(l)-Y_{i} Y_{j} \lambda_{j k}(i)
$$

with $\lambda_{k j}$ and $\lambda_{j k}$ being the reverse and forward reaction rates respectively. And they are related to the relative velocity $v$ and reaction cross section $\sigma$ by

$$
\lambda_{k j}=\rho\left\langle N_{A} v \sigma\right\rangle_{k, l},
$$

and

$$
\lambda_{j k}=\rho\left\langle N_{A} v \sigma\right\rangle_{i, j}
$$


where $N_{A}$ is the Avogadro's constant. For triple- $\alpha$ reaction, some modifications need to be made for coefficients,

$$
\dot{R}_{4} \mathrm{He}=3 Y_{{ }^{12} \mathrm{C}} \lambda_{\left({ }^{12} \mathrm{C} \rightarrow{ }^{4} \mathrm{He}\right)}-\frac{3}{3 !} Y_{4}^{3} \mathrm{He} \lambda_{\left({ }^{4} \mathrm{He} \rightarrow{ }^{12} \mathrm{C}\right)} .
$$

The nuclear reaction rates $(\lambda)$ are calculated according to Woosley et al. (1975); Holmes et al. (1976); Woosley et al. (1978); Caughlan \& Fowler (1988). In a neutral plasma, nuclei are surrounded by electron clouds, which reduces the effective charges of nuclei in the Coulomb interaction and decreases the Coulomb barrier of nuclear reactions. Thus the reaction rates should be enhanced by a multiplier called the screening factor. For weak screening, the network calculates the factors based on Graboske et al. (1973). For strong screening, Alastuey \& Jancovici (1978) is used and plasma parameters are calculated based on Itoh et al. (1979).

Because the nuclear burning time scale is much shorter than that of mass diffusion, the velocity term in Equation (4.1) can be neglected. Therefore the equation is modified to the standard form

$$
\dot{Y}=\dot{R}=f(Y)
$$

which constitutes a reaction network. To solve this set of ordinary differential equations, an implicit time integration solver is preferred because it is stiff in that the abundance of at least one isotope evolves much faster than the others. Practically speaking, the variable-order Bader-Deuflhard method combined with the linear algebra package MA28 (Duff et al. 1986) are used for accuracy and overall efficiency.

\subsection{Initial configuration}

The initial configuration of our XRB simulation is linearly mapped from the top thin layer of a KEPLER model of burster 4U 1820-30 built by Laurens Keek. The environment is a thin shell of accreted material without hydrogen on the surface of a neutron star of a typical $1.4 M_{\odot}$ and $10^{6} \mathrm{~cm}$ radius. The accretion rate is $3 \times 10^{-9} M_{\odot} / \mathrm{yr}$ or $1.89 \times 10^{17} \mathrm{~g} / \mathrm{s}(10 \%$ Eddington accretion rate) and the bottom heating from the neutron star crust is set as $0.45 \mathrm{MeV}$ per nucleus. The latter is higher than $0.1-0.15 \mathrm{MeV}$ per nucleus usually taken to reduce the recurrence time to $\sim 3.5$ hours. The detailed configuration is summarized in Figure 4.2. All the prominent abundance is marked 


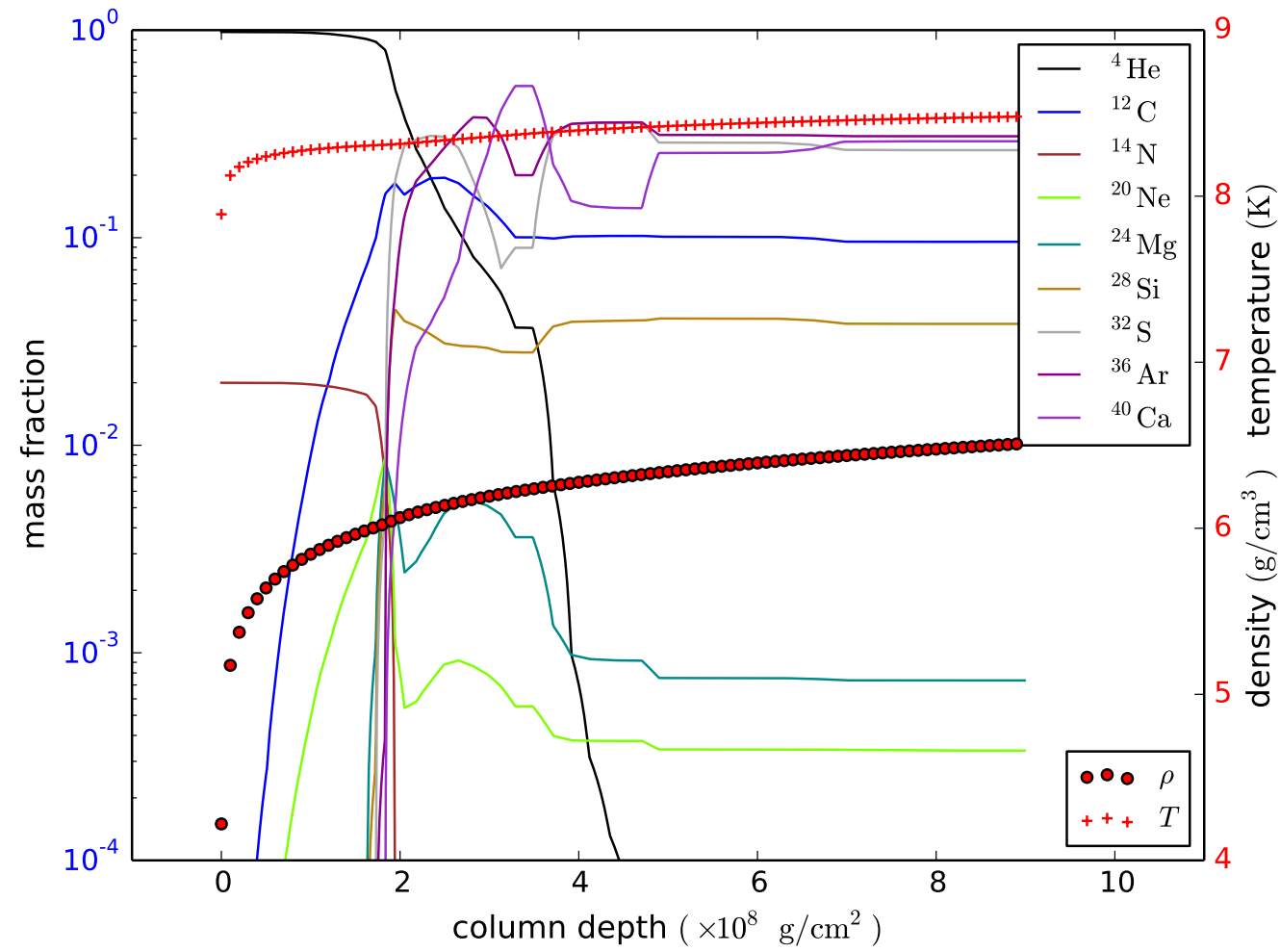

Figure 4.2: Initial set-up of the pure helium burst model. All the prominent abundance is marked by color curves and the values are shown on the left blue axis. The density and temperature are represented by red dots and crosses with their values shown on the right red axis, respectively. The simulation domain is the top thin layer of a KEPLER model built by Laurens Keek.

by color curves on the left axis on a logarithmic scale. As shown by the black curve, most of the simulation region is filled with helium. The temperature and density are marked by crosses and dots on the right axis on a logarithmic scale, respectively. The temperature is above $10^{8} \mathrm{~K}$ except around the surface region, which indicates that the triple- $\alpha$ reaction is ongoing and the heat is accumulating for the later runaway.

The simulation domain of ODT is chosen from the surface of the star to a depth at $y=9 \times 10^{9} \mathrm{~g} / \mathrm{cm}^{2}$. At the top boundary, a similar atmosphere model is applied in which the top zone is in touch with a vacuum above. A flux-limited diffusion (FLD) model (Levermore \& Pomraning 1981) is adopted to reduce the flux near the surface during 
the expansion when it becomes optically thin to avoid the causality problem (Turner \& Stone 2001). At the bottom boundary, a constant flux of $7.58 \times 10^{21} \mathrm{erg} \mathrm{s}^{-1} \mathrm{~cm}^{-2}$ is applied. This value is interpolated from the flux of the KEPLER model at the same depth. According to tests, small variation of this quantity does not change the burst structure and the light curve because the burst energy mainly comes from the nuclear reactions. But the burst initiation time is influenced by the variation because it provides the heat to trigger the runaway. Another concern of this choice of boundary condition is that it can introduce a flux feed back once the heat generated from the runaway reaches the bottom boundary. So the simulation domain is chosen large enough to eliminate this effect.

\subsection{Formulation}

The formulation of eddy sampling is similar to that in Section 3.3 . The energy conservation during an eddy trial indicates

$$
\begin{aligned}
\left(\frac{1}{2} \int \mathbf{u}_{\mathbf{f}}^{2} \mathrm{~d} m+\int g y_{f} \mathrm{~d} m+\int e_{f} \mathrm{~d} m\right) & -\left(\frac{1}{2} \int \mathbf{u}^{2} \mathrm{~d} m+\int g y \mathrm{~d} m+\int e \mathrm{~d} m\right) \\
& =\int p\left(\frac{1}{\rho_{f}}-\frac{1}{\rho}\right) \mathrm{d} m,
\end{aligned}
$$

where the three terms in the brackets on the left hand side are again the kinetic energy, potential energy, and thermal energy, respectively, the term on the right hand side is the work done to the eddy region. The only difference from Equation (3.6) is that the specific thermal energy is represented by $e$ instead as there is no analytic expression in this case. The heat generated from nuclear burning is not included in this process for simplicity and to save computing time. Combining the velocity modification

$$
\mathbf{u}_{\mathbf{f}}=\mathbf{u}+\mathbf{c} K(m)
$$

we can solve for $\mathbf{c}$ and find a same result as Equation (3.7)

$$
c_{i}=\frac{27}{4 l_{m}}\left(-u_{i, K}+\operatorname{sgn}\left(u_{i, K}\right) \sqrt{\frac{1}{3}\left(\sum_{i=1}^{3} u_{i, K}^{2}-\frac{8}{27 l_{m}} \Delta E\right)}\right),
$$

The difference is that the term $\Delta E$ has the expression

$$
\Delta E=g \int\left(y_{f}-y\right) \mathrm{d} m+\int\left(e_{f}-e\right) \mathrm{d} m-\int p\left(\frac{1}{\rho_{f}}-\frac{1}{\rho}\right) \mathrm{d} m .
$$


Because the viscous penalty is negligible, i.e., $Z=0$, the eddy rate distribution is

$$
\lambda=\frac{C}{l^{3}} \sqrt{\sum_{i=1}^{3} u_{i, K}^{2}-\frac{8}{27 l_{m}} \Delta E .}
$$

So only one free parameter $C$ is considered in this application.

In the absence of rotation and magnetic fields, the momentum conservation along the radial direction (represented by the subscript ' 2 ') indicates

$$
\frac{\mathrm{d} u_{2}}{\mathrm{~d} t}=-\frac{1}{\rho} \frac{\partial P}{\partial r}-\frac{G M}{r^{2}}+\rho \frac{\partial}{\partial m}\left(\rho \nu \frac{\partial u_{2}}{\partial m}\right) .
$$

The first two terms on the right hand side of Equation 4.12 are cancelled due to hydrostatic equilibrium condition,

$$
\frac{\partial P}{\partial r}=-\frac{G M}{r^{2}} \rho
$$

Therefore Equation 4.12 can be simplified as

$$
\frac{\partial u_{2}}{\partial t}=\rho \frac{\partial}{\partial m}\left(\rho \nu \frac{\partial u_{2}}{\partial m}\right)
$$

where the material derivative has been replaced by partial derivative because of the Lagrangian coordinate. The simplified equation has the same format as the momentum diffusion equations in the other two directions, so the momentum conservation can be summarized as

$$
\frac{\partial u_{i}}{\partial t}=\rho \frac{\partial}{\partial m}\left(\rho \nu \frac{\partial u_{i}}{\partial m}\right), i=1,2,3 .
$$

For energy conservation, we consider the change of enthalpy instead of internal energy due to the isobaric process in each cell (Equation 3.15). According to Equation (3.16), the change of enthalpy only comes from the heat term $T \mathrm{~d} s$. In other words, we can write the energy conservation equation as

$$
\frac{\mathrm{d} h}{\mathrm{~d} t}=-\frac{\partial L}{\partial m}+\epsilon
$$

where $L$ is the heat flow (luminosity), and $\epsilon$ is the local nuclear energy generation rate per unit mass. Here the viscous dissipation is neglected owing to small stellar viscosity. The heat flow is computed according to the thermal diffusion process

$$
\frac{\partial T}{\partial t}=\rho \frac{\partial}{\partial m}\left(\rho \kappa_{T} \frac{\partial T}{\partial m}\right),
$$


where $\kappa_{T}$ is the thermal diffusivity. It is the dominant diffusivity among all the diffusivities and its calculation is discussed in detail in Section 3.5. Compared with the fitting formulae used in the KEPLER model, the only difference lies in the part of conductive opacity in the degenerate regime. In ODT models, Yakovlev \& Urpin (1980) is used for calculating the conductive opacity at high density. But in the KEPLER model, Iben (1975) is used instead. Because we are more interested in the opacity near the surface where electrons are not degenerate, this difference is not playing an important role in the results. The calculation of $\epsilon$ involves another diffusion process

$$
\frac{\partial Y_{i}}{\partial t}=\rho \frac{\partial}{\partial m}\left(\rho \kappa_{Y} \frac{\partial Y_{i}}{\partial m}\right)
$$

where $Y_{i}$ is the molar fraction of isotope $i$, and $\kappa_{Y}$ is the molar diffusion coefficient. Once $\mathrm{d} h$ is obtained, the EOS can solve the state with the known pair $(h, P)$ as discussed in Section 3.5 ,

\subsection{Nuclear network comparison}

Since the nuclear reactions are crucial in the development of the burst, it is necessary to compare the two nuclear networks used in these two models first. Overall, there are two parts needed to compare in detail: the screening factors and the reaction rates. The formulae for screening factors used in the KEPLER model are exactly the same as the ones discussed in Section 4.1. And the difference of the networks comes only from the calculation of the reaction rate of ${ }^{12} \mathrm{C}(\alpha, \gamma){ }^{16} \mathrm{O}$. The original KEPELER model uses relatively new rate (Buchmann 1996) and yields a faster burning process. So the easiest way to eliminate this difference is to switch the KEPLER model to the built-in old rate for reaction ${ }^{12} \mathrm{C}(\alpha, \gamma){ }^{16} \mathrm{O}$ and modify it by a factor of 1.7 to match the one used in ODT. To test this modification, one simulation has been run for each model without any turbulent motion. In other words, turbulent motion has been isolated in this test for both models.

The comparison results are shown in Figures 4.3 to 4.5 . The colors in Figure 4.3 shows the nuclear energy generation rate per unit mass on a logarithmic scale for both models with ODT on the top and KEPLER on the bottom. The y-axis is the column depth, and the $\mathrm{x}$-axis is the time since the initial configuration. There is a time offset 


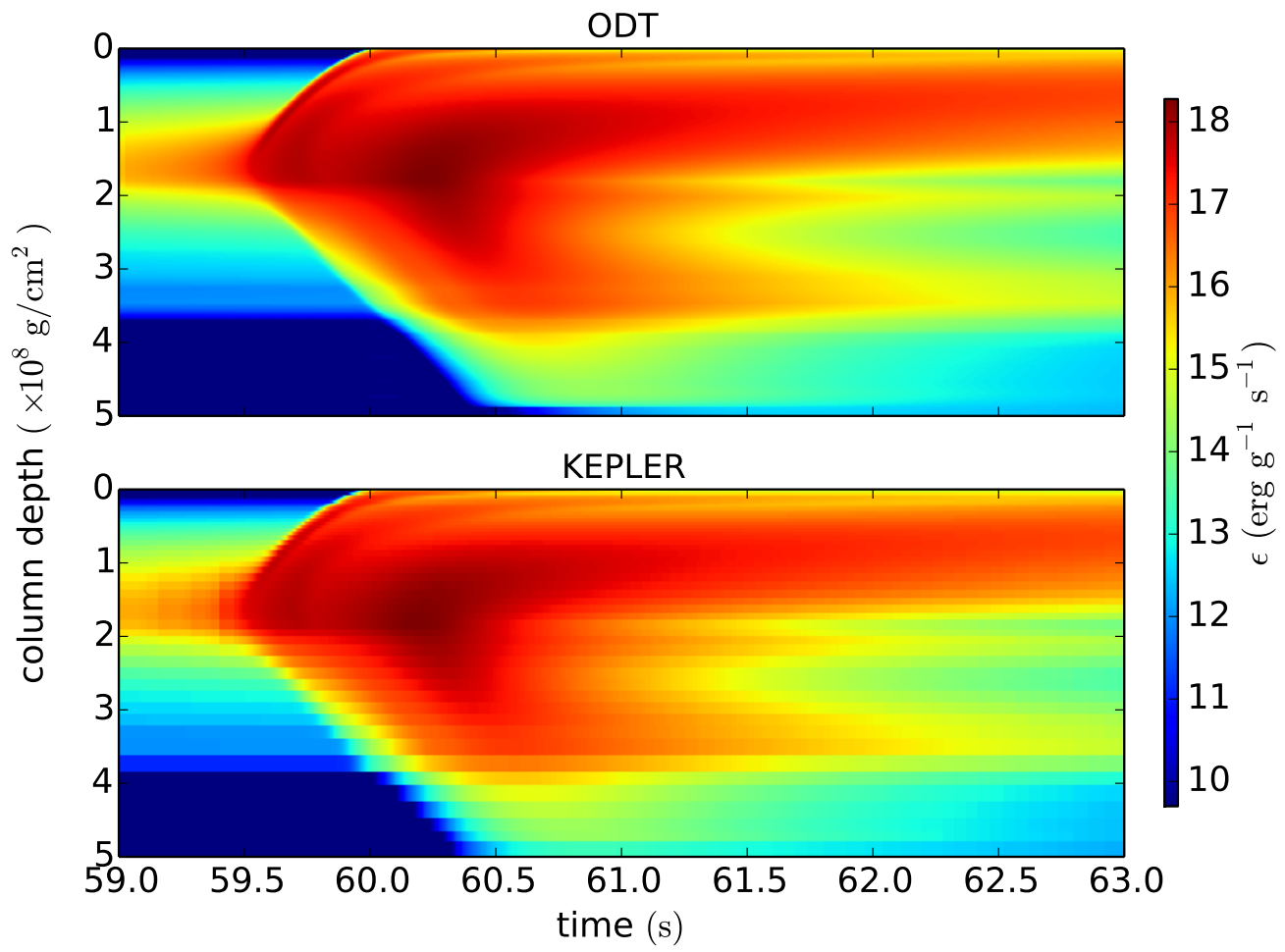

Figure 4.3: Nuclear energy generation rates comparison without turbulent motion. The top panel shows the result of the ODT model, while the bottom panel shows the result of the KEPLER model. The colors represent the energy generation rate $(\epsilon)$ on a logarithmic scale. The horizontal time axis of the ODT model has shifted slightly at $\epsilon=10^{17} \mathrm{erg} \mathrm{g}^{-1} \mathrm{~s}^{-1}$ to match the burning process of the KEPLER model.

because the bottom heating varies at $m=9 \times 10^{8} \mathrm{~g} / \mathrm{cm}^{2}$ in the KEPLER model. Tests show that small variation of heating at this depth doesn't affect the overall burning process in Figure 4.3 because it is dominated by the energy generated from the reactions. The initiation point of the runaway, however, is sensitive to the heating at the bottom. To match the time axes of the burning processes, one of the axes has shifted so that $\epsilon$ reaches $10^{17} \mathrm{erg} \mathrm{g}^{-1} \mathrm{~s}^{-1}$ at the same time. Both bursts initiate at time around $59 \mathrm{~s}$ due to the burning of helium, and reach a peak energy generation rate at time around $61 \mathrm{~s}$ due to the burning of carbon and following isotopes. For a better comparison, Figure 4.4 and Figure 4.5 show the abundance comparisons of the two models at time 


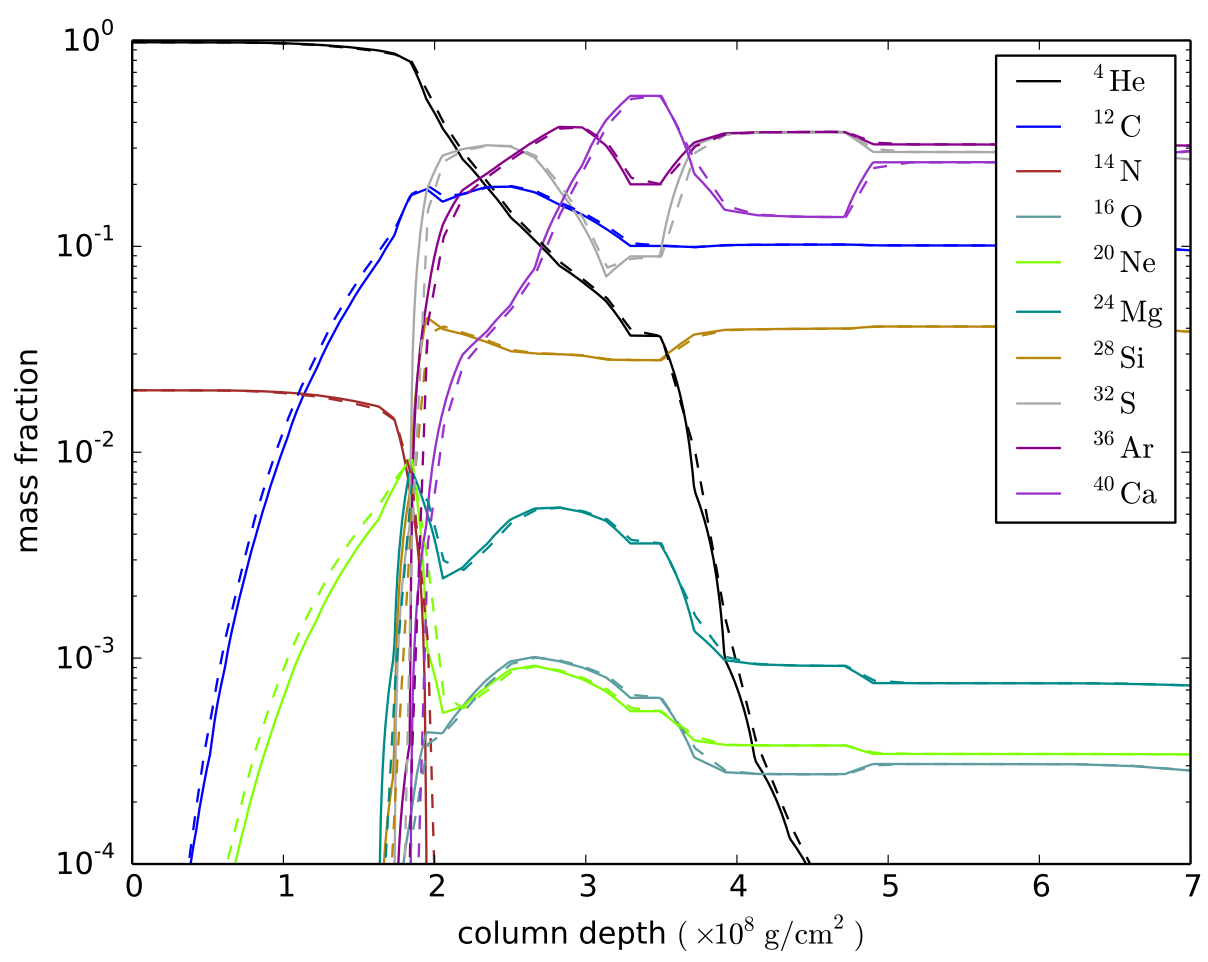

Figure 4.4: Abundance comparison at $55 \mathrm{~s}$ without turbulent motion. The isotopes are selected from the ones with mass fraction above $10^{-4}$. The dashed curves are from the KEPLER model, while the curves are from the ODT model. At $55 \mathrm{~s}$, triple- $\alpha$ reaction dominates and the heat is accumulating for later initiation.

$55 \mathrm{~s}$ and $65 \mathrm{~s}$, respectively. The $\mathrm{x}$-axis is the column depth, and the $\mathrm{y}$-axis is the mass fraction. During these 10 seconds, most of the reactions take place and most of the heat is generated. The dashed curves are from the KEPLER model while the curves are from the ODT model. The isotopes in the figures are selected from the ones that have mass fraction above $10^{-4}$ and are represented by different colors. It is clear from Figure 4.5 that after most of the reactions, the variations of abundance between these two models are still very small.

All the comparisons conclude that these two models produce very similar results despite the obvious resolution difference and the deviations caused by mapping from the initial configuration of the KEPLER model. It is a test not only for the nuclear 


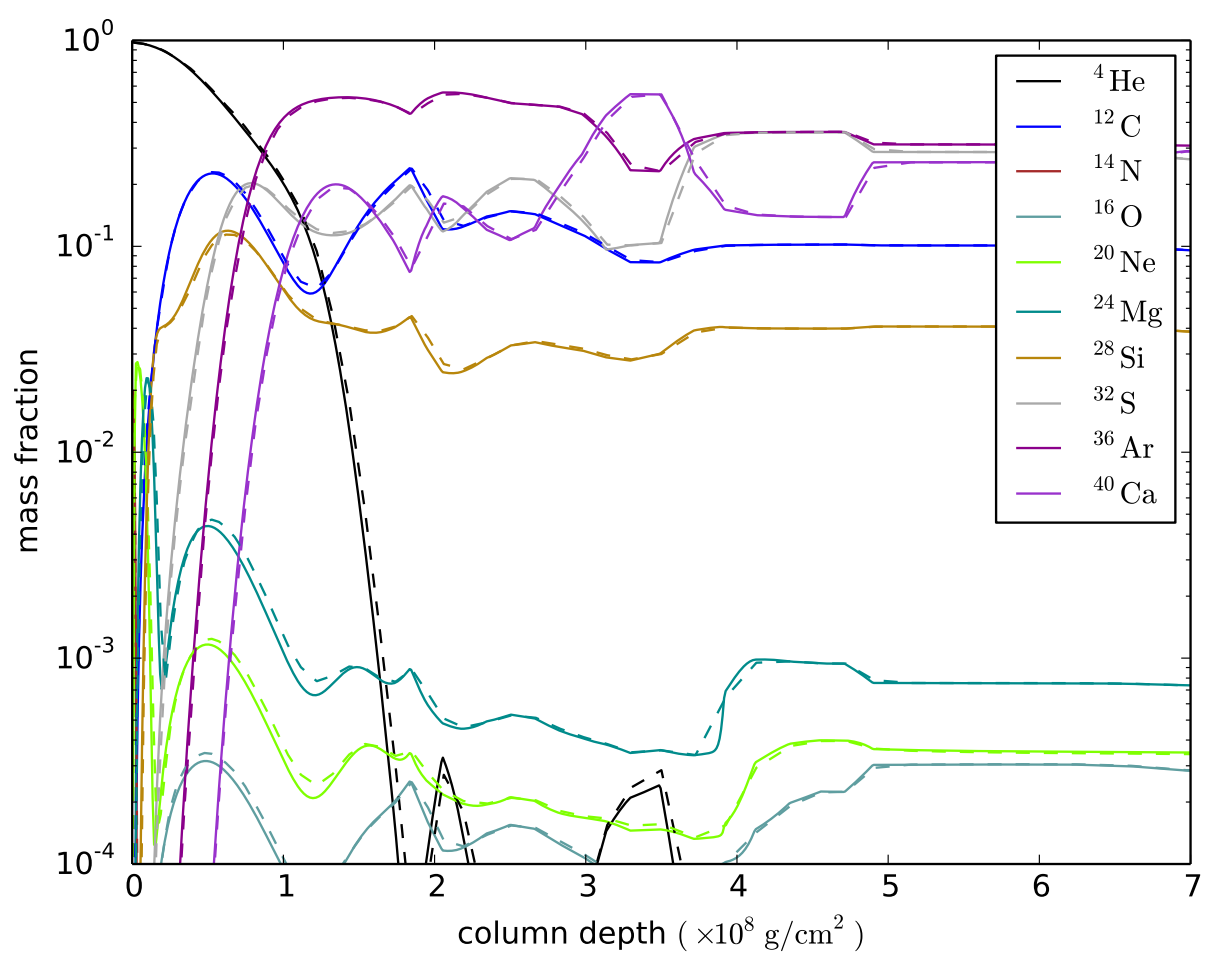

Figure 4.5: Abundance comparison at $65 \mathrm{~s}$ without turbulent motion. The isotopes are selected the same way as in Figure 4.4. At $65 \mathrm{~s}$, most of the reactions in Figure 4.1 have taken place. But the differences in the abundance of all the isotopes are still very small.

networks, but also the diffusion processes that involves the calculation of opacities. Since we have isolated the turbulent motion so far, the discussion of the results in the following sections will mainly focus on the mixing mechanisms.

\subsection{Light curves}

Since there is only one free parameter, $C$ in Equation (4.11), in the ODT models, a survey of this parameter is carried out first. The light curves in the local frame of the star with different $C$ parameters are shown in Figure 4.6. The vertical axes are the luminosities and the horizontal axes are the times since the initial configuration. The top panel shows the light curves from $58 \mathrm{~s}$ to $80 \mathrm{~s}$. The bottom two panels zoom in to 


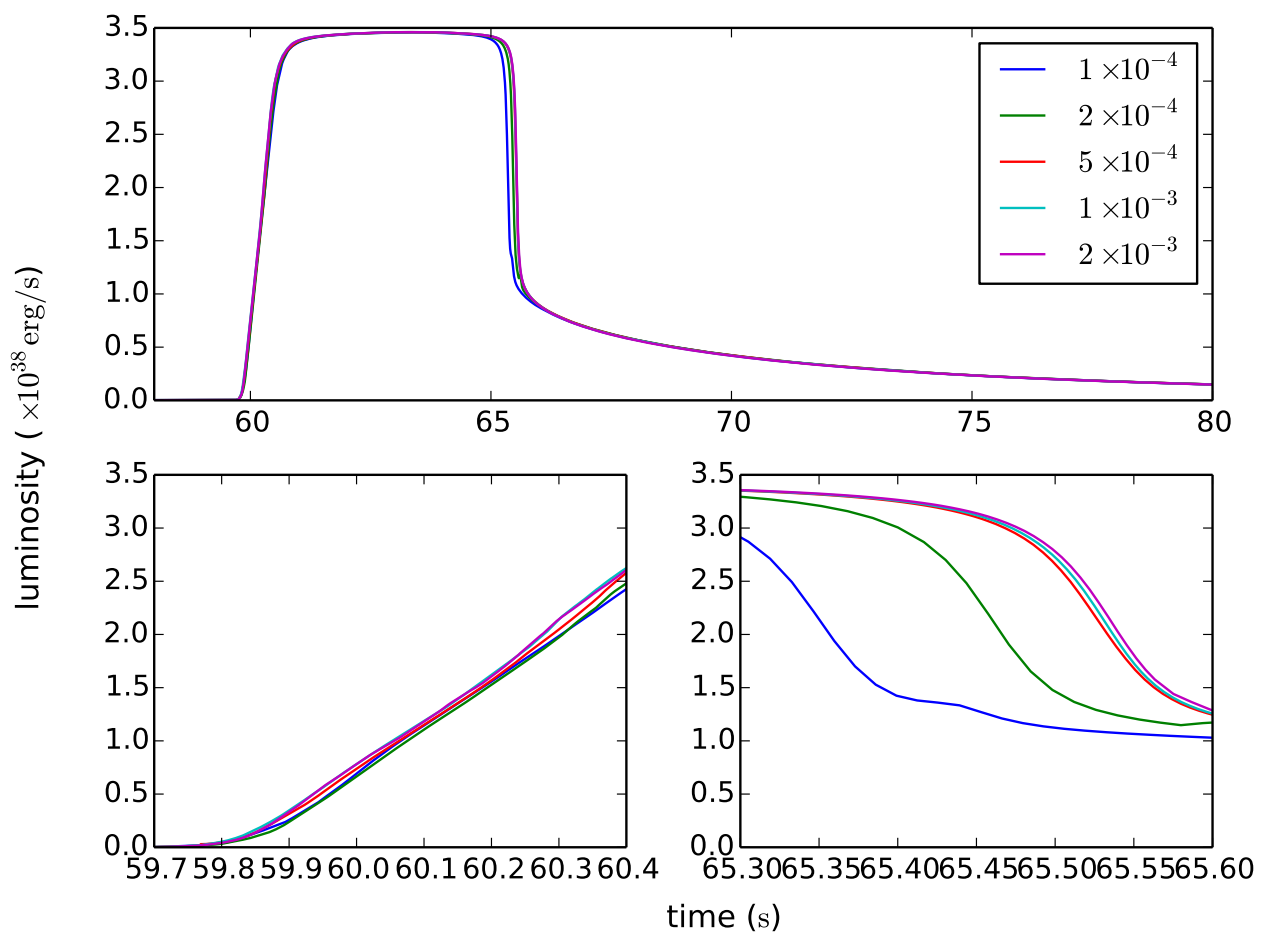

Figure 4.6: Light curves of the ODT models with different $C$ parameters. The top panel shows the overall features of all the light curves. The bottom two panels zoom in to time intervals $[59.7,60.4]$ and $[65.3,65.6]$, respectively, to compare the luminosities in detail. The figure serves as a quick survey of the $C$ parameter and $C=10^{-3}$ is chosen for the simulation of statistical analysis.

time intervals $[59.7,60.4]$ and $[65.3,65.6]$, respectively, to compare the luminosities in detail. Each color curve corresponds to a one-realization simulation with a $C$ parameter. The time resolution for each realization is small due to the small burning time step, so the light curve is smooth even without statistical average. Because the variation of luminosities from the mean values (see Figure 4.7) is not big, it is valid to use one-realization simulation for a quick parameter survey. Overall, the light curves in Figure 4.6 are very similar in that they all have a quick rising (within $1 \mathrm{~s}$ ) in luminosity, a plateau at the peak luminosity (about $3.4 \times 10^{38} \mathrm{erg} / \mathrm{s}$ ) and the duration is about $5 \mathrm{~s}$, and a decay tail converged after $66 \mathrm{~s}$. A careful comparison indicates that both the slope of the rising luminosity around $60 \mathrm{~s}$ and the duration of the plateau increase 


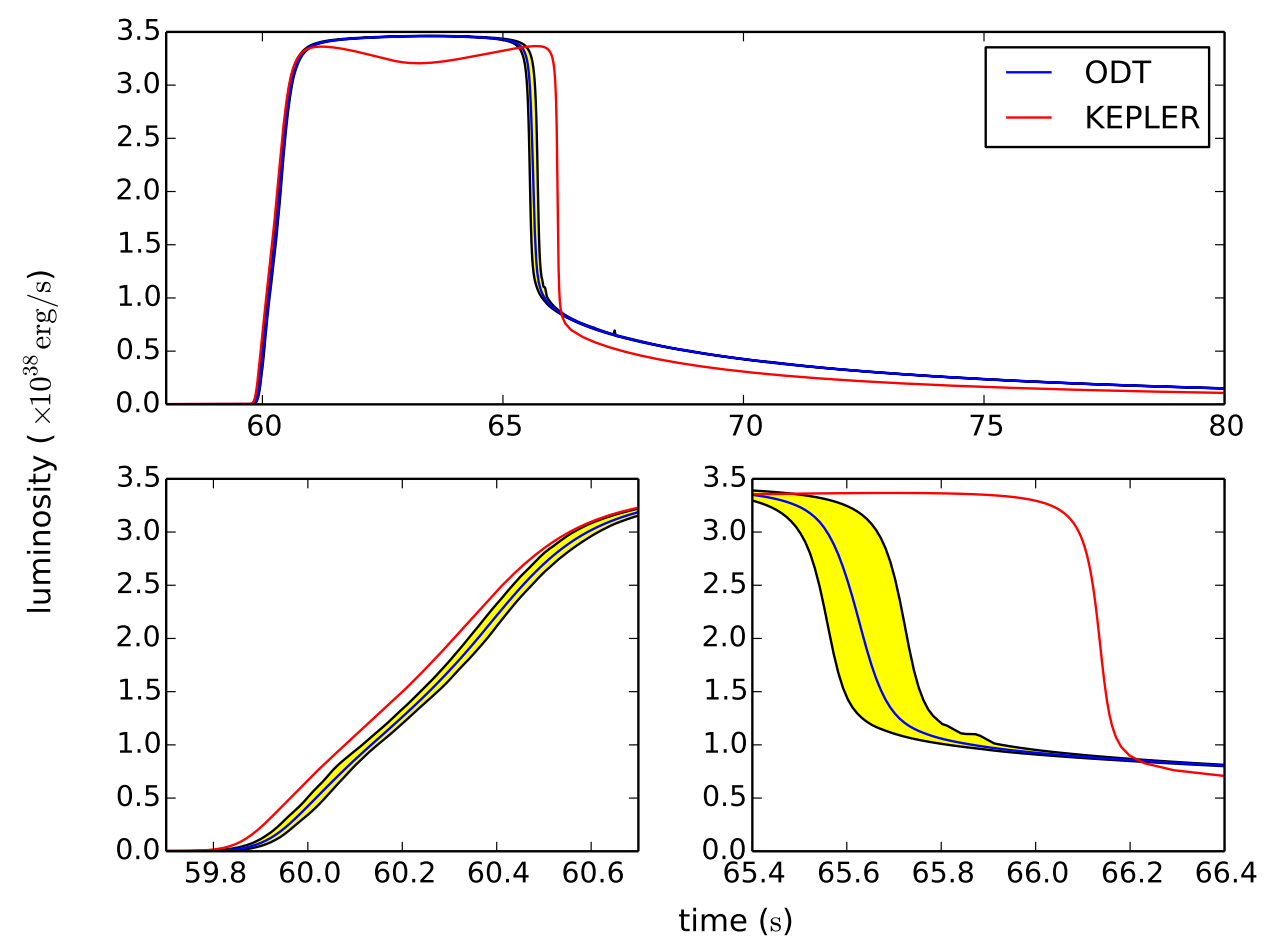

Figure 4.7: Light curves comparison of the ODT model and the KEPLER model. The top panel shows the overall features of the light curves of both models (ODT in blue and KEPLER in red). The light curve of the ODT model is an average of 93 realizations with $C=10^{-3}$. The bottom two panels zoom in to time intervals $[59.7,60.7]$ and $[65.4,66.4]$, respectively, to compare the luminosities in detail. The yellow area around the blue curve covers the variations between all the realizations.

when the $C$ parameter increases. This increase becomes less and less sensitive as $C$ goes above $5 \times 10^{-4}$. Because $C$ controls the overall eddy rate, more eddy events occur and the mean time for eddy sampling decreases as $C$ increases, therefore the computing becomes costly as $C$ gets big. In order to save the computing time and also match the light curve of the KEPLER model (see Figure 4.7), $C=10^{-3}$ is chosen for the following multi-realization simulation. It is worth mentioning that the bigger the $C$ parameter is, the faster the turbulent diffusion becomes. So the choice of $C$ here means that the models discussed in the rest of the chapter have a relatively fast turbulent diffusion. 
In Figure 4.7, the blue curves represent the average light curve of an ODT simulation of 93 realizations. Each realization is specified by a sequence of random numbers generated by the generator with a specific seed (Section 3.2). The shaded yellow area indicates the variations of all the realizations. The red curve is the light curve of the KEPLER model. From the top panel, it is clear that both models have a sharp rising in surface luminosity with a similar time scale. And after reaching peak values $\left(3.3-3.4 \times 10^{38} \mathrm{erg} / \mathrm{s}\right)$, both luminosities stay around their peak values for about $5-6 \mathrm{~s}$, during which the zones near the surface expand and lift up the photosphere above. As the burning energy ceases, the luminosities decay with a longer time scale and the photosphere radius shrinks back to its original size.

Despite the similarities, there are four noticeable differences. First, there is a $\sim 0.1 \mathrm{~s}$ time offset during the rising of the curves (see bottom left panel). Because the time axes are matched according to the time when $\epsilon=10^{17} \mathrm{erg} \mathrm{g}^{-1} \mathrm{~s}^{-1}$ during the burst ignition, this time offset comes from the differences of heat propagation rate towards the surface. During the burst, the heat is mainly driven by turbulent motion of large length scales, so the mixing region in the KEPLER model expands faster at the beginning of the burst. Second, the luminosity reaches a plateau in the ODT model around its peak but the one in the KEPLER model has a dip. When the luminosity reaches the peak value in the ODT model (around $63 \mathrm{~s}$ ), the density of the top zone decreases to $\sim 20 \mathrm{~g} / \mathrm{cm}^{3}$, which corresponds to an expansion equivalent to $\sim 5 \%$ of the star radius. So plane parallel assumption is no longer as valid as before and a deviation from the KEPLER model occurs. Another contribution to this deviation is that the burning near the surface is more energetic in the ODT model owing to the mixing of fuels in this region (see discussion in Section 4.6). Third, the luminosity in the ODT model starts to drop $\sim 0.5 \mathrm{~s}$ earlier than that in the KEPLER model, which leads to a $\sim 0.6 \mathrm{~s}$ difference in the total duration of the peak. The cause is that more fuels are burned in the KEPLER model during the expansion phase (see discussion in Section 4.6). Fourth, the tail of the ODT model following the peak decays more slowly than that of the KEPLER model.

In this chapter, I focus mainly on the comparisons between theoretical models. The comparison between these two models and observation is discussed in the future work of Chapter 5 . 


\subsection{Turbulent motions and abundance}

For a one-realization ODT simulation, the eddy cascade structure can be visualized as a sequence of vertical lines with shorter lines following the longer ones if the time axis is horizontal and the simulation domain is vertical, since each eddy event is instantaneous by assumption. Here I present the statistical average of the previous visualization obtained from 93 realizations in the following method. Divide the turbulent region (from $58 \mathrm{~s}$ to $62 \mathrm{~s}$, from star surface to a column depth $m=2 \times 10^{8} \mathrm{~g} / \mathrm{cm}^{2}$ ) uniformly into $400 \times 200$ bins. Collect the vertical lines from all the realizations and count the number of overlaps between lines and bins. After normalization to unity, the counted numbers are plotted with colors in the top panel of Figure 4.8. The non-turbulent region where radiative transfer dominates is represented by the blank space. The bottom panel shows the mixing regions of the KEPLER model according to different stability criteria and formulation (see Weaver et al. 1978a for details). Both plots show very similar mixing features if only consider full convection (indicated by 'conv') in the KEPLER model. Turbulent regions initiate at $\sim 1.2 \times 10^{8} \mathrm{~g} / \mathrm{cm}^{2}$ slightly after $59 \mathrm{~s}$. After $0.5 \mathrm{~s}$, they expand to zones near the surface and a depth $\sim 1.7 \times 10^{8} \mathrm{~g} / \mathrm{cm}^{2}$. Then the sizes of both convective regions shrink to $\sim 0.5 \times 10^{8} \mathrm{~g} / \mathrm{cm}^{2}$ in column depth within $0.3 \mathrm{~s}$. In the next $0.5 \mathrm{~s}$, another expansion of the convective regions with smaller size takes place. And most of the turbulent motions end around $61 \mathrm{~s}$.

For a better comparison, the following analysis combines the nuclear energy generation rates and abundance, both of which are extracted from a typical one-realization calculation as a representation of the ODT models. Figure 4.9 shows the comparison of the nuclear energy generation rates with the ODT model on the top and the KEPLER model on the bottom. Figures 4.10 to 4.15 are six snapshots of prominent abundance from the surface to a depth at $5 \times 10^{8} \mathrm{~g} / \mathrm{cm}^{2}$ at $59 \mathrm{~s}, 59.8 \mathrm{~s}, 60 \mathrm{~s}, 60.5 \mathrm{~s}, 62 \mathrm{~s}$, and $70 \mathrm{~s}$, respectively. The reason of choosing these times is explained in the following discussion. In all six figures, the abundance of six isotopes is represented by color curves (the one-realization ODT model) and dashed color curves (the KEPLER model). Like turbulent motions, both plots in Figure 4.9 have very similar structures and features. Generally speaking, there are two peaks of energy generation rate $\left(>10^{18} \mathrm{erg} \mathrm{g}^{-1} \mathrm{~s}^{-1}\right)$ around $59.8 \mathrm{~s}$ and $60.5 \mathrm{~s}$, which correspond to Figure 4.11 and Figure 4.13 , respectively. 


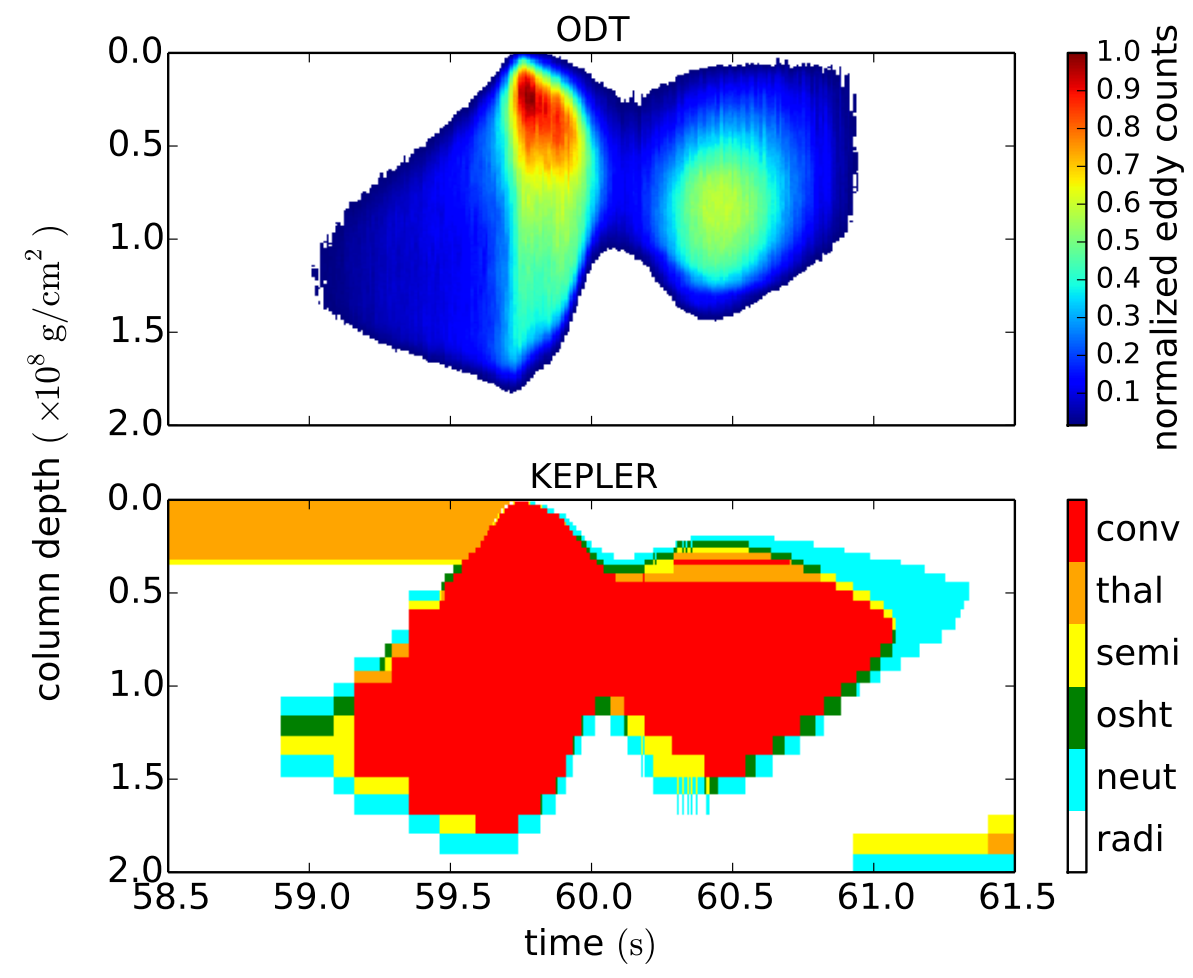

Figure 4.8: Mixing regions comparison. The top panel is the eddy events statistics of ODT models (see text for detailed explanation). The bottom panel is the mixing regions of the KEPLER model according to different stability criteria and formulation. Here 'radi' indicates radiative transfer, 'neut' indicates small amount of convection across radiative zonal interface, 'osht' indicates overshoot mixing, 'semi' indicates semiconvection, 'thal' indicates thermohaline mixing, and 'conv' indicates full convection.

Between the two peaks, there is a valley point at around $60 \mathrm{~s}$ and the corresponding abundance is shown in Figure 4.12.

Starting from $59 \mathrm{~s}$ (see Figure 4.10, helium burns continuously and all the abundance evolves at the same pace in both models. After the convection kicks in at about $59.2 \mathrm{~s}$, the abundance of helium and carbon starts to deviate in the mixing regions (see Figure 4.11). The abundance in the KEPLER model is quickly smeared out for both helium and carbon from $0-1.6 \times 10^{8} \mathrm{~g} / \mathrm{cm}^{2}$, which is consistent with the convective region in Figure 4.8. In the ODT model, the slope of helium abundance in the same region indicates a slower propagation rate of convection towards the surface, which leads to 


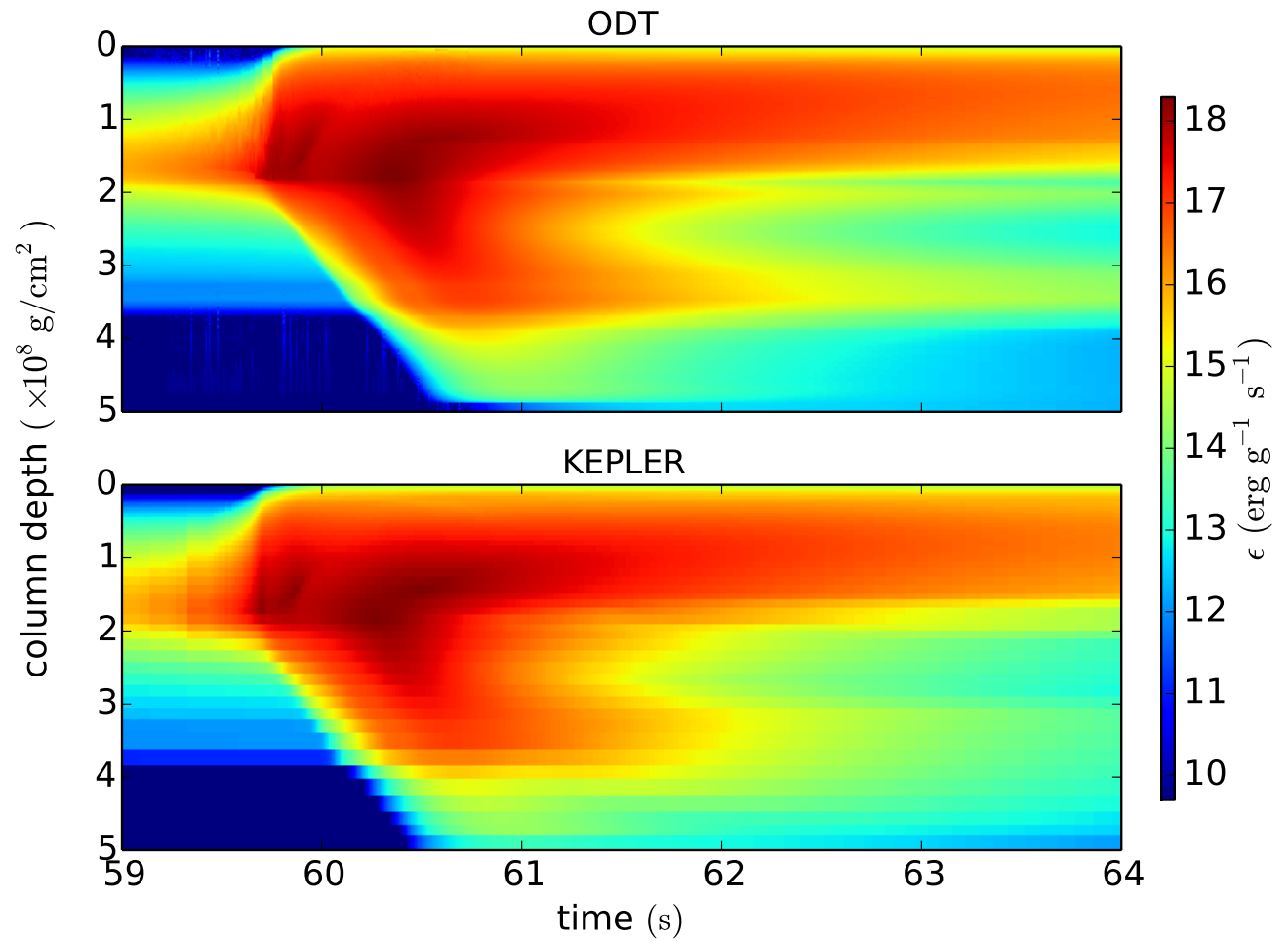

Figure 4.9: Nuclear energy generation rates comparison with turbulent motion. The top panel shows the result from the one-realization ODT model, while the bottom panel shows the result from the KEPLER model. The colors represent the energy generation rate on a logarithmic scale. The horizontal time axis of the ODT model has shifted slightly at $\epsilon=10^{17} \mathrm{erg} \mathrm{g}^{-1} \mathrm{~s}^{-1}$ to match the burning process of the KEPLER model.

a delayed rising of surface luminosity (Figure 4.7). And the differences in helium and carbon abundance indicate that the overall mixing is not as fast as that of the KEPLER model which uses a modified mixing length theory (Weaver et al. 1978a). In other words, the turbulent diffusivity is smaller in the ODT models during this process. All the abundance below $2 \times 10^{8} \mathrm{~g} / \mathrm{cm}^{2}$ stays the same because of the small microscopic diffusivities. Once the ignition triggered, not only the surface zones, but also the density around the burning center keeps decreasing at a high rate due to expansion. Meanwhile, the temperature keeps increasing at a high rate due to the heat generated. After a certain point $\left(T \sim 10^{9} \mathrm{~K}\right)$, the EOS starts to reduce the increase rate of temperature so 


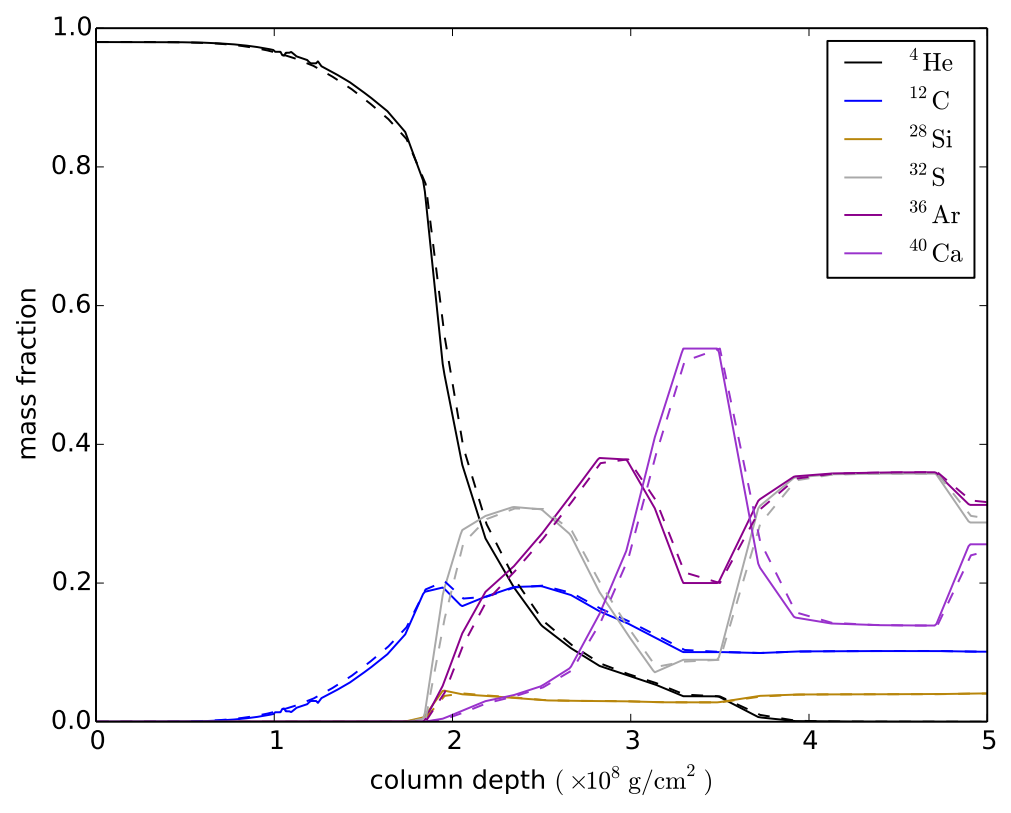

Figure 4.10: Prominent abundance at 59s (before the first peak). The color curves correspond to the ODT model while the dashed color curves correspond to the KEPLER model.

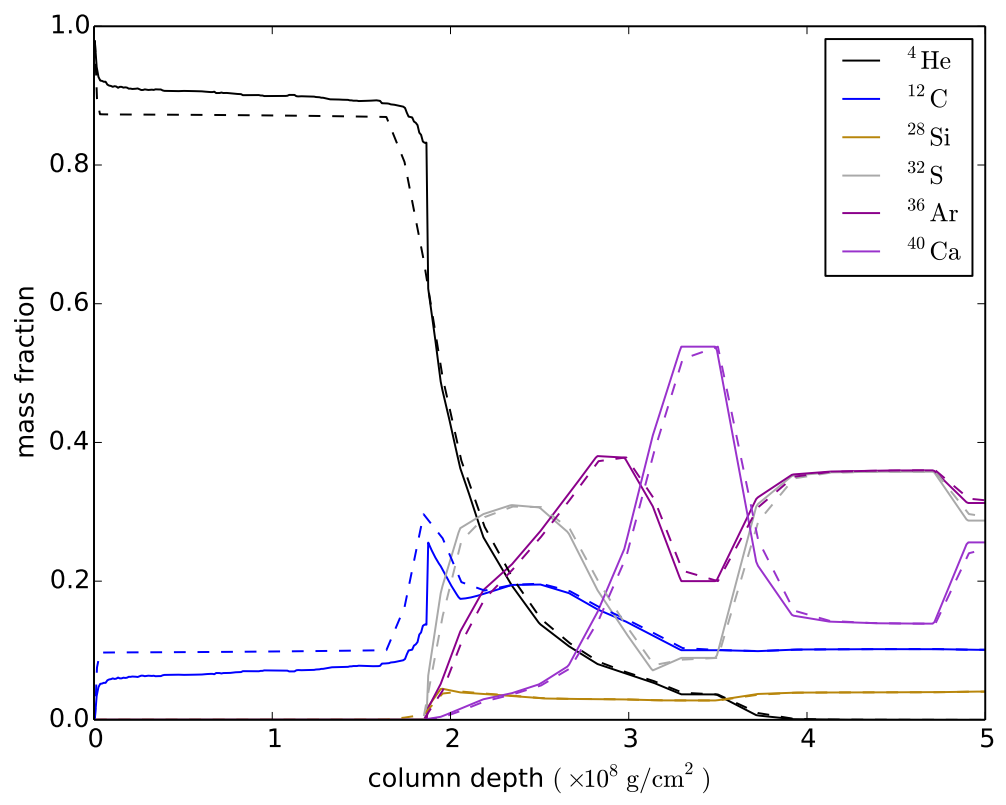

Figure 4.11: Prominent abundance at 59.8s (around the first peak). The color curves correspond to the ODT model while the dashed color curves correspond to the KEPLER model. 


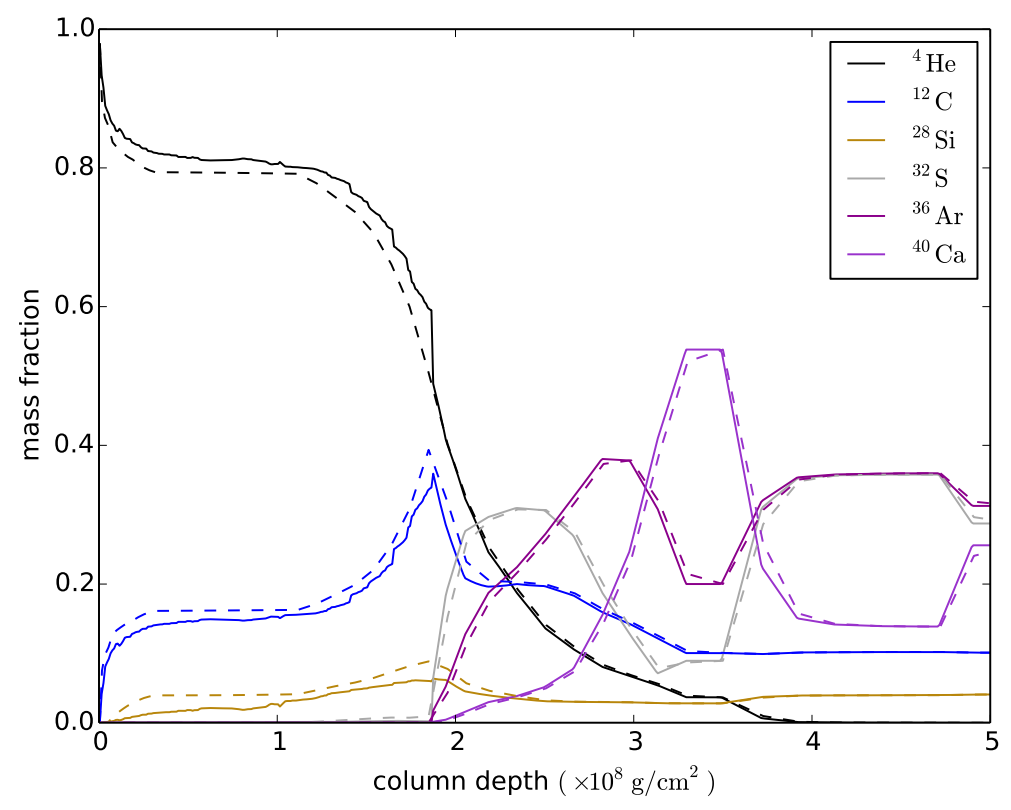

Figure 4.12: Prominent abundance at $60 \mathrm{~s}$ (around the valley). The color curves correspond to the ODT model while the dashed color curves correspond to the KEPLER model.

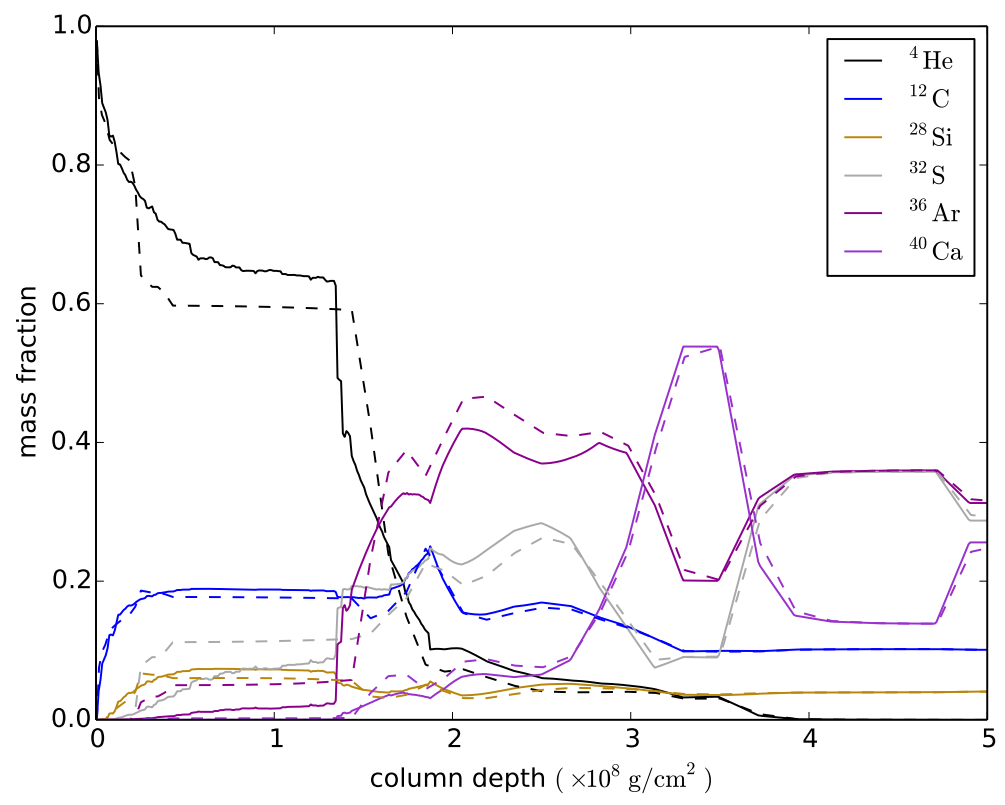

Figure 4.13: Prominent abundance at $60.5 \mathrm{~s}$ (around the second peak). The color curves correspond to the ODT model while the dashed color curves correspond to the KEPLER model. 


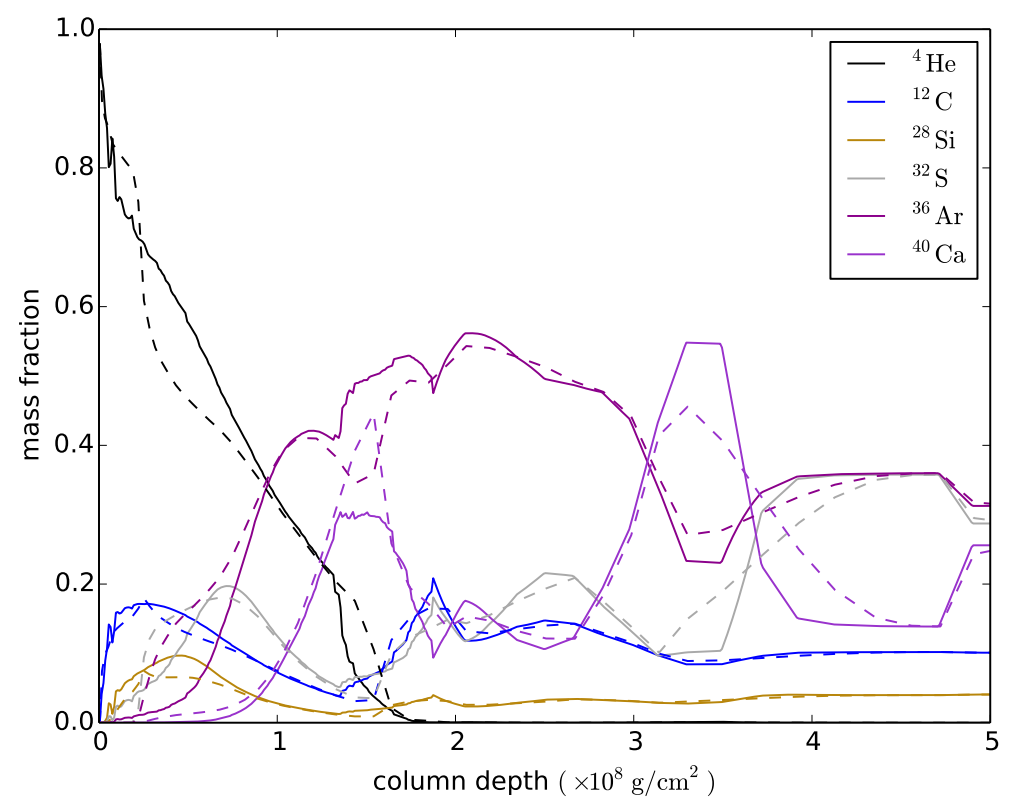

Figure 4.14: Prominent abundance at $62 \mathrm{~s}$ (after the second peak). The color curves correspond to the ODT model while the dashed color curves correspond to the KEPLER model.

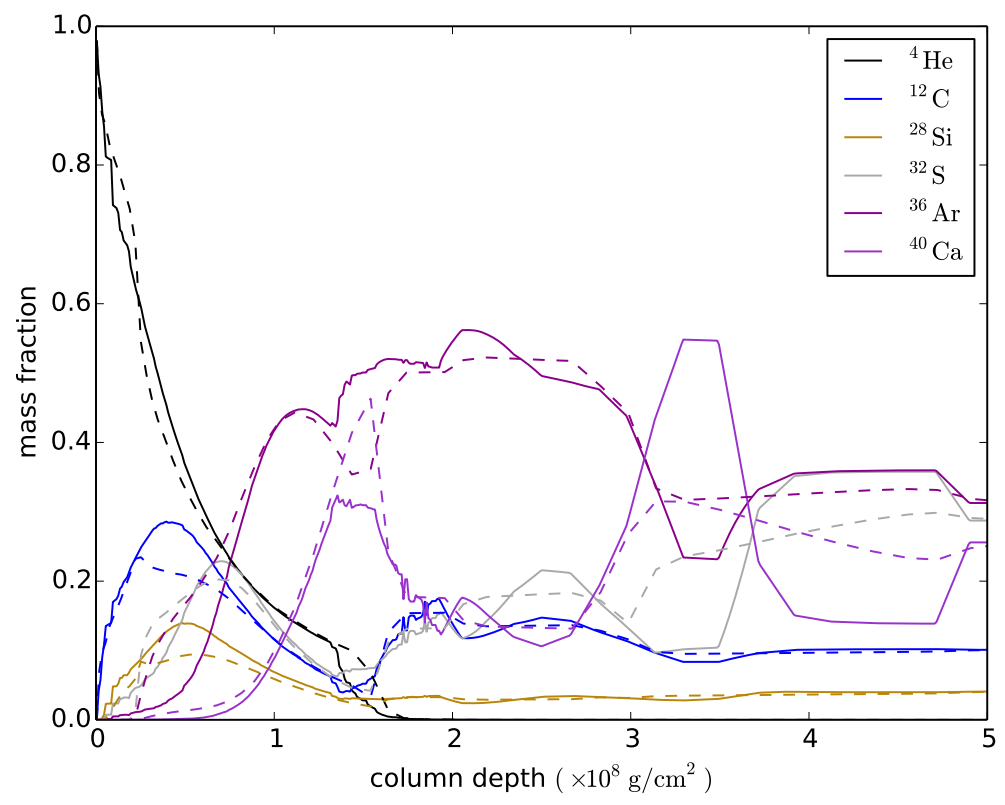

Figure 4.15: Prominent abundance at $70 \mathrm{~s}$. The color curves correspond to the ODT model while the dashed color curves correspond to the KEPLER model. 
much that the triple- $\alpha$ reaction rate starts to decrease from the peak value due to the decrease of density. At $60 \mathrm{~s}, \epsilon$ reaches the valley even though the temperature is still increasing. It is worth mentioning that the convective region in Figure 4.8 also shrinks to a local minimum owing to the coupling between mixing and burning. Since most of the mixing occurs at locations where the entropy gradient with respect to radius is decreasing, the two big turbulent regions correspond to the two 'hot spots' of nuclear burning. After the first 'hot spot', carbon starts to accumulate especially right below the mixing region (around $1.8 \times 10^{8} \mathrm{~g} / \mathrm{cm}^{2}$ in Figure 4.12 where temperature is high to burn helium fast and the microscopic diffusion of abundance is small. As the temperature keeps rising above $10^{9} \mathrm{~K}$, the reaction ${ }^{12} \mathrm{C}(\alpha, \gamma){ }^{16} \mathrm{O}$ becomes more and more violent and its reaction rate contributes dominantly to the overall energy generation rate. Therefore $\epsilon$ increases again and the burning goes into the second 'hot spot'. At $60.5 \mathrm{~s}$ when the temperature reaches its peak $\left(\sim 1.5 \times 10^{9} \mathrm{~K}\right)$, the convective region indicated by the plateau (see Figure 4.13) in the KEPLER model spans only from 0.5$1.5 \times 10^{8} \mathrm{~g} / \mathrm{cm}^{2}$. But the eddy events in the ODT model can reach $\sim 0.1 \times 10^{8} \mathrm{~g} / \mathrm{cm}^{2}$ and bring down more helium from the top layers, which increases $\epsilon$ in the region near the surface $\left(0.1-0.2 \times 10^{8} \mathrm{~g} / \mathrm{cm}^{2}\right)$. More burning near the surface yields higher surface luminosity, which also contributes to the deviation of light curves at the peak luminosity (see Figure 4.7). In the convective region, the abundance diffusion indicates again that the turbulent diffusivity is higher in the KEPLER model than that in the ODT model. About $20 \%$ of helium is burned, but the mass fraction goes mostly to sulfur because carbon cannot survive much at this temperature. After the temperature reaches $10^{9} \mathrm{~K}$, the time offset developed from the first 'hot spot' starts to influence the abundance of heavier elements in the non-turbulent region below as the abundance changes once the heat wave raises the zone temperature above a certain threshold. All turbulent mixing ceases after $61 \mathrm{~s}$ because of the depletion of helium layer. But the nuclear burning is still ongoing because of the high temperature. Figures 4.14 and 4.15 show the stable burning in the following $1 \mathrm{~s}$ and $9 \mathrm{~s}$, respectively. Here I focus on the region from the surface to $10^{8} \mathrm{~g} / \mathrm{cm}^{2}$ where the abundance deviates mostly due to mixing differences. In the region from $0.2-1 \times 10^{8} \mathrm{~g} / \mathrm{cm}^{2}$, more helium is left in the ODT model at $62 \mathrm{~s}$. As the temperature keeps decreasing during the cooling phase, more carbon can survive at this depth. In deeper layers where temperature is still high, carbon burns quickly 
into heavier isotopes, so the differences are shown in the abundance of sulfur, argon, and calcium. At $70 \mathrm{~s}$ (Figure 4.15 when the expansion is over, about $10 \%$ more carbon is produced at $0.5 \times 10^{8} \mathrm{~g} / \mathrm{cm}^{2}$ in the ODT model. This number will slightly increase during the rest of cooling phase. The survival of more carbon during the expansion indicates that more fuels are burned in the KEPLER model, so the total expansion duration is slightly longer, which is consistent with the longer peak duration in the light curves comparison (Figure 4.7).

The importance of more carbon produced in XRBs is that carbon is considered the fuels of the more energetic burst phenomena called superbursts Cumming \& Bildsten 2001). Superbursts were first observed in 2000 (Cornelisse et al. 2000). Comparing with regular bursts, they usually have total energy $\sim 1000$ times more and last $\sim 1000$ times longer. Their ignition depths are of order $10^{12} \mathrm{~g} / \mathrm{cm}^{2}$ where hydrogen and helium are unable to survive. Carbon accumulation in the deep layers can either comes from accretion, or from the products of regular bursts. Here I only discuss the latter. Some previous KEPLER models by Alexander Heger and Laurens Keek show that the bursts did not make enough carbon to ignite superbursts. So more carbon produced from the ODT model can possibly make the accumulated carbon approach the ignition threshold if the extra amount of carbon survives the descending.

\subsection{Conclusion}

In this chapter, I presented the first XRB simulation using ODT as the turbulent mechanism. The models are built on the basis of a KEPLER model for pure helium bursts with photospheric radius expansion. I compared the nuclear networks, light curves, turbulent motions, and abundance in detail. The ODT model successfully produced a photospheric radius expansion burst triggered by the $\alpha$-chain reactions. The peak luminosity also well matched the Eddington luminosity $\left(\sim 3.5 \times 10^{38} \mathrm{erg} / \mathrm{s}\right)$ without hydrogen in the atmosphere. Comparing with the KEPLER model, the ODT model showed good agreement on the luminosity rising and decay time scales, as well as total burst duration. Particularly, I focused on the turbulent motions comparison between the ODT mechanism and the mixing length theory used in KEPLER, and relate it to the differences found in the light curves and abundance. The mixing in the ODT model 
shows a comparatively slower turbulent diffusion in terms of the propagation rate of convection towards the surface and the turbulent diffusivities within the mixing region. Besides, mixing in ODT can expand further to the surface of the star where convection is prohibited by the stability criteria in KEPLER. These differences lead to the result that more carbon is produced on the top layers of the star during the burst. As carbon descends and accumulates to a certain threshold in the deeper layers, it triggers the unstable burning that results in a much more energetic superburst. 


\section{Chapter 5}

\section{Summary and Future Work}

The objective of this dissertation is to present simulations of the photospheric radius expansion bursts with a new hydrodynamic model, the one-dimensional turbulence (ODT) model, which is applied to Type I X-ray bursts (XRBs) simulations also for the first time. Since I almost started from scratch, a lot of the work was focused on the development of this model. For a new model to be accepted, validation is of great importance and necessity. So tests have been performed carefully after each step of the development process. As discussed in Chapter 3, I started with building a basic ODT model on a Lagrangian grid (Section 3.1). Then I tested the new configuration with the simulations of the Rayleigh-Taylor instabilities (Section 3.3). The results showed that the ODT model produced similar propagation rates of the mixing thickness as those of 3D direct numerical simulations. Next, I implemented the Helmholtz equation of state (Section 3.4) for stellar environment and an implicit solver for diffusion processes (Section 3.5). The former replaced the ideal gas equation of state and the latter replaced an explicit diffusion solver to get rid of the limit of the Courant-Friedrichs-Lewy time. After tests of these two pieces separately, a simulation of an XRB that involved three-isotope burning was performed to test the compatibility of them to the basic ODT model. Furthermore, I included a nuclear network with 19 isotopes and found that the burning process was almost identical as that in the KEPLER model (Section 4.1). Finally, I was confident to simulate a pure helium burst with the ODT model and compared the results with the KEPLER model (Chapter 4). Both models showed good agreement on the light curves and turbulent regions. More importantly, the mixing mechanism in the 
ODT model has a slower mixing process but the mixing expands further to the surface region of the star, which causes more carbon produced after one burst. This result is important in that more carbon will accumulate if it descends to deeper layers of the star and it will become the fuels of superbursts.

There is still lots of work needed to be done in the future to improve the ODT model. Because some of the discussion below includes results from KEPLER runs only, I put them after the summary to maintain the focus of the dissertation on ODT.

In Chapter 4, the ODT model shows that more carbon is produced near the surface of the star after one burst. Unfortunately this argument alone cannot guarantee that the carbon abundance will increase in the deep layers. The question now is that how much of these ashes can survive when they reach the depth of order $10^{12} \mathrm{~g} / \mathrm{cm}^{2}$, since the actual amount of carbon abundance at this depth determines the ignition of superbursts. The original KEPLER model is built to study the ultracompact binary $4 \mathrm{U} 1820-30$ which is famous for its 176 day X-ray intensity modulation (Priedhorsky \& Terrell 1984). And regular XRBs are observed only in its low flux state. Currently, there is no modulation obtained in the KEPLER model, so I consider low flux state only. It is assumed that no carbon survives during the XRBs phase because of the high temperatures, whereas a certain amount of carbon is produced in the stable burning phase when no XRB takes place (Strohmayer \& Brown 2002). This assumption is based on the calculation of helium burning evolution in an accreting neutron star with a similar nuclear network (Joss 1978). The only differences are that two nuclear reactions, ${ }^{12} \mathrm{C}+{ }^{12} \mathrm{C}$ and ${ }^{12} \mathrm{C}+{ }^{16} \mathrm{O}$, are not included in the 19-isotope network (Section 4.1). But their contribution to carbon consumption is negligible because of small nuclear reaction rates. After running the KEPLER model for $\sim 3000$ bursts, the descending carbon reaches $\sim 5.5 \times 10^{11} \mathrm{~g} / \mathrm{cm}^{2}$ with a mass fraction $\sim 12 \%$, which indicates that there is still fair amount of carbon survives the unstable burning phase in the model. Besides, the argument of no carbon product in the XRBs phase relies on the high temperatures during the bursts which the extra carbon produced in the ODT model survives. Thus more carbon should possibly accumulate in the deeper layers with the ODT mixing mechanism. It is, however, still tempting to simulate the descending process in the ODT model until the ashes reach the ignition depth.

Another improvement is near the surface of the star. I only performed comparisons of 


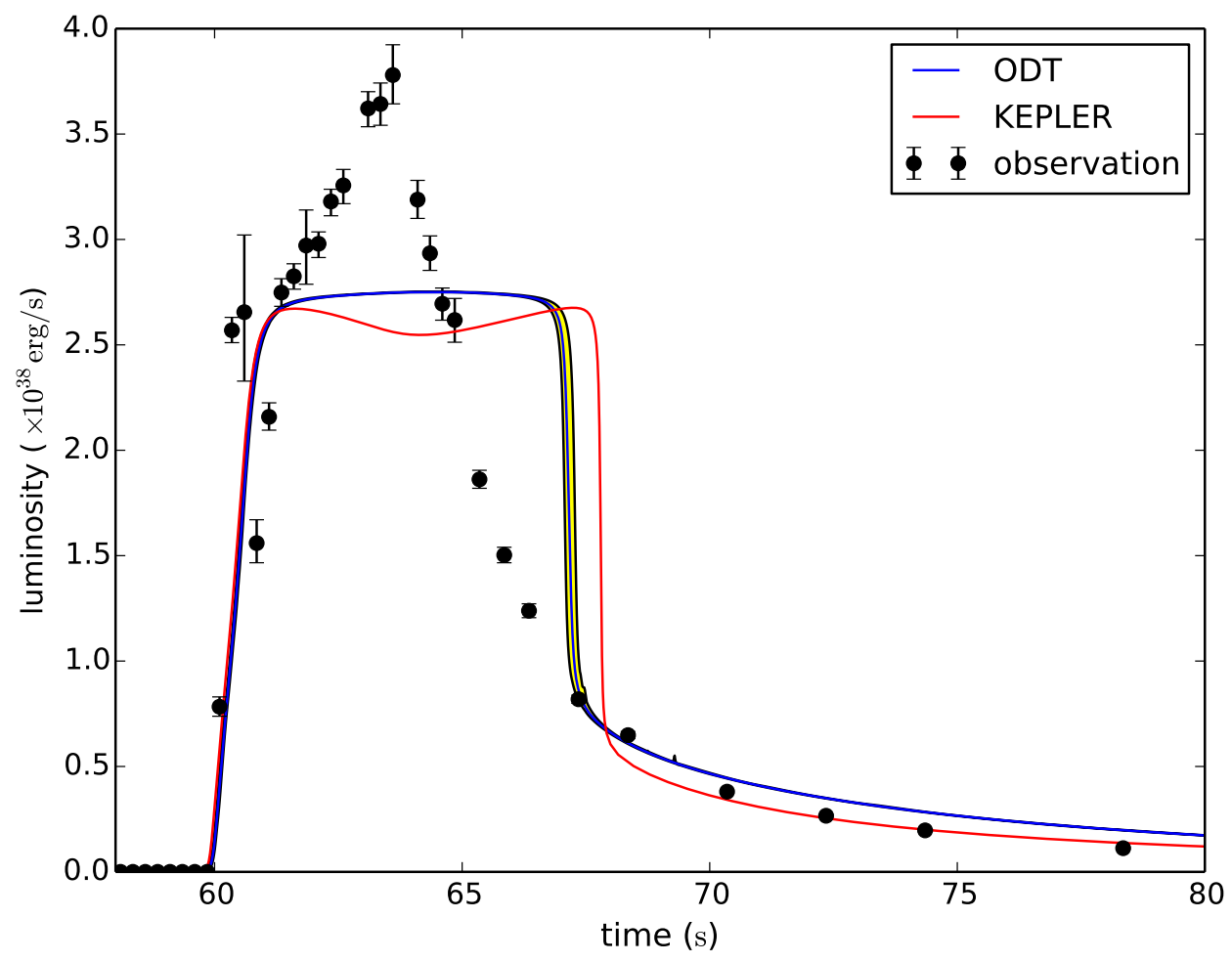

Figure 5.1: light curves comparison with observations. The light curves of the ODT model and the KEPLER model are represented by the blue and red curves, respectively. The observational data are represented by the black dots with error bars. General relativistic correction has been applied to both models.

the light curves between two theoretical models in Chapter 4. Here I compare them with observations in Figure 5.1. The observational data are provided by Duncan Galloway. Luminosities are calculated by

$$
L=4 \pi d^{2} F
$$

where $d$ is the distance from the source to the observer, $F$ is the flux received by the observer. For a better match of peak luminosities, $d$ is chosen as $7 \mathrm{kpc}$ which is within the region of $7.4 \pm 0.4 \mathrm{kpc}$ (Kuulkers et al. 2003). Because the gravity used in both models is Newtonian, the time is dilated by a redshift factor of $(1+z)$, and the luminosities are reduced also by $(1+z)$ when general relativistic correction is considered (Keek \& Heger 
2011). Here $z=0.25835$ for a neutron star of $1.4 M_{\odot}$ and $10^{6} \mathrm{~cm}$ radius. Similar to Figure 4.7, the light curves of the ODT model and the KEPLER model are represented by the blue and red curves, respectively. The observational data are represented by the black dots with error bars. Although a small $d$ is chosen, the peak luminosities are still higher in observation. The overall energies are approximately the same considering the area below. There is an obvious deviation during the expansion phase between the models and observations. Currently, I notice that the peak luminosities during the expansions are sensitive to the choice of the surface resolution for both models. Furthermore, tests with KEPLER models show that the light curves can have a much better fit during the expansion if the resolution of surface zones is reduced by over 1000, which also indicates that the expansion is stronger in the observation and it is consistent with the apparent black-body radius (Kuulkers et al. 2003). So the best way to improve the light curve of the ODT model is to resolve the surface area with finer zones.

To make the two points above possible, a non-uniform grid must be implemented in ODT models. The current ODT model with a uniform Lagrangian grid is convenient to use because it simplifies triplet maps into permutations of grid points. But it limits the resolution as well as simulation domain. In Chapter 4, the resolution in the ODT model is fixed by the top zone $\left(\sim 10^{6} \mathrm{~g} / \mathrm{cm}^{2}\right)$ of the KEPLER model, which means that 900 zones are required to cover a simulation domain of $9 \times 10^{8} \mathrm{~g} / \mathrm{cm}^{2}$. It leads to more computing time even the domain corresponds only to the top layers of the KEPLER model. So a non-uniform grid can greatly reduce the computing time. If the simulation domain can expand to deeper layers of the star (reaching the crust), the crust heating which is an important parameter of XRBs can be directly used as the bottom boundary condition. The difficulty of this implementation lies in that an adaptive mesh should also be included to make a non-uniform grid work. Current attempts in the application of a non-uniform grid alone result in some instabilities in the EOS solver, especially with $(P, h)$ pair as input (Section 3.4) which requires a good initial guess for fast convergence. Because it is easy to cause a sudden big change in the profiles if an eddy event with different zone sizes occurs in a region where density gradient is big.

Finally, it is also required to simulate multiple bursts for studying the descending process of ashes. The current ODT model can only resolve one burst event and the initial configuration is based on a KEPLER model. The important parameter, accretion rate, 
is not playing an explicit role in the simulation. Besides, the interactions between bursts also influence the abundance of ashes as they descend. It is possible to involve accretion into the scheme by modifications of the abundance diffusion with a different boundary condition on the top. 


\section{References}

Alastuey, A., \& Jancovici, B. 1978, apj, 226, 1034

Andrews, M. J., \& Spalding, D. B. 1990, Physics of Fluids A, 2, 922

Arnett, D., Meakin, C., \& Young, P. A. 2009, apj, 690, 1715

Ashurst, W. T., \& Kerstein, A. R. 2005, Physics of Fluids, 17, doi:http://dx.doi.org/10.1063/1.1847413

Belian, R. D., Conner, J. P., \& Evans, W. D. 1976, apjl, 206, L135

Böhm-Vitense, E. 1958, zap, 46, 108

Buchmann, L. 1996, apjl, 468, L127

Calder, A. C., Fryxell, B., Plewa, T., et al. 2002, The Astrophysical Journal Supplement Series, 143, 201

Caughlan, G. R., \& Fowler, W. A. 1988, Atomic Data and Nuclear Data Tables, 40, 283

Chandrasekhar, S. 1955, Mathematical Proceedings of the Cambridge Philosophical Society, 51, 162

Chenevez, J., Altamirano, D., Galloway, D. K., et al. 2011, mnras, 410, 179

Christy, R. F. 1966, apj, 144, 108

Clark, G. W., Jernigan, J. G., Bradt, H., et al. 1976, apjl, 207, L105

Clement, M. J. 1993, apj, 406, 651 
Cook, A. W., Cabot, W., \& Miller, P. L. 2004, Journal of Fluid Mechanics, 511, 333

Cook, A. W., \& Dimotakis, P. E. 2001, J. FLUID MECH, 443, 99

Cook, A. W., \& Youngs, D. 2009, Scholarpedia, 4, 6092

Cornelisse, R., Heise, J., Kuulkers, E., Verbunt, F., \& in't Zand, J. J. M. 2000, aap, $357, \mathrm{~L} 21$

Cornelisse, R., in’t Zand, J. J. M., Verbunt, F., et al. 2003, aap, 405, 1033

Courant, R., Friedrichs, K., \& Lewy, H. 1928, Mathematische Annalen, 100, 32

Cumming, A., \& Bildsten, L. 2001, The Astrophysical Journal Letters, 559, L127

Curl, R. 1963, AlChE J., 9, 175

Dalziel, S. B. 1993, Dynamics of Atmospheres and Oceans, 20, 127

Dimonte, G., Youngs, D. L., Dimits, A., et al. 2004, Physics of Fluids, 16, 1668

Duff, I. S., Erisman, A. M., \& Reid, J. K. 1986, Direct Methods for Sparse Matrices (New York, NY, USA: Oxford University Press, Inc.)

Ebisuzaki, T., \& Nakamura, N. 1988, apj, 328, 251

Fryxell, B., Olson, K., Ricker, P., et al. 2000, apjs, 131, 273

Galloway, D. K., Muno, M. P., Hartman, J. M., Psaltis, D., \& Chakrabarty, D. 2008, apjs, 179,360

Glimm, J., Grove, J. W., Li, X. L., Oh, W., \& Sharps, D. H. 2001, J. Comput. Phys., 169,652

Gonzalez-Juez, E., Kerstein, A., \& Lignell, D. 2013, Geophysical \& Astrophysical Fluid Dynamics, 107, 506

Gonzalez-Juez, E., Kerstein, A. R., \& Lignell, D. O. 2011, Journal of Fluid Mechanics, 677,218

Graboske, H. C., Dewitt, H. E., Grossman, A. S., \& Cooper, M. S. 1973, apj, 181, 457 
Grindlay, J., \& Heise, J. 1975, iaucirc, 2879, 1

Gursky, H., Schnopper, H., \& Parsignault, D. 1975, apjl, 201, L127

Hansen, C. J., \& van Horn, H. M. 1975, apj, 195, 735

Heger, A., Cumming, A., Galloway, D. K., \& Woosley, S. E. 2007, apjl, 671, L141

Heger, A., Langer, N., \& Woosley, S. E. 2000, apj, 528, 368

Hoffman, J. A., Lewin, W. H. G., \& Doty, J. 1977, apjl, 217, L23

Hoffman, J. A., Marshall, H. L., \& Lewin, W. H. G. 1978, nat, 271, 630

Holmes, J. A., Woosley, S. E., Fowler, W. A., \& Zimmerman, B. A. 1976, Atomic Data and Nuclear Data Tables, 18, 305

Iben, Jr., I. 1975, apj, 196, 525

in 't Zand, J. 2011, ArXiv e-prints, arXiv:1102.3345

Itoh, N., Totsuji, H., Ichimaru, S., \& Dewitt, H. E. 1979, apj, 234, 1079

James, F. 1990, Computer Physics Communications, 60, 329

Joss, P. C. 1978, apjl, 225, L123

Joss, P. C., \& Melia, F. 1987, apj, 312, 700

Keek, L., \& Heger, A. 2011, apj, 743, 189

Kelton, W. D., \& Law, A. M. 2000, Simulation modeling and analysis (McGraw Hill Boston)

Kerstein, A., \& Wunsch, S. 2006, Boundary-Layer Meteorology, 118, 325

Kerstein, A. R. 1991, Journal of Fluid Mechanics, 231, 361

—. 1999, Journal of Fluid Mechanics, 392, 277

Kerstein, A. R., Ashurst, W. T., Wunsch, S., \& Nilsen, V. 2001, Journal of Fluid Mechanics, 447, 85 
Kerstein, A. R., \& Dreeben, T. D. 2000, Physics of Fluids, 12, 418

Knuth, D. E. 1997, The Art of Computer Programming, Volume 2 (3rd Ed.): Seminumerical Algorithms (Boston, MA, USA: Addison-Wesley Longman Publishing Co., Inc.)

Kuulkers, E., den Hartog, P. R., in't Zand, J. J. M., et al. 2003, aap, 399, 663

Lattimer, J. M., \& Prakash, M. 2001, apj, 550, 426

LeVeque, R., Steiner, O., Gautschy, A., \& für Astrophysik und Astronomie, S. G. 1998, Computational Methods for Astrophysical Fluid Flow: Saas-Fee Advanced Course 27. Lecture Notes 1997 Swiss Society for Astrophysics and Astronomy, Lecture notes / Saas-Fee Advanced Course (Springer)

Levermore, C. D., \& Pomraning, G. C. 1981, apj, 248, 321

Lewin, W. H. G. 1976, iaucirc, 2918, 2

Lewin, W. H. G. 1977, in Annals of the New York Academy of Sciences, Vol. 302, Eighth Texas Symposium on Relativistic Astrophysics, ed. M. D. Papagiannis, 210-227

Lewin, W. H. G., Clark, G., \& Doty, J. 1976, iaucirc, 2922, 1

Lewin, W. H. G., \& Joss, P. C. 1981, ssr, 28, 3

Lewin, W. H. G., Vacca, W. D., \& Basinska, E. M. 1984, apjl, 277, L57

Lewin, W. H. G., van Paradijs, J., \& Taam, R. E. 1993, ssr, 62, 223

Lin, D. J., Bayliss, A., \& Taam, R. E. 2006, apj, 653, 545

Malone, C. M., Nonaka, A., Almgren, A. S., Bell, J. B., \& Zingale, M. 2011, apj, 728, 118

Malone, C. M., Zingale, M., Nonaka, A., Almgren, A. S., \& Bell, J. B. 2014, apj, 788, 115

Maraschi, L., \& Cavaliere, A. 1977, in X-ray Binaries and Compact Objects, ed. K. A. van der Hucht, 127-128 
Marsaglia, G., Zaman, A., \& Tsang, W. W. 1990, Statistics \& Probability Letters, 9, 35

Miles, J. W. 1961, Journal of Fluid Mechanics, 10, 496

Pacitto, G., Flament, C., Bacri, J.-C., \& Widom, M. 2000, Phys. Rev. E, 62, 7941

Paczynski, B. 1983, apj, 264, 282

Paczynski, B., \& Proszynski, M. 1986, apj, 302, 519

Press, W. H., Teukolsky, S. A., Vetterling, W. T., \& Flannery, B. P. 1993, Numerical Recipes in FORTRAN; The Art of Scientific Computing, 2nd edn. (New York, NY, USA: Cambridge University Press)

Priedhorsky, W., \& Terrell, J. 1984, apjl, 284, L17

Ramaprabhu, P., \& Andrews, M. J. 2004, Journal of Fluid Mechanics, 502, 233

Rappaport, S., Joss, P. C., \& Webbink, R. F. 1982, apj, 254, 616

Read, K. 1984, Physica D: Nonlinear Phenomena, 12, 45

Ricks, A. J., Hewson, J. C., Kerstein, A. R., et al. 2010, Combustion Science and Technology, 182, 60

Ristorcelli, J. R., \& Clark, T. T. 2004, Journal of Fluid Mechanics, 507, 213

Schatz, H., Aprahamian, A., Barnard, V., et al. 2001, Phys. Rev. Lett., 86, 3471

Shaposhnikov, N., \& Titarchuk, L. 2004, apjl, 606, L57

Spitkovsky, A., Levin, Y., \& Ushomirsky, G. 2002, apj, 566, 1018

Stella, L., Priedhorsky, W., \& White, N. E. 1987, apjl, 312, L17

Strohmayer, T., \& Bildsten, L. 2006, in Cambridge Astrophysics Series, Vol. 39, Compact Stellar X-Ray Sources, ed. W. Lewin \& M. van der Klis (Cambridge, U.K.: Cambridge University Press), 113-156

Strohmayer, T. E., \& Brown, E. F. 2002, apj, 566, 1045 
Sugimoto, D., Ebisuzaki, T., \& Hanawa, T. 1984, pasj, 36, 839

Suzuki, M., Kawai, N., Tamagawa, T., et al. 2007, pasj, 59, 263

Swank, J. H., Becker, R. H., Boldt, E. A., et al. 1977, apjl, 212, L73

Swank, J. H., Becker, R. H., Pravdo, S. H., \& Serlemitsos, P. J. 1976, iaucirc, 2963, 1

Taam, R. E., Woosley, S. E., Weaver, T. A., \& Lamb, D. Q. 1993, pj, 413, 324

Tawara, Y., Hayakawa, S., \& Kii, T. 1984a, pasj, 36, 845

Tawara, Y., Hirano, T., Kii, T., Matsuoka, M., \& Murakami, T. 1984b, pasj, 36, 861

Timmes, F. X. 1999, The Astrophysical Journal Supplement Series, 124, 241

—. 2000, The Astrophysical Journal, 528, 913

- 2015a, 19 isotope network, http://cococubed.asu.edu/code_pages/burn_ helium.shtml, accessed: 2015-09-30

—. 2015b, Helmholtz EOS, http://cococubed.asu.edu/code_pages/eos.shtml, accessed: 2015-09-30

Timmes, F. X., \& Swesty, F. D. 2000, The Astrophysical Journal Supplement Series, 126,501

Turner, N. J., \& Stone, J. M. 2001, apjs, 135, 95

Weaver, T. A., Zimmerman, G. B., \& Woosley, S. E. 1978a, apj, 225, 1021

-. 1978b, apj, 225, 1021

Woosley, S. E., Fowler, W. A., Holmes, J. A., \& Zimmerman, B. A. 1978, Atomic Data and Nuclear Data Tables, 22, 371

Woosley, S. E., \& Taam, R. E. 1976, nat, 263, 101

Woosley, S. E., \& Weaver, T. A. 1984, in American Institute of Physics Conference Series, Vol. 115, American Institute of Physics Conference Series, ed. S. E. Woosley, 273-297 
Woosley, S. E., Heger, A., Cumming, A., et al. 2004, apjs, 151, 75

Woosley, S. E. Fowler, W. A., Holmes, J. A., \& Zimmerman, B. A. 1975, Report (California Institute of Technology) No. OAP-422

Yakovlev, D. G., \& Urpin, V. A. 1980, sovast, 24, 303

Youngs, D. L. 1989, Physica D: Nonlinear Phenomena, 37, 270

Zingale, M., Timmes, F. X., Fryxell, B., et al. 2001, apjs, 133, 195 


\section{Appendix A}

\section{Tests of Random Number Generator}

The random number used in this dissertation is developed by Marsaglia et al. (1990). The algorithm is based on a combination of lagged Fibonacci sequence (with 97 and 33) and an "arithmetic sequence". It has a long period of $2^{144}$ and it can efficiently generate independently disjoint sequences, which is ideal for parallelization computing. The generator has passed stringent tests according to James (1990). Here I present some basic tests of this generator for the purpose of learning testing techniques and checking my implementation.

The tests include two parts: the uniformity test and independence test. For uniformity, a test of covering a $2 \mathrm{D}$ box with random numbers and a $\chi^{2}$ test have been carried out for both one-sequence and two-sequence random numbers. For independence, a correlation test is done for both one-sequence and two-sequence random numbers. Here the two-sequence case is included to test the qualities between different sequences. Two sequences of random numbers with length $n=100,000$ are used in these tests. The seeds are randomly chosen as 12345 and 54321, respectively.

The uniformity test is done by plotting dots with pairs of random numbers as coordinates in a $2 \mathrm{D}$ box. For one-sequence test, the sequence is equally divided into two parts and each part serves as $\mathrm{x}$ or $\mathrm{y}$ coordinates. For two-sequence test, the $\mathrm{x}$ and $\mathrm{y}$ coordinates come from the first halves of the two sequences, respectively. The results 


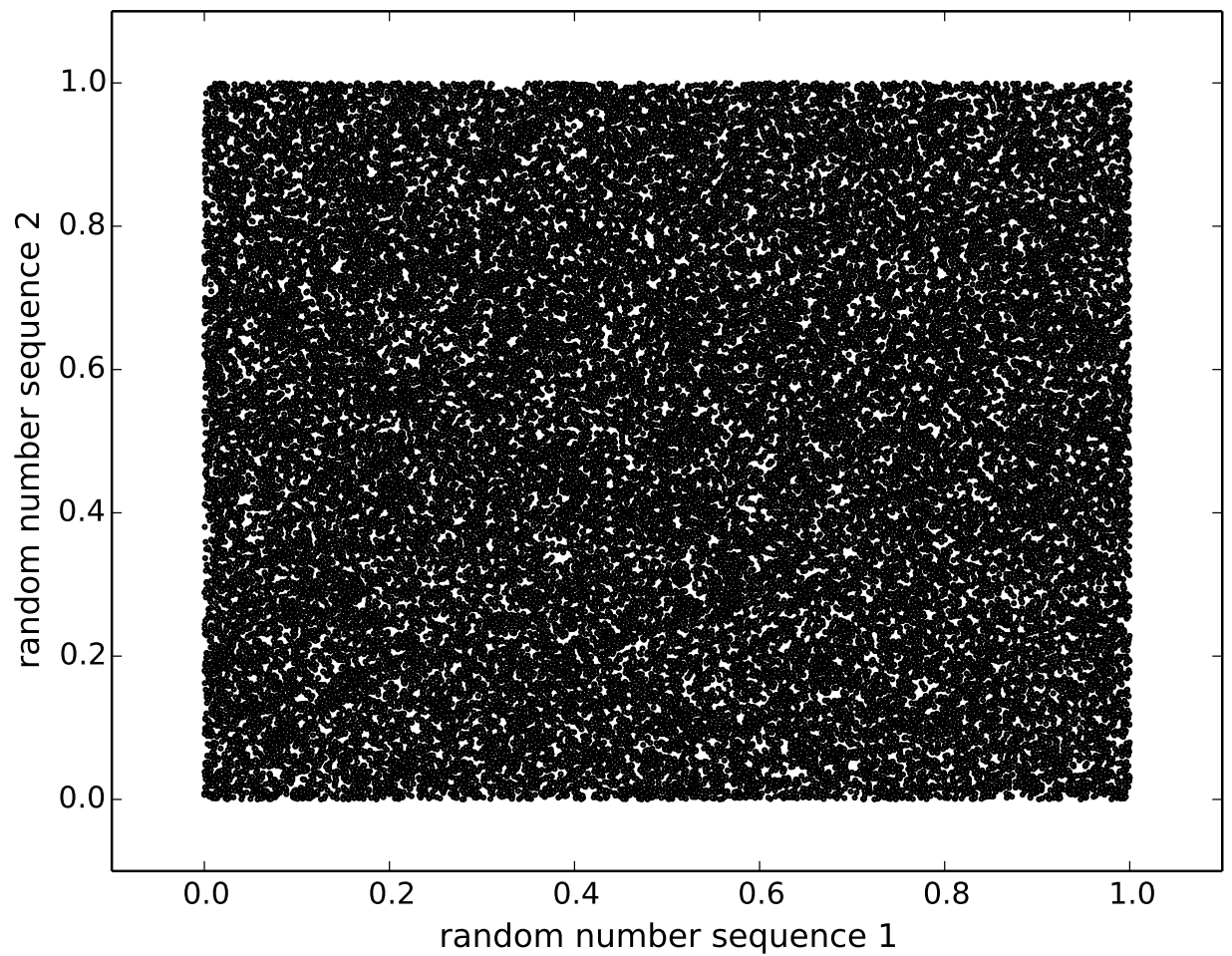

Figure A.1: Uniformity test of one-sequence random numbers. The seed to generate this sequence is randomly chosen as 12345 . It is divided equally into two parts and each part is used as $\mathrm{x}$ or $\mathrm{y}$ coordinates to plot dots in the $2 \mathrm{D}$ box.

are shown in Figures A.1 and A.2. It is clear that the both boxes are covered almost uniformly without any noticeable patterns.

The $\chi^{2}$ test is done by dividing the range $[0,1]$ into $m$ bins and summing the square of deviations from the expected values in all the bins. The formula for $\chi^{2}$ is

$$
\chi^{2}=\frac{\sum_{i=1}^{n=100000}\left(N_{i}-\frac{n}{m}\right)}{\frac{n}{m}},
$$

where $N_{i}$ is the counted number in bin $i$, and $n / m$ is the expected number in bin $i$ for a uniform distribution. Three $m$ values are chosen for this test and the results are summarized in Table A.1. The degree of freedom (DOF) is equal to $m-1$. The random numbers for two-sequence case is obtained in a similar fashion as in the uniformity test. 


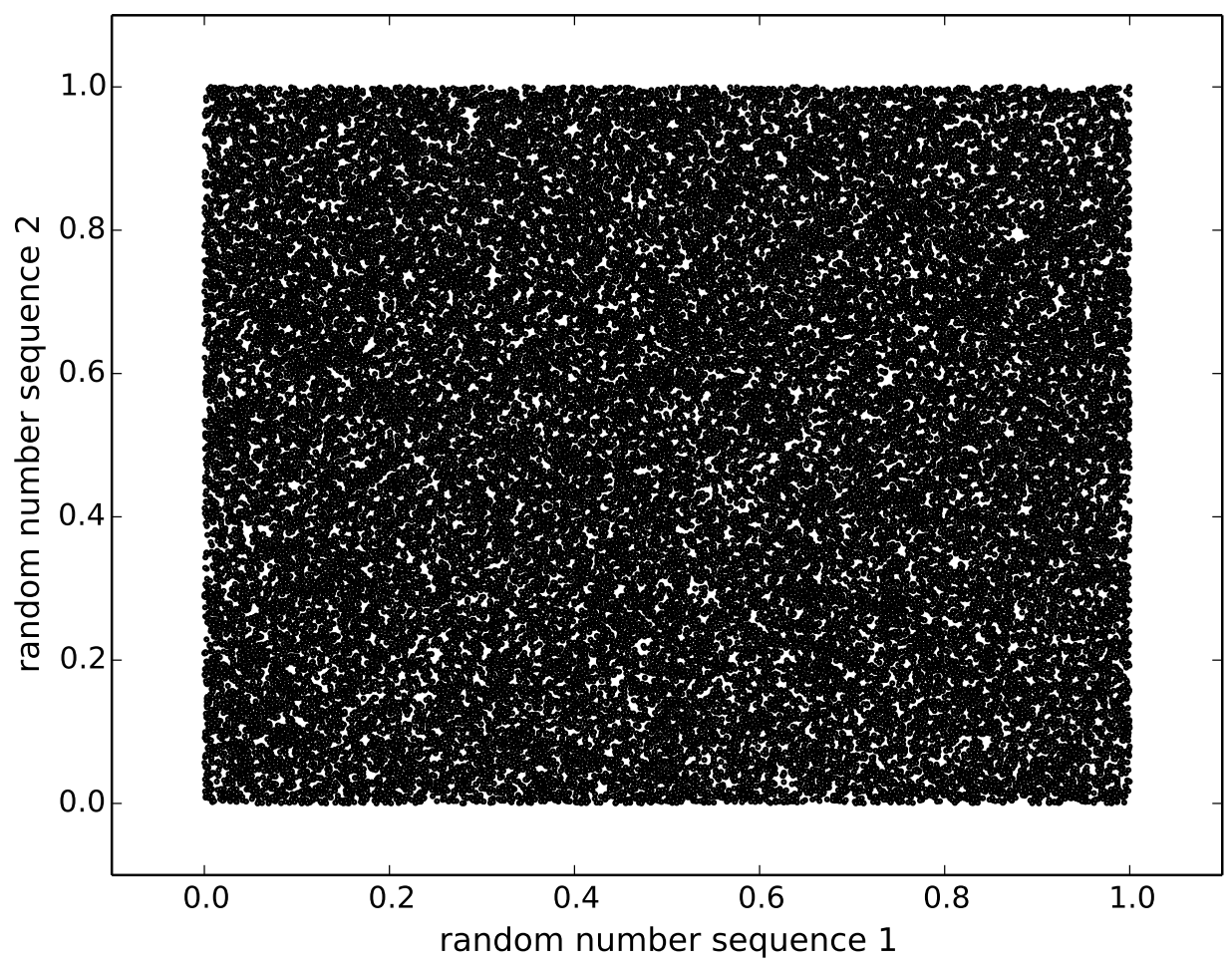

Figure A.2: Uniformity test of two-sequence random numbers. The seeds to generate these two sequences are 12345 and 54321 respectively. The first half of the former is used for $\mathrm{x}$ coordinates while the first half of the latter is used for $\mathrm{y}$ coordinates.

Compared with the $\chi^{2}$ distribution table, most of the $\chi^{2}$ values are in the trusted region (10\%-90\%; Knuth 1997) except the case when DOF $=80$ falling into "suspect" region $\left(95 \%<\chi^{2}<97.5 \%\right)$. Overall, the random number generator passes the $\chi^{2}$ test.

The correlation test is done by calculating correlation factor

$$
\hat{\rho}=\frac{\sum_{i=1}^{n=100000}\left(U_{i}-\bar{U}(n)\right)\left(U_{i+1}-\bar{U}(n)\right)}{\sum_{i=1}^{n=100000}\left(U_{i}-\bar{U}(n)\right)^{2}},
$$

where $U$ represents a random number, and $\bar{U}(n)$ is the sample mean. $U_{n+1}$ is set to $U_{1}$. Three cases are calculated and the results are summarized in Table A.2. The column under "combination" is obtained by using both previous sequences. For $n=100,000$, the probability of $-2 \times 10^{-3} \leq \hat{\rho} \leq 2 \times 10^{-3}$ is about $95 \%$. All three correlation factors 


\begin{tabular}{|c|c|c|c|c|c|c|}
\hline & \multicolumn{3}{|c|}{ One-sequence } & \multicolumn{3}{c|}{ Two-sequence } \\
\hline DOF & 60 & 80 & 100 & 60 & 80 & 100 \\
\hline$\chi^{2}$ & 68.16 & 103.50 & 117.88 & 57.71 & 82.15 & 111.53 \\
\hline
\end{tabular}

Table A.1: $\chi^{2}$ test results

are within this range, so the correlation test has passed.

\begin{tabular}{|c|c|c|c|}
\hline & Seed=12345 & Seed=54321 & Combination \\
\hline$\hat{\rho}$ & $1.90 \times 10^{-4}$ & $-6.66 \times 10^{-4}$ & $1.10 \times 10^{-3}$ \\
\hline
\end{tabular}

Table A.2: Correlation test results.

In conclusion, the random number generator has passed both tests and it produces independently disjoint sequence with good quality and fast speed. 